$\mathrm{UCD}-95-18$

May, 1995

\title{
A MINIMAL FOUR-FAMILY SUPERGRAVITY MODEL
}

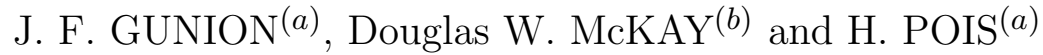 \\ (a) Davis Institute for High Energy Physics, \\ Department of Physics, U. C. Davis, Davis, CA 95616 \\ (b) University of Kansas, Department of Physics and Astronomy, \\ Lawrence, Kansas, 66045
}

\begin{abstract}
In this work, we investigate the phenomenology of minimal four-family MSSM supergravity theories containing an additional generation $\left(t^{\prime}, b^{\prime}, \tau^{\prime}, \nu^{\prime}\right)$ of heavy fermions along with their superpartners. We constrain the models by demanding: gauge coupling constant unification at high energy scales; perturbative values for all Yukawa couplings for energy scales up to the grand-unification scale; radiative electroweak (EW) symmetry breaking via renormalization group evolution down from the grand-unification scale; a neutral LSP; and consistency with constraints from direct searches for new particles and precision electroweak data. The perturbative constraints imply a rather light fourth-family quark and lepton spectrum, and $\tan \beta \lesssim 3$. The lightest CP-even Higgs mass receives fourth-family loop corrections that can result in as much as a $30 \%$ increase over the corresponding three-family mass value. Significant fourth-family Yukawa coupling contributions to the evolution of scalar masses lead to unexpected mass hierarchies among the sparticles. For example, the $\widetilde{\tau}_{1}^{\prime}$ is generally the lightest slepton and the lightest squark is the $\widetilde{b}_{1}^{\prime}$. A significant lower bound is placed on the gluino mass by the simple requirement that the $\widetilde{\tau}_{1}^{\prime}$ not be the LSP. Sleptons of the first two families are much more massive compared to the LSP and other neutralinos and charginos than in the three-family models; in particular, all sleptons belonging to the first three families could easily lie beyond the reach of a $\sqrt{s}=500 \mathrm{GeV} e^{+} e^{-}$collider. Consistency tests of the RGE equations via mass sum rules and relations are explored. Relations between slepton masses and gaugino masses are shown to be very sensitive to the presence of a fourth generation. The most important nearfuture experimental probes of the four-family models are reviewed. A scenario with $m_{t} \sim m_{W}$ and $t \rightarrow \widetilde{t}_{1} \widetilde{\chi}_{1}^{0}$ is shown to be inconsistent with universal softSUSY-breaking boundary conditions. Full four-family evolution of $\alpha_{s}$ is shown to lead to a significant enhancement in inclusive jet and di-jet spectra at Tevatron energies when all sparticle masses are near their lower bounds.
\end{abstract}

\section{Introduction}

Despite the success of the Standard Model (SM), it is almost certainly incomplete. In particular, the full theory should include a quantum theory of gravity. However, the huge discrepancy between the characteristic Planck scale $\left(M_{P l} \simeq 10^{18} \mathrm{GeV}\right)$ and the weak scale, $\mathcal{O}\left(m_{Z}\right)$, is not easily bridged without encountering problems related to fine-tuning and gauge-hierarchy. Supersymmetry is currently the only fully quantitative and consistent resolution to these problems. In 
particular, the Minimal Supersymmetric extension of the Standard Model (MSSM) is an extremely attractive contender for physics beyond the SM due to its natural prediction that the gauge couplings all unify at a common grand-unification (GUT) scale, $M_{U}$. The precise particle content of the MSSM (comprising sparticle partners for all SM particles and exactly two Higgs doublet fields $H_{1}, H_{2}$ and their higgsino partners) is crucial to this success. In addition, the MSSM provides an attractive explanation for the long proton decay lifetime and an attractive candidate (the lightest supersymmetric particle, or LSP) for cold dark matter, easily accommodates the absence of significant flavor-changing neutral currents (FCNC's), and is completely consistent with current precision electroweak data. Of course, experiment has yet to reveal any evidence for SUSY, in either the sparticle or the Higgs sector.

Unfortunately, supersymmetric models in general, and the MSSM in particular, provide no explanation for the number of families (we denote the number of families/generations by $N_{g}$ ) or the patterns of fermion masses. Certainly, a supersymmetric extension of the standard three-family model is entirely consistent, but the possibility of incorporating one or more additional generations into the MSSM framework deserves exploration. In a previous work, ${ }^{[1]}$ we investigated the minimal four-family, gauge-unified MSSM, with a large Dirac mass for the $\nu^{\prime{ }^{[2]}}$ so as to be consistent with LEP constraints. ${ }^{[3]}$

In particular, we explored the constraints upon the model obtained by generalizing the usual perturbative limit on the top quark Yukawa coupling $\lambda_{t}$ to the requirement that all Yukawa couplings remain perturbative at energy scales below $M_{U}$. Assuming that the CDF and D0 'top-quark' events arise from a third generation top with $m_{t} \gtrsim 155 \mathrm{GeV}^{\star}$, we demonstrated that perturbative Yukawa behavior requires a rather light fourth-family spectrum, namely $m_{t^{\prime}, b^{\prime}} \lesssim m_{t}$. The fourthfamily lepton sector is even more strongly constrained: $m_{\tau^{\prime}, \nu^{\prime}} \lesssim 85 \mathrm{GeV}$, the upper limit occurring when $m_{t^{\prime}}, m_{b^{\prime}}$ are just beyond the reach of LEP. (That is, the upper limits for the fourth-family leptons versus quarks have a strong inverse correlation.) Additional results included: i) limits requiring $\tan \beta\left(\tan \beta \equiv v_{2} / v_{1}, v_{1}\right.$ and $v_{2}$ being the vacuum expectation values of the $H_{1}$ and $H_{2}$ scalar fields) to have a very modest value, roughly $1 \lesssim \tan \beta \lesssim 3$, in order to avoid perturbativity problems for the Yukawas; ii) the impossibility of imposing the $\lambda_{b}\left(M_{U}\right) / \lambda_{\tau}\left(M_{U}\right)=1$ boundary condition in four-family models; and iii) an increase in the predicted value for $\alpha_{3}\left(m_{Z}\right)$ by $\simeq+3 \%$ from the purely gauge coupling contributions to gauge coupling running, which could, however, be largely compensated by two-loop Yukawa contributions to gauge coupling running. ${ }^{[1]}$

In this paper we elaborate upon our earlier results and extend our four family study to include the superpartners of the fourth-family quarks and leptons. We adopt the conventional framework in which the MSSM parameters are determined in the context of minimal supergravity by universal (at $M_{U}$ ) soft-SUSY-breaking

$\star$ We define the fourth generation by the CKM matrix hierarchy $\left|V_{t b}\right|^{2},\left|V_{t^{\prime} b^{\prime}}\right|^{2} \sim 1$, with $\left|V_{t^{\prime} b}\right|^{2},\left|V_{t b^{\prime}}\right|^{2} \ll 1$. 
masses for the gauginos $\left(m_{1 / 2}\right)$ and for the squark and Higgs boson fields $\left(m_{0}\right)$, and universal soft Yukawa couplings $(A)$; of course, these typically evolve to (rather disparate) weak-scale values. Additional crucial parameters are: $\tan \beta$ (defined above) and $\mu$, the coefficient of the $\hat{H}_{1} \hat{H}_{2}$ mixing term in the superpotential. Aside from having considerable theoretical motivation, this approach has the advantage that relatively few independent parameters, $\tan \beta, m_{t}, m_{0}, A, m_{1 / 2}$ (along with the sign of $\mu$ ), are sufficient to completely specify the theory at the weak scale. ${ }^{\dagger}$

Specific supergravity (SUGRA) and string models make definite predictions for the relative sizes of $m_{0}, A$ and $m_{1 / 2}$. It is convenient to specify a given model in terms of the ratios $\xi_{A}=A / m_{1 / 2}$ and $\xi_{0}=m_{0} / m_{1 / 2}$. Two models provide particularly useful benchmarks. The first is the string-motivated dilaton boundary condition scenario specified by $\xi_{0}=1 / \sqrt{3}, \xi_{A}=-1$. This boundary condition set emerges universally in all string models where supersymmetry breaking is dilaton dominated. It represents a middle-of-the-road choice in that the gaugino mass $m_{1 / 2}$ and the soft scalar mass $m_{0}$ are both of importance in the final low-energy values of the squark and slepton masses, but sleptons are generally significantly lighter than squarks, and both are generally lighter than the gluino. A more extreme boundary condition choice is the 'no-scale' model with $\xi_{0}=\xi_{A}=0$, which can also arise in certain string and supergravity approaches. In this model, supersymmetry breaking arises entirely from the gaugino mass $m_{1 / 2}$ at $M_{U}$, with all other supersymmetry breaking parameters generated by RGE evolution as the energy scale decreases. Sleptons are still lighter compared to squarks, and the gluino mass is generally the largest. A brief review of these two boundary conditions can be found in Ref. [5]. As a final benchmark possibility we shall also consider $\xi_{0}=-\xi_{A}=1$. In this case, the slepton and squark masses turn out to be large (often larger than the gluino mass) and fairly similar in size due to the dominance of the $m_{0}$ source term.

Our study is designed to complement the existing MSSM and supergravity (SUGRA) studies, virtually all of which have assumed three generations, ${ }^{[6]}$ and the many earlier studies of a four-family SM. ${ }^{[7]}$ Since the LEP experiments rule out the possibility of an additional new sequential 'light' neutrino, ${ }^{[8]}$ the fourth-family neutrino must be quite massive, $m_{\nu^{\prime}}>45 \mathrm{GeV}$, and the fourth family would seem to not be truly 'sequential'. However, there are many indications of small non-zero mass for the neutrinos of the first three families, in which case the much larger mass for the $\nu^{\prime}$ is no different than the large value of the top quark mass as compared to the masses of the other quarks. Both of these large generational hierarchies must find explanation in physics beyond the MSSM. A fourth family with a heavy neutrino is no more unnatural than a third family with a heavy top quark. ${ }^{[9]}$

In the minimal supersymmetric model with $N_{g} \leq 4$, the gauge couplings unify $^{[10]}$ perturbatively at a common scale $M_{U} \simeq 2-5 \times 10^{16} \mathrm{GeV}$. However, as noted earlier, for $N_{g}=4$ we must relax the often-imposed theoretical prejudice

\footnotetext{
$\dagger$ For a review of this approach, see Ref. [4] and references therein.
} 
that $\lambda_{b}\left(M_{U}\right)=\lambda_{\tau}\left(M_{U}\right)$ if we are to generate an acceptable prediction for $m_{b} / m_{\tau}$ at low energies. We do not regard this as a significant difficulty since there are many reasons why the Yukawa couplings might not be unified at $M_{U}$. In addition to potentially large weak and GUT-scale threshold effects, ${ }^{[11]}$ a different field content can drastically modify the GUT-scale relations among the Yukawa couplings. For example, if a 45-Higgs is added to the $S U(5)$ GUT theory, then the strict relation $\lambda_{b}\left(M_{U}\right) / \lambda_{\tau}\left(M_{U}\right)=1$ is no longer valid. ${ }^{[12]}$ More recently, the authors in Ref. [13] have shown that if the theory contains additional heavy fermions at $M_{U}$ then $\lambda_{b}\left(M_{U}\right) / \lambda_{\tau}\left(M_{U}\right)<1$ can naturally result.

Thus, it is of considerable interest to study the phenomenology of a four-family MSSM model. In our study, we will delineate how current experiments can either eliminate or confirm the existence of a fourth family. We shall also discuss a number of theoretical subtleties that arise in the implementation of four generations in the context of the SUGRA framework. Aside from updating the constraints on $m_{t^{\prime}}$, $m_{b^{\prime}}$, and $\tan \beta$, we will particularly address the following specific issues. i) When all basic constraints are applied, what are the experimentally allowed regions of parameter space? ii) Does the electroweak (EW) radiative breaking mechanism work in the $N_{g}=4$ case? iii) What are the additional radiative corrections to the lightest SUSY Higgs mass? iv) How is the general spectrum of the sparticles affected by the presence of four families? v) In particular, are there new constraints on $M_{S U S Y}$ arising from fourth-family sparticle mass constraints? vi) More generally, how does the fourth family sparticle spectrum compare to the spectra of the first three families? And, vii) what experimental constraints are imposed by the latest $D 0$ and CDF top quark searches, as well as a global fit to the latest EW precision data? We examine each of these issues, although not precisely in the above order.

Section 2 is devoted to a study of gauge and Yukawa coupling unification. In particular, we refine our earlier analysis to include exact (numerical) solution of the coupled two-loop gauge and Yukawa couplings. The parameter space regions given by demanding perturbativity for the Yukawa couplings and consistency with nonobservation of sparticles and fourth-family fermions at LEP are specified. Section 3 discusses the radiative electroweak symmetry breaking mechanism. Section 4 describes the physical Higgs boson masses and their phenomenology in the fourgeneration MSSM. Section 5 discusses a constraint on the SUSY sparticle mass scales that is peculiar to the four-generation MSSM model. Section 6 presents, for a number of typical models, the sparticle mass spectra that arise from a four-family scenario, and delineates allowed regions of soft-supersymmetry-breaking parameter space after all direct experimental constraints have been imposed. Key modelindependent features of the sparticle spectra are identified. Section 7 reviews the latest direct collider limits on a fourth family, including the implications of the latest D0 and CDF data. There, we demonstrate that a scenario ${ }^{[14]}$ in which the top is light, $m_{t} \sim m_{W}$, but not observed because it decays by the mode $t \rightarrow \widetilde{t}_{1} \widetilde{\chi}_{1}^{0}$ (the CDF/D0 events coming from $t^{\prime} \rightarrow b W$ in this scenario), is not consistent with universal soft-SUSY-breaking boundary conditions by virtue of the Section 5 constraint which forces the $\widetilde{t}_{1}$ to be very heavy. In addition, a global fit to the latest precision LEP data is presented and the ensuing constraints on a fourth family are 
discussed. We also explore implications of a fourth family for the inclusive-jet and di-jet spectra of the light quarks following from the dramatic slow-down in the evolution of $\alpha_{s}$ once energy scales above $M_{S U S Y}$ are reached. Section 8 presents our summary and concluding remarks. The renormalization group formulae and beta functions that are frequently referenced in the text appear in Appendix A. The question of the accuracy with which $\tan \beta$ can be determined by squark/slepton mass measurements is discussed in Appendix B.

\section{Gauge and Yukawa Coupling Unification for $\mathrm{N}_{\mathrm{g}}=4$}

Gauge coupling unification and the low energy prediction for $m_{b} / m_{\tau}$ (assuming $\lambda_{b}\left(M_{U}\right)=\lambda_{\tau}\left(M_{U}\right)$ ) at the two-loop level in an MSSM four-family model were first considered in Ref. [15]. For complete family representations, gauge coupling unification implies values for $\alpha_{3}\left(m_{Z}\right)$ and $M_{U}$ that are independent of the number of families at the one-loop level. At two-loops, there is a weak dependence on $N_{g}$. Fig. 1 shows the unification of the couplings in both the three- and fourfamily scenarios including two-loop gauge contributions to the the beta functions but not including the two-loop Yukawa contributions. Assuming the published value of $\alpha_{e m}^{-1}\left(m_{Z}\right)=127.9$ (in the $\overline{M S}$ scheme), and adopting $\sin ^{2} \theta_{W}\left(m_{Z}\right)=$ 0.2316 (see the discussion of the next paragraph), and a single SUSY breaking scale $M_{S U S Y}=m_{Z}{ }^{\star}$, we predict $\alpha_{3}^{0}\left(m_{Z}\right)=(0.1283,0.1326), \alpha_{U}^{0}=(0.0432,0.0917)$ and $M_{U}^{0}=(2.98,5.73) \times 10^{16} \mathrm{GeV}$ for $N_{g}=(3,4)$, respectively. Here, the superscript ' 0 ' indicates that the two-loop Yukawa effects have not been included. We see that there is a slight shift in $\alpha_{3}\left(m_{Z}\right)$, a significant shift in $M_{U}$, and a factor of two increase in $\alpha_{U}^{0}$ as one moves from $N_{g}=3$ to $N_{g}=4$. For the more recent value ${ }^{[17]}$ of $\alpha_{e m}^{-1}\left(m_{Z}\right)=129.08$ (= 128.05 in the $\overline{M S}$ scheme), and adjusting $\sin ^{2} \theta_{W}\left(m_{Z}\right)$ downwards by $-0.0002,{ }^{[16]}$ to $\sin ^{2} \theta_{W}\left(m_{Z}\right)=0.2314$, the resulting predictions are $\alpha_{3}^{0}\left(m_{Z}\right)=(0.129,0.133), \alpha_{U}^{0}=(0.0432,0.092)$ and $M_{U}^{0}=(3.25,6.28) \times 10^{16} \mathrm{GeV}$ for $N_{g}=(3,4)$. In either case, inclusion of a fourth family raises $\alpha_{3}\left(m_{Z}\right)$ by about $3 \%$ when calculated at two-loop order without including Yukawa contributions to the two-loop beta functions. We shall shortly return to this issue.

In all the calculations that follow, we shall employ the published value for the electromagnetic coupling of $\alpha_{e m}^{-1}\left(m_{Z}\right)=127.9$ in the $\overline{M S}$ scheme and keep the $\overline{M S}$ value of $\sin ^{2} \theta_{W}\left(m_{Z}\right)$ fixed at $\sin ^{2} \theta_{W}\left(m_{Z}\right)=0.2316$. Holding $\sin ^{2} \theta_{W}(m z)$ fixed is an approximation. The actual 'best-fit' value of $\sin ^{2} \theta_{W}\left(m_{Z}\right)$ for a given set of experimental data depends upon the top-quark mass, the masses of the fourth-family fermions, the mass of the light SM-like Higgs boson of the model, and the masses of the superpartners of all four families (see, for example, Refs. [16,18]). If exact coupling constant unification is demanded, and definite boundary conditions for the soft-supersymmetry-breaking parameters are specified at the unification scale,

* As described in Ref. [16], the effective $M_{S U S Y}$ is generally not far from $m_{Z}$ even when some superpartners are heavy. 
the appropriate 'best-fit' value for $\sin ^{2} \theta_{W}\left(m_{Z}\right)$ value within the four-generation model could be obtained by a self-consistent iterative procedure such as described in Refs. [16,18]. We have chosen to avoid this complexity for this first study of the four-generation supersymmetric model. We also note that we ignore the differences between $\overline{D R}$ and $\overline{M S}$ couplings and masses. In particular, although it is the $\overline{D R}$ couplings that are most naturally required to unify in supersymmetric models, the differences between $\overline{D R}$ and $\overline{M S}$ couplings are not significant at scale $M_{S U S Y} \sim m_{Z}$ compared to other uncertainties. As described later, we allow a certain level of 'error' in the unification of the coupling constants in order to account for the remaining experimental uncertainty in $\sin ^{2} \theta_{W}\left(m_{Z}\right)$, the slight $\overline{D R}$ vs. $\overline{M S}$ mismatch and the (small) variation in $\sin ^{2} \theta_{W}\left(m_{Z}\right)$ that would occur as we change 4th-family masses and SUSY parameters. We will see that the allowed parameter space is only slightly sensitive to a relaxation of exact unification, and certainly the general phenomenological features and issues that we discuss would not be significantly altered by a more precise treatment.

The $m_{b^{\prime}}, m_{t^{\prime}}$ parameter space for $m_{\nu^{\prime}}=m_{\tau^{\prime}}=50 \mathrm{GeV}$ (consistent with LEP limits of $45 \mathrm{GeV}^{[3]}$ ) and $m_{t}=165 \mathrm{GeV}$, equivalent to $m_{t}($ pole $)=175 \mathrm{GeV}^{\dagger}$, that is allowed after demanding perturbativity for the Yukawa couplings for all energy scales up to $M_{U}$, as well as coupling constant unification at $M_{U}$, including two-loop Yukawa contributions to the gauge coupling evolution equations, is illustrated for $\tan \beta=1.5$ and $\tan \beta=2.2$ in Fig. 2. Our precise criterion for perturbativity is that all Yukawa couplings obey $\lambda_{i} \leq 3.3$; this value ensures that one-loop effects dominate over two-loop effects. ${ }^{[19]}$ Two possible levels of gauge unification are considered: unification of $\alpha_{3}$ with $\alpha_{1}$ and $\alpha_{2}$ to within $2.5 \%$ and to within $0.01 \%$. Fig. 2 shows the allowed parameter space regions for these two cases. We observe that some of the excluded points on the border become allowed if the precision demanded for unification of the couplings is relaxed by even a few percent. Our procedure and these results will be explained in more detail shortly.

The allowed parameter space regions illustrated in Fig. 2 are even more restricted than those given in our earlier work, Ref. [1], at these same $\tan \beta$ values, for two reasons: i) the higher value of $m_{t}\left(m_{t}\right)=165 \mathrm{GeV}\left(v s . m_{t}\left(m_{t}\right)=160 \mathrm{GeV}\right)$ has been chosen so as to yield $m_{t}($ pole $) \sim 175 \mathrm{GeV}$, consistent with the latest CDF and D0 experimental results ${ }^{[20]}$ (the precise pole mass value depends on the sparticle spectrum, but only weakly); ii) the two-loop Yukawa effects in the running of the gauge couplings feed back into the Yukawa couplings themselves so that they violate perturbativity more easily. This latter point can be understood by noting that inclusion of Yukawa couplings reduces the value of $\alpha_{3}(Q)$ when $m_{t^{\prime}}, m_{b^{\prime}}$ have values near the boundary of the perturbatively allowed region. The reduction in $\alpha_{3}(Q)$ in turn slightly reduces the magnitude of the negative gauge contribution to the one-loop component of the Yukawa beta functions as $Q$ runs from $m_{Z}$ to $M_{U}$ (see Eqs. (A.1), (A.5) and (A.6) in Appendix A), constraining the weak-scale value

$\dagger$ Unless explicitly indicated, up until Section 7 all masses are the running $m(m)$ masses, and not the pole masses. 
of the Yukawas to be somewhat smaller than before in order to remain perturbative at $M_{U}$.

This reduction in $\alpha_{3}$ for points at the parameter space edge is illustrated in Fig. 3 which shows how much the values of $M_{U}$ and $\alpha_{3}\left(m_{Z}\right)$ can be affected by including the large Yukawa contributions to the two-loop gauge coupling beta functions in the four-family scenario. The values of $M_{U}$ and $\alpha_{3}\left(m_{Z}\right)$ are presented as contours in the the $m_{t^{\prime}}, m_{b^{\prime}}$ plane. The shifts in $\alpha_{3}\left(m_{Z}\right), M_{U}$ from $\alpha_{3}^{0}\left(m_{Z}\right), M_{U}^{0}$ agree well with our approximate solutions from Ref. [1]. For $m_{t^{\prime}}, m_{b^{\prime}}$ near their maximal allowed values, $\alpha_{3}\left(m_{Z}\right), M_{U}$ can be lowered by as much as $5 \%, 16 \%$ respectively. We see that the $N_{g}=4$ values of $\alpha_{3}\left(m_{Z}\right)$ are essentially the same as the $N_{g}=3$ value when large Yukawa effects are included at two-loop.

The precise boundary of the perturbatively allowed region depends upon the precision demanded for coupling constant unification. In Fig. 2 we showed the allowed regions obtained by demanding unification of the coupling constants to within $2.5 \%$ or $0.01 \%$ (the latter being essentially equivalent to exact unification). The allowed region in the $0.01 \%$ case is slightly reduced compared to the $2.5 \%$ case. This sensitivity is implicitly present even before the two-loop Yukawa terms are included in gauge coupling evolution. As noted above, by increasing $\alpha_{3}$ the Yukawa blow-up is delayed. So if $\alpha_{3}\left(M_{U}\right)$ is allowed to be slightly larger than the common value of $\alpha_{1}\left(M_{U}\right)$ and $\alpha_{2}\left(M_{U}\right)$ (denoted $\alpha_{1,2}\left(M_{U}\right)$ ), then slightly larger values of $m_{t^{\prime}}, m_{b^{\prime}}$ will be allowed by the requirement of perturbativity for Yukawa couplings up to $M_{U}$. When two-loop Yukawa couplings are included in the gauge evolution equations, an iterative procedure must be employed for finding a fully consistent solution. For a given $m_{t^{\prime}}, m_{b^{\prime}}$ choice and a given possible starting value of $\alpha_{3}\left(m_{Z}\right)$, full two-loop evolution of all three couplings may be performed (which requires evolving also the Yukawas at the same time). The value of $M_{U}$ at which $\alpha_{1}$ and $\alpha_{2}$ unify can then be determined. The common value, $\alpha_{1,2}\left(M_{U}\right)$, can then be compared to the evolved $\alpha_{3}\left(M_{U}\right)$ value. This process is iterated until the evolved $\alpha_{3}\left(M_{U}\right)$ value is as close to $\alpha_{1,2}\left(M_{U}\right)$ as possible without the Yukawas becoming too large $\left(\lambda_{i}<3.3\right.$ is required). The precision of unification is then specified by the requirement that $\alpha_{3}\left(M_{U}\right)=\alpha_{1,2}\left(M_{U}\right)$ to within a definite percentage deviation: for example unification to within $2.5 \%$ means that $\alpha_{3}\left(M_{U}\right)$ is allowed to be no more than $2.5 \%$ larger than $\alpha_{1,2}\left(M_{U}\right)$. Given experimental errors in $\sin ^{2} \theta_{W}\left(m_{Z}\right)$ and variations in its best fit value as fourth-generation fermion masses and sparticle masses for all the generations are varied, we regard the region allowed by unification to within $2.5 \%$ as fully acceptable. However, to go much beyond the $2.5 \%$ allowed region of Fig. 2 would almost certainly require accepting the fact that one of the Yukawa couplings becomes non-perturbative at a scale below $M_{U}$ or that $\alpha_{3}\left(M_{U}\right)$ truly exceeds $\alpha_{1,2}\left(M_{U}\right)$, e.g. due to non-renormalizable operators, string threshold effects and/or evolution between $M_{U}$ and $M_{\text {string }}$.

$\ddagger$ Not shown is $\alpha_{U}$; the shift in $\alpha_{U}$ due to inclusion of two-loop Yukawa contributions is comparable to the $\alpha_{3}\left(m_{Z}\right)$ shift, i.e. $\lesssim 4 \%$. 
Of course, as at one loop, the value of $\alpha_{3}\left(m_{Z}\right)$ consistent with unification is sensitive to the effective scale $M_{S U S Y}$ implied by the sparticle masses. As the value for $M_{S U S Y}$ is raised, $\alpha_{3}\left(m_{Z}\right)$ is reduced; for $N_{g}=3$ and $M_{S U S Y}=1 \mathrm{TeV}$, the shift can be as large as $\simeq-10 \%$. Similar results hold for $N_{g}=4$ as well. Given the present measurement of $\alpha_{3}\left(m_{Z}\right)=0.12 \pm 0.01$, it would seem that both the $N_{g}=3$ and $N_{g}=4$ scenarios predict an $\alpha_{3}\left(m_{Z}\right)$ which is somewhat high unless $M_{S U S Y}$ is significantly above $m_{Z}$. However, a large value of the effective $M_{S U S Y}$ is not easy to achieve, ${ }^{[16]}$ when the slepton, neutralino and chargino mass scales are significantly lower than the squark and gluino mass scales. Nonetheless, it is fair to say that $\alpha_{3}\left(m_{Z}\right)$ per se does not discriminate between the $N_{g}=3$ and $N_{g}=4$ cases, since the increase in $\alpha_{3}\left(m_{Z}\right)$ for $N_{g}=4$ vs. 3 from pure gauge effects can be compensated in the $N_{g}=4$ case by the large Yukawa effects in the gauge running, as just discussed. In addition, we shall find that $N_{g}=4$ scenarios characteristically force all the sleptons and squarks to higher masses (including those of the first three families), implying that the effective $M_{S U S Y}$ could be larger for $N_{g}=4$ than for $N_{g}=3$. Given this and the additional uncertainties associated with weak and GUT-scale threshold effects, we believe that the $N_{g}=3$ and $N_{g}=4$ scenarios are equally admissible. Attempts to address the moderately high prediction for $\alpha_{3}\left(m_{Z}\right)$ (that emerges in both cases) have recently been considered in Refs. [16,18].

In general, it is important to note that two-loop contributions to the running of $\alpha_{s}(Q)$ will be much more significant in the case of $N_{g}=4$ than for $N_{g}=3$. This is simply due to the fact that the one-loop beta function for $\alpha_{s}$ is proportional to $\left(9-2 N_{g}\right)$ (once $Q>M_{S U S Y}$ ) and therefore is rather small for $N_{g}=4$. This means that two-loop contributions can represent a much larger percentage of the total beta function than in the $N_{g}=3$ case. The importance of two-loop contributions will be especially apparent when considering the running of the squark masses, where some terms involve the running value of $\alpha_{s}^{3}$. The cumulative effect of two-loop contributions can be large in such cases when evolving all the way down from $Q=M_{U}$ to $Q=m_{Z}$. The situation for $\alpha_{1}$ and $\alpha_{2}$ is quite the opposite. Indeed, the one-loop beta function for $\alpha_{2}$ increases by a factor of 3 in going from $N_{g}=3$ to $N_{g}=4$, implying that $\alpha_{2}$ increases much more rapidly as the energy scale is varied from $m_{Z}$ up to $M_{U}$.

What about Yukawa coupling constant unification? In Fig. 4, contours of constant $\lambda_{b^{\prime}}\left(M_{U}\right), \lambda_{t^{\prime}}\left(M_{U}\right)$ and $\lambda_{\tau^{\prime}}\left(M_{U}\right)$ within the allowed parameter space region are shown. From these contours we discover a number of important facts. First, $\lambda_{b^{\prime}}\left(M_{U}\right)$ and $\lambda_{\tau^{\prime}}\left(M_{U}\right)$ are generally quite different; Yukawa unification does not generally occur, although we see that in the $m_{t^{\prime}}, m_{b^{\prime}} \sim 100 \mathrm{GeV}$ corner of the $\tan \beta=1.5$ plot we do have $\lambda_{t^{\prime}} \sim \lambda_{b^{\prime}} \sim \lambda_{\tau^{\prime}}$. Second, we see that most of the non-perturbative borders are defined by one of the fourth-family Yukawa couplings becoming non-perturbative. At $\tan \beta=1.5$ the right-hand border results from nonperturbative behavior for $\lambda_{b^{\prime}}$ (at the upper boundary, $\lambda_{t}$ becomes non-perturbative) while at $\tan \beta=2.2$ the right-hand border results when $\lambda_{\tau^{\prime}}$ becomes large and the upper boundary arises from non-perturbative behavior of $\lambda_{t^{\prime}}$. However, it is also clear that, in general, not all of the fourth-family Yukawas (or $\lambda_{t}$ ) are simultaneously large. This means that one is unlikely to be particularly close to a 
Yukawa fixed-point solution ${ }^{[21]}$ such that the low-energy values of the $\lambda$ 's are rather independent of their $M_{U}$-scale values. The nearest approach to a fixed point occurs in the $m_{t^{\prime}} \sim m_{b^{\prime}} \sim 100 \mathrm{GeV}$ corner of the $\tan \beta=1.5$ figure; the actual fixed point location is indicated by an $X$. In our earlier work, ${ }^{[1]}$ with $m_{t}\left(m_{t}\right)=160 \mathrm{GeV}$ and two-loop Yukawa contributions to gauge running not included in determining the parameter space, the large $m_{t^{\prime}}, m_{b^{\prime}}$ corner of the allowed region was nearer to this fixed point.

\section{EW Symmetry Breaking}

One of the many nice features of the MSSM extended by minimal SUGRA is that, for a very large region of soft-SUSY-breaking parameter space, radiative EWsymmetry breaking is automatically induced by renormalization group evolution. Thus, the hierarchy between $M_{U}$ (where scalar masses are universal and (therefore) EW symmetry is initially unbroken) and $m_{Z}$ receives a natural explanation. In the three-family model, this EW-symmetry breaking is mainly a result of the quantum corrections arising from the large $\lambda_{t}$ Yukawa coupling which drives the $H_{2}$-Higgs field squared-mass to ever smaller values as the energy scale is decreased; EWSB occurs when the Higgs mass-squared term in the scalar Higgs potential is finally driven to a negative value. Of course, to obtain the precise value of $m_{Z}$ as given by $m_{Z}^{2}=\frac{1}{2}\left(g^{2}+g^{\prime 2}\right)\left(v_{1}^{2}+v_{2}^{2}\right)$, there must be a relation among the supersymmetric model parameters. Mathematically, these relations result from requiring that the first derivatives of the scalar field potential with respect to $v_{1}$ and $v_{2}$ vanish. Normally, one of these conditions is used to determine the magnitude (but not the sign) of $\mu$ in terms of the other initial parameters of the theory, including the soft-SUSYbreaking parameters. The other condition is used to determine the magnitude of $m_{3}^{2}$, appearing in the $-m_{3}^{2}\left(H_{1} H_{2}+\right.$ h.c.) mixing term in the scalar field potential. Thus, $\mu$ and $m_{3}^{2}$ at the weak scale become functions of $m_{t^{\prime}}, m_{b^{\prime}}, \tan \beta, m_{Z}, m_{0}$, $m_{1 / 2}$ and $A$. Since this radiative breaking mechanism is essential to the viability of the MSSM plus minimal SUGRA, we describe its dynamics for $N_{g}=4$ before turning to predictions for the SUSY sparticle spectra, and experimental constraints from direct particle searches and EW precision measurements. Not surprisingly, we find that in a four-family scenario the additional large Yukawa couplings also feed into the running of the Higgs field squared-masses, and modify the running significantly. To indicate the way in which radiative breaking occurs in the four-family case, it is convenient to present the discussion at tree-level. A tree-level discussion is adequate for general understanding, and is a good approximation so long as the tree-level minima obey all of the necessary stability and consistency constraints. ${ }^{[22]}$ However, we emphasize that our full numerical calculations are actually performed by minimizing the full scalar potential at one-loop.

Figures 5a and 5b illustrate how radiative breaking occurs in the tree-level approximation in one specific four-family case. We take $m_{t}=165 \mathrm{GeV}, \tan \beta=1.5$, $m_{t^{\prime}}=100 \mathrm{GeV}, m_{b^{\prime}}=100 \mathrm{GeV}$ and $m_{\tau^{\prime}}=m_{\nu^{\prime}}=50 \mathrm{GeV}$. As outlined in the introduction, we specify our soft-SUSY-breaking parameter boundary conditions in terms of $\xi_{0} \equiv m_{0} / m_{1 / 2}, \xi_{A}=A / m_{1 / 2}$ and $m_{1 / 2}$. For this illustration we have adopted the string-motivated dilaton boundary condition scenario specified 
by $\xi_{0}=1 / \sqrt{3}, \xi_{A}=-1$. In the plots, $m_{H_{1}}^{2}, m_{H_{2}}^{2}$ are the Higgs mass-squared parameters appearing in the soft-SUSY-breaking potential (at $M_{U}$ they are equal to $m_{0}^{2}$ ), while $m_{1,2}^{2}=m_{H_{1,2}}^{2}+\mu^{2}$ are the parameters multiplying the $H_{1}^{2}$ and $H_{2}^{2}$ quadratic scalar field terms in the full scalar field potential; as defined earlier, $m_{3}^{2}$ is the coefficient of the $H_{1} H_{2}$ mixing term in the scalar field potential. In Fig. 5, we see that the $m_{H_{i}}^{2}$ start out above zero at $Q=M_{U}$, and then evolve below $M_{U}$ so that both eventually take on negative values (but with $m_{H_{2}}^{2}$ being the more negative). The $\mu$ parameter is determined at the weak scale by minimization of the RGE-improved tree-level Higgs potential, and is then evolved up to $M_{U}$. The fact that $\mu^{2}\left(m_{Z}\right)>0$ for the chosen value of $\tan \beta$ and the given value of $m_{Z}$ indicates that an allowed four-family EW symmetry breaking solution via the radiative breaking mechanism exists.

However, some differences in comparison to $N_{g}=3$ are apparent. Even for the small $\tan \beta=1.5$ value, $m_{H_{1}}^{2}$ has significant evolution due to the large $\lambda_{b^{\prime}}$ and $\lambda_{\tau^{\prime}}$ Yukawa couplings. In the standard three-family case, $m_{H_{1}}^{2}$ only evolves significantly when $\tan \beta$ is so large as to require a large value for $\lambda_{b}$. In Fig. 5b, one can see that although the scalar potential parameters $m_{1}^{2}, m_{2}^{2}$ and $m_{3}^{2}$ each evolves separately and ends with a value that is $>0$, in combination they serve to trigger the breaking of the EW symmetry, measured by the tree-level stability condition $\mathcal{S}=m_{1}^{2} m_{2}^{2}-m_{3}^{4}$. It is of course possible for the mechanism to fail, i.e. if $\mu^{2}<0$. This can happen in the case where $m_{H_{1}}^{2}$ is too large compared to $m_{H_{2}}^{2}$.

\section{The $\mathrm{N}_{\mathrm{g}}=4$ Higgs Sector}

We begin ${ }^{\star}$ by reminding the reader that at tree-level the Higgs sector is determined by just two parameters, $\tan \beta$ and $m_{A^{0}}$ (the mass of the CP-odd scalar Higgs boson), and that if $m_{A^{0}}$ is large (as we shall see it is in all the SUGRA models considered) then the lightest CP-even Higgs boson $h^{0}$ is very SM-like and has mass bounded from above by $m_{Z}$. However, it is well-known that one-loop radiative corrections to $m_{h^{0}}$ can significantly increase the upper limit of $m_{h^{0}}$ (denoted $m_{h^{0}}^{\max }$ ) for the large $m_{t}$ value found in the CDF and D0 experiments. Exactly how large the upper bound is depends upon other SUSY parameters, the most important sensitivity being to the stop squark mass, $m_{\tilde{t}}$. In the absence of a fourth generation, if the running masses are $m_{\widetilde{t}}=1 \mathrm{TeV}$ and $m_{t}=165 \mathrm{GeV}$, then $m_{h^{0}}$ can be as large as $120-125 \mathrm{GeV}$ at large $\tan \beta$. However, in many SUGRA models (for example the dilaton scenario) $m_{0}$ and, consequently, $m_{\widetilde{t}}$ are significantly smaller than $1 \mathrm{TeV}$ for $m_{\tilde{g}}$ values below $1 \mathrm{TeV}$. In such models $m_{h^{0}}^{\max }$ is typically $\lesssim 100 \mathrm{GeV}$.

* An excellent brief overview of Higgs phenomenology and discovery techniques is now available in the Higgs subgroup summary appearing in Ref. [23]. A longer version of this review will soon be available, Ref. [24]. References for statements not explicitly referenced below can be found in these reports. 
The magnitude of $m_{A^{0}}$ determines the observability of the $A^{0}$ and $H^{0}$ Higgs bosons, which are more or less degenerate when $m_{A^{0}}$ is large. For example, $e^{+} e^{-}$ colliders can only probe up to $m_{A^{0}} \sim m_{H^{0}} \sim \sqrt{s} / 2-30 \mathrm{GeV}$ in the $Z^{*} \rightarrow H^{0} A^{0}$ mode (the only viable mode when $h^{0}$ is SM-like). For $N_{g}=3$, SUGRA model predictions for $m_{A^{0}}$ can range from $200 \mathrm{GeV}$ on up, and an $e^{+} e^{-}$collider with $\sqrt{s}=500 \mathrm{GeV}$ would at least have a small chance of seeing $H^{0}+A^{0}$ production. The parameter $\mu$ is also of interest in that its origin in the SUGRA models is rather uncertain. For $N_{g}=3, \mu$ tends to take on rather moderate values $\sim 500 \mathrm{GeV}^{[16]}$ We now discuss the influence of a fourth generation.

First, we note that a fourth family will give an additional set of $\tau^{\prime}, \widetilde{\tau}^{\prime}, \nu^{\prime}, \widetilde{\nu}^{\prime}$, $b^{\prime}, \widetilde{b}^{\prime}$ and $t^{\prime}, \widetilde{t^{\prime}}$ loop contributions to the radiative corrections for $m_{h^{0}}^{\max }$. (As noted earlier, as a consequence of the many loop corrections for $N_{g}=4$ it is essential that the one-loop effective potential be used in the scalar potential minimization as well as in the determination of the Higgs masses.) A fourth family will also typically lead to a rather high $m_{\widetilde{g}}$ scale, as discussed in the following sections; this will in turn influence $m_{A^{0}}$ and $\mu$, which will often take on relatively large values.

Typical results for $\mu, m_{A^{0}}$ and $m_{h^{0}}$ are illustrated in Fig. 6, where we display contours of constant $\mu, m_{A^{0}}$ and $m_{h^{0}}$ in $m_{t^{\prime}}, m_{b^{\prime}}$ parameter space for $m_{t}\left(m_{t}\right)=$ $165 \mathrm{GeV}, \tan \beta=1.5$ and $m_{1 / 2}=600 \mathrm{GeV}$ in the dilaton scenario: $\xi_{0}=1 / \sqrt{3}$, $\xi_{A}=-1$. We see that $\mu$ is generally above $1 \mathrm{TeV}$, a substantial increase over the corresponding $N_{g}=3$ dilaton scenario result; $m_{A^{0}}$ is typically also rather large, ranging from $\sim 600 \mathrm{GeV}$ to above $1 \mathrm{TeV}-\mathrm{a} \sqrt{s}=500 \mathrm{GeV} e^{+} e^{-}$collider would not allow detection of the $Z^{*} \rightarrow H^{0} A^{0}$ pair production process. The large values of $m_{A^{0}} \sim m_{H^{0}}$ imply that one must also be cautious to account for supersymmetric decays of the $A^{0}$ and $H^{0}$. These decays can deplete the more easily observed $b \bar{b}$ decay channel, especially given that $\tan \beta$ cannot be large for $N_{g}=4$, implying that the $H^{0}, A^{0} \rightarrow b \bar{b}$ coupling cannot be greatly enhanced relative to the SM-like result. We will not attempt a detailed study of the $H^{0}$ and $A^{0}$ decays here.

The above results are not greatly altered by changing the $M_{U}$ boundary conditions, keeping $m_{1 / 2}$ fixed at $600 \mathrm{GeV}$. In the high- $m_{0}\left(\xi_{0}=1\right)$ scenario, both $m_{A^{0}}$ and $\mu$ become somewhat $(\sim 100 \mathrm{GeV})$ larger over most of the $m_{t^{\prime}}, m_{b^{\prime}}$ parameter space. The only exception is the corner where $m_{b^{\prime}}$ is big and $m_{t^{\prime}}$ is small; in this corner $m_{A^{0}}$ is slightly smaller $(564 \mathrm{GeV}$ compared to $610 \mathrm{GeV}$ ) in the high$m_{0}$ scenario than in the dilaton scenario. In the case of the no-scale boundary conditions, $\xi_{0}=\xi_{A}=0$, both $\mu$ and $m_{A^{0}}$ move to lower values (shifts are of order $50-100 \mathrm{GeV}$ ). For example, in the small $m_{t^{\prime}}$, large $m_{b^{\prime}}$ corner one finds $m_{A^{0}} \sim 450 \mathrm{GeV}$.

Fortunately as regards the prospects for $h^{0}$ detection, the extra loops from the fourth family do not yield overwhelmingly large radiative corrections to $m_{h^{0}}^{\max }$. They increase $m_{h^{0}}^{\max }$ by 10 to $25 \mathrm{GeV}$ relative to corresponding $N_{g}=3$ predictions. As illustrated by the contours in Fig. 6, at worst $m_{h^{0}} \max \sim 130 \mathrm{GeV}$ at the perturbative boundary in the $m_{b^{\prime}}, m_{t^{\prime}}$ parameter space. For all the three $M_{U}$ scenarios, $m_{h^{0}}$ remains very much in the $115-122 \mathrm{GeV}$ range for most of allowed 
$m_{b^{\prime}}, m_{t^{\prime}}$ parameter space, for this relatively large $m_{1 / 2}=600 \mathrm{GeV}$ value. (Lower $m_{h^{0}}$ values are predicted at lower $m_{1 / 2}$.) The phenomenology of the $h^{0}$ depends upon whether or not it is SM-like. For the bulk of parameter space, and certainly for the preferred scenarios and portions of parameter space, $m_{A^{0}}$ is large and the $h^{0}$ will be very SM-like. We discuss its phenomenology assuming that this is the case.

Because $m_{h^{0}}$ is predicted to be $\gtrsim 100 \mathrm{GeV}$ for many $N_{g}=4$ scenarios, LEP II would be less likely to find the $h^{0}$ if there is a fourth family. However, a $\sqrt{s}=$ $500 \mathrm{GeV} e^{+} e^{-}$collider would have no difficulty in doing so in the $Z^{*} \rightarrow Z h^{0}$ production mode. At the $\mathrm{TeV}^{\star}$ upgrade of the Tevatron, detection of a SM-like $h^{0}$ is probably only possible in the $W h^{0} \rightarrow \ell \nu b \bar{b}$ mode, and then only if $m_{h^{0}} \lesssim$ $95 \mathrm{GeV}$. (Although it is not impossible that the $W h^{0} \rightarrow \ell \nu \tau^{+} \tau^{-}$mode could be used for $110 \lesssim m_{h^{0}} \lesssim 120 \mathrm{GeV}^{[25]}$ ) For the many four-family scenarios that lead to $m_{h^{0}}$ above $100 \mathrm{GeV}$, searches for the $h^{0}$ at the Tevatron would be, at best, problematical.

At the LHC, a SM-like Higgs boson (for which we use the generic notation $h$ below - the $h^{0}$ might or might not be perfectly SM-like) in the mass region being discussed would typically be found through production via $g g \rightarrow h$ and decay to either $\gamma \gamma$ or $Z Z^{*}$ (with $Z Z^{*} \rightarrow 4 \ell$ ). The $g g \rightarrow h$ production rate, proportional to $\Gamma(h \rightarrow g g)$ would be greatly enhanced by the additional $t^{\prime}$ and $b^{\prime}$ loop contributions to the one-loop $g g \rightarrow h$ coupling. This is illustrated in Fig. 7 where we plot for a SM-like $h$ the ratio of $N_{g}=4$ to $N_{g}=3$ values for: $\Gamma(h \rightarrow g g), \Gamma(h \rightarrow \gamma \gamma)$, and $\Gamma(h \rightarrow g g) \times B R(h \rightarrow \gamma \gamma)$, taking $\tan \beta=1.5$, $m_{t}=165 \mathrm{GeV}, m_{t^{\prime}}=m_{b^{\prime}}=100 \mathrm{GeV}, m_{\tau^{\prime}}=m_{\nu^{\prime}}=50 \mathrm{GeV}$, and assuming that superpartners are sufficiently heavy that their contributions to these one-loop quantities are small. (As discussed in Sec. 7a, experimental limits tend to prefer this type of scenario.) For $\Gamma(h \rightarrow g g)$, and hence the $g g \rightarrow h$ production rate, we see an enhancement by a factor of 10 . Thus, the $4 \ell$ channel, which for $N_{g}=3$ is only viable for $m_{h} \gtrsim 130 \mathrm{GeV}$, would yield a detectable signal down to somewhat lower masses, perhaps as low as $m_{h}=120 \mathrm{GeV}$. $\left(B R\left(h \rightarrow Z Z^{*}\right)\right.$ falls very rapidly with decreasing $m_{h}$ so very few events would result for $m_{h}$ values much below this.)

The $h \rightarrow \gamma \gamma$ decay also arises at one-loop. For $N_{g}=3$, the main contribution is from the $W$-loop diagram. Fermion loops (for massive fermions) cancel against the $W$-loop contribution and decrease the $h \rightarrow \gamma \gamma$ width. For $N_{g}=4$ this cancellation can be quite substantial, as illustrated in Fig. . $^{[26]}$ Combining the resulting reduction in $B R(h \rightarrow \gamma \gamma)$ with the enhanced $g g \rightarrow h$ production rate, the resulting $\gamma \gamma$ channel event rate tends to be substantially suppressed relative to the $N_{g}=3$ rate in the $100-130 \mathrm{GeV}$ mass range of interest. In fact, the preferred $m_{t^{\prime}}, m_{b^{\prime}}, m_{\tau^{\prime}}$ mass choices delineated above are about the worst that can be made in this regard. Thus, detection of the $h^{0}$ in the $\gamma \gamma$ channel at the LHC becomes problematical, even when $m_{A^{0}}$ is large and the $h^{0}$ is SM-like.

Not investigated to date is whether the enhanced production rate from $g g \rightarrow h$ might make detection of a SM-like $h^{0}$ in the inclusive $b \bar{b}$ channel possible (assuming 
high $b$-tagging efficiency and purity). Finally, we note that the extra family has essentially no impact on the $p p \rightarrow t \bar{t} h^{0} \rightarrow \ell b \bar{b} b \bar{b} X$ LHC detection mode, which would continue to be viable for $m_{h^{0}} \lesssim 120 \mathrm{GeV}$, when the $h^{0}$ is SM-like. ${ }^{[23]}$

Regarding the heavier $A^{0}$ and $H^{0}$, for our typical scenario they are so massive that perhaps the only accelerator with adequate energy for their production and possible detection will be the LHC. At the LHC, for $N_{g}=3$ the detection of a massive $A^{0}$ or $H^{0}$ is possible only if $\tan \beta$ is so large $(e . g . \gtrsim 10)$, that the $g g \rightarrow b \bar{b} H^{0}$ and $g g \rightarrow b \bar{b} A^{0}$ production rates significantly exceed the inclusive $g g \rightarrow H^{0}, A^{0}$ rates $^{[23,24]}$ However, for $N_{g}=4$ the $b^{\prime}$ and $t^{\prime}$ loop contributions to $g g \rightarrow A^{0}$ and $g g \rightarrow H^{0}$ will greatly increase these inclusive production rates, regardless of the $\tan \beta$ value. The possibility of observing the $H^{0}$ and $A^{0}$ at the LHC in the inclusive $b \bar{b}$ final state assuming such highly enhanced rates should be carefully examined.

As a final aside, we note that the standard relation, ${ }^{[27]}$

$$
m_{A^{0}}^{2} \simeq \frac{m_{\widetilde{\nu}}^{2}+\mu^{2}}{\sin ^{2} \beta}
$$

valid for $\tan \beta \lesssim 20$ if $N_{g}=3$, is not necessarily maintained for $N_{g}=4$ since there will be corrections involving the $\lambda_{b^{\prime}}, \lambda_{\tau^{\prime}}, \lambda_{b}$ Yukawas (where $\lambda_{b}$ can essentially be neglected). In Fig. 6, for $m_{t^{\prime}}, m_{b^{\prime}}$ large, e.g. $m_{t^{\prime}}=m_{b^{\prime}}=100 \mathrm{GeV}$, the relation is satisfied to within $3 \%$; however for $m_{b^{\prime}} \simeq 110 \mathrm{GeV}, m_{t^{\prime}}=55 \mathrm{GeV}$, the relation is seriously violated: $\left(m_{A^{0}}^{2} \sin ^{2} \beta\right) /\left(m_{\widetilde{\nu}}^{2}+\mu^{2}\right) \simeq 0.25$. This latter situation is realized in the region where $m_{b^{\prime}}>m_{t^{\prime}}$, a region that is experimentally disfavored unless an unnatural quark mixing pattern exists (see Ref. [1] and Sec. 7 for a more detailed discussion of this point).

\section{A Fourth-Generation Sparticle Constraint on the SUSY Scale}

We turn now to an important additional constraint on soft-SUSY-breaking parameters that can arise from consistency of the sparticle mass spectrum with LEP limits and a neutral LSP (lightest supersymmetric particle). (A charged LSP is excluded experimentally.) A glance at Eqs. (A.11) through (A.19) in the Appendix shows that $d m_{i}^{2} / d t$ (where $t=\frac{1}{2 \pi} \ln [Q(\mathrm{GeV})]$ ) for squarks and sleptons receives positive contributions from Yukawa terms and negative contributions from gauge terms. Thus, starting from a universal $m_{0}^{2}$ and evolving downwards in $t$ to $m_{Z}$, the lightest squark/slepton will be the one with the largest Yukawa contributions relative to gauge contributions. This turns out to always be the $\widetilde{\tau}_{R}^{\prime}$ or the $\widetilde{\nu}_{R}^{\prime}$. After including $\widetilde{\tau}_{R}^{\prime}-\widetilde{\tau}_{L}^{\prime}$ mixing, the lightest $\widetilde{\tau}^{\prime}$ eigenstate is denoted $\widetilde{\tau}_{1}^{\prime}$, and similarly $\widetilde{\nu}_{1}^{\prime}$ is the lightest $\widetilde{\nu}^{\prime}$ eigenstate.

The $\widetilde{\nu}_{1}^{\prime}$ can be even lighter than the $\widetilde{\tau}_{1}^{\prime}$ in scenarios with small $\xi_{0}$, such as the dilaton and no-scale models. However, we see no general phenomenological 
reason for not allowing the $\widetilde{\nu}_{1}^{\prime}$ to be the LSP. Indeed, it is even quite likely that the $\widetilde{\chi}_{1}^{0}$ decays invisibly when $m_{\widetilde{\chi}_{1}^{0}}>m_{\widetilde{\nu}_{1}^{\prime}}$ via $\widetilde{\chi}_{1}^{0} \rightarrow \nu_{\tau} \widetilde{\nu}_{1}^{\prime}$, assuming at least a small non-zero value for the required $3-4$ generation mixing angle. Thus, we will only impose a phenomenological limit on the charged $\widetilde{\tau}_{1}^{\prime}$.

Eq. (A.15) shows that the Yukawa contributions to $d m_{\widetilde{\tau}_{R}^{\prime}}^{2} / d t$ (in the Appendix we use the notation $\widetilde{\tau}_{R}^{\prime}=E^{\prime}$ ) are controlled by $\mathcal{D}_{\tau^{\prime}}=m_{H_{1}}^{2}+m_{\widetilde{\tau}_{L}^{\prime}}^{2}+m_{\widetilde{\tau}_{R}^{\prime}}^{2}+A_{\widetilde{\tau}^{\prime}}^{2}$. For moderate initial values, $m_{\widetilde{\tau}_{L}^{\prime}}=m_{\widetilde{\tau}_{R}^{\prime}}=m_{0}$, the more negative $m_{H_{1}}^{2}$ is (see Fig. 5) the larger will be $m_{\widetilde{\tau}_{R}^{\prime}}^{2} ; m_{H_{1}}^{2}$ in turn becomes more negative for larger $m_{b^{\prime}}$. Thus, for some choices of soft-SUSY-breaking parameters it is possible that $m_{\widetilde{\tau}_{1}^{\prime}}$ will fall below the $45 \mathrm{GeV}$ LEP limit and/or below the mass of the lightest supersymmetric particle (the lightest neutralino, $\widetilde{\chi}_{1}^{0}$, in the models we consider) for low values of $m_{b^{\prime}}$ (but not for higher values).

To illustrate this, we return to Fig. 6 , where we have chosen $\tan \beta=1.5$, $m_{t}\left(m_{t}\right)=165 \mathrm{GeV}$ and dilaton boundary conditions for the soft-SUSY-breaking parameters with $m_{1 / 2}=600 \mathrm{GeV}$. The diamonds indicate the portion of parameter space at low $m_{b^{\prime}}$ that is ruled out because one predicts $m_{\widetilde{\tau}_{1}^{\prime}}<45 \mathrm{GeV}$ or $m_{\widetilde{\tau}_{1}^{\prime}}<m_{\widetilde{\chi}_{1}^{0}}$. (For this particular choice of $m_{1 / 2}, m_{\widetilde{\chi}_{1}^{0}} \sim 108 \mathrm{GeV}$ and it is the $m_{\widetilde{\tau}_{1}^{\prime}}>m_{\widetilde{\chi}_{1}^{0}}$ requirement that fixes the diamond region.) By raising $m_{1 / 2}$, this problem region is moved to lower $m_{b^{\prime}}$ values since the starting value of $m_{0}^{2} \propto m_{1 / 2}^{2}$ for $m_{\widetilde{\tau}_{R}^{\prime}}^{2}$ is increased more rapidly than the off-diagonal mixing term $A \propto m_{1 / 2}$. Conversely, by lowering $m_{1 / 2}$ we eventually reach a value for which no portion of parameter space remains allowed.

The type of boundary condition applied is also important. For instance, for the same $m_{1 / 2}=600 \mathrm{GeV}$ but $\xi_{0}=1, \xi_{A}=-1$ (the high- $m_{0}$ scenario) no points with $m_{b^{\prime}}, m_{t^{\prime}}>50 \mathrm{GeV}$ are eliminated by virtue of the $\widetilde{\tau}_{1}^{\prime}$ constraint, whereas for the no-scale choice of $\xi_{0}=\xi_{A}=0$, the portion of parameter space removed expands to include slightly higher values of $m_{b^{\prime}}$ than in the dilaton scenario.

Let us further expand upon this point. From the above discussion we see that in the generic one-loop formula, $m_{i}^{2}=m_{0}^{2}+\hat{C}_{i} m_{1 / 2}^{2}+D_{i} m_{Z}^{2} \cos 2 \beta$, the evolution parameter $\hat{C}_{i}$ for $i=\widetilde{\tau}_{R}^{\prime}$ is strongly affected by indirect effects from $\lambda_{b^{\prime}}$, due to the absence of an $\alpha_{s}$ contribution to $\hat{C}_{i}$. In general, larger $m_{b^{\prime}}$ tends to raise $\hat{C}_{\widetilde{\tau}_{R}^{\prime}}$. As we have discussed above, this can be traced to an increasingly negative contribution in the RGE for $m_{\widetilde{\tau}_{R}^{\prime}}^{2}$ from the increasingly negative value of $m_{H_{1}}^{2}$ as $m_{b^{\prime}}$ increases.

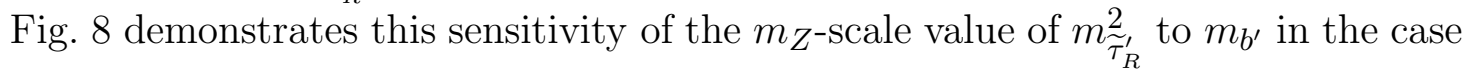
of $m_{t^{\prime}}=100 \mathrm{GeV}, \tan \beta=1.5, m_{1 / 2}=600 \mathrm{GeV}$ with dilaton boundary conditions: as $m_{b^{\prime}}$ is lowered from $m_{b^{\prime}}=110 \mathrm{GeV}$ to $m_{b^{\prime}}=90 \mathrm{GeV}$, the $m_{Z^{\prime}}$-scale value of $m_{\widetilde{\tau}_{R}^{\prime}}^{2}$ is lowered by $\sim 40 \%$, with the lowest $\widetilde{\tau}^{\prime}$ physical eigenstate mass decreasing dramatically, from $m_{\widetilde{\tau}_{1}^{\prime}}=311 \mathrm{GeV}$ to $189 \mathrm{GeV}$. In contrast, the evolution and 


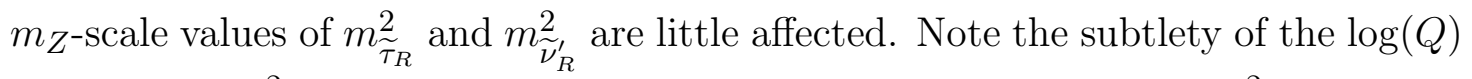
behavior of $m_{\widetilde{\tau}_{R}^{\prime}}^{2}$. In the region of small (and decreasing) $\log (Q) m_{\widetilde{\tau}_{R}^{\prime}}^{2}$ rises due to the increasingly more negative value for $m_{H_{1}}^{2}$ as $\log (Q)$ decreases (see Fig. 5). Since this rise is less for smaller $m_{b^{\prime}}$, for small enough $m_{b^{\prime}}$ one can even obtain $m_{\widetilde{\tau}_{R}^{\prime}}^{2}<0$, and EM will no longer be unbroken. However, as $m_{b^{\prime}}$ is decreased one first arrives at a point where either $m_{\widetilde{\tau}_{1}^{\prime}}<m_{\widetilde{\chi}_{1}^{0}}$ and the LSP is no longer neutral, or $m_{\widetilde{\tau}_{1}^{\prime}}<45 \mathrm{GeV}$, violating LEP limits. We re-emphasize that these requirements result in the strongest additional $R G E$-related phenomenological constraint deriving from the SUGRA extension of the four-family model.

To gain some additional insight regarding the stringency of this bound as a function of $M_{U}$-scale boundary conditions, we present the right-hand boundaries, analogous to that of the diamond region shown in Fig. 6, for a selection of different possibilities in Fig. 9. The right-hand window shows results for the dilaton case of $\left(\xi_{0}, \xi_{A}\right)=(1 / \sqrt{3},-1)$ for $m_{1 / 2}=900,600,300,250 \mathrm{GeV}$. Very little of the Yukawaallowed parameter space survives for the lowest choice. In the left-hand window we fix $m_{1 / 2}=400 \mathrm{GeV}$ and vary $\left(\xi_{0}, \xi_{A}\right)$. For the no-scale choice of $(0,0)$ very little of parameter space yields an acceptable $\widetilde{\tau}_{1}^{\prime}$.

Of course, the $m_{\widetilde{\tau}_{1}^{\prime}}>\max \left\{45 \mathrm{GeV}, m_{\widetilde{\chi}_{1}^{0}}\right\}$ constraint is significant even if we adopt a large (but allowed) value of $m_{b^{\prime}}$ and do not employ a specific scenario for $\xi_{0}$ and $\xi_{A}$. This is illustrated in Fig. 10. There, we take $(m(m)$ masses) $m_{t}=165 \mathrm{GeV}, m_{t^{\prime}}=m_{b^{\prime}}=100 \mathrm{GeV}, m_{\tau^{\prime}}=m_{\nu^{\prime}}=50 \mathrm{GeV}$ and $\tan \beta=1.5$ (for these mass choices, solutions are only allowed for $\tan \beta$ values very near 1.5) and plot the smallest possible value for $\xi_{0}=m_{0} / m_{1 / 2}$ that is allowed as a function of $m_{1 / 2}$ after scanning over $\xi_{A}$ in the range $[-3,+3]$. A very important generic feature emerges from the steep rise of the minimum $\xi_{0}$ value as $m_{1 / 2}$ decreases: for given $m_{t^{\prime}}, m_{b^{\prime}}, m_{\tau^{\prime}}, m_{\nu^{\prime}}$ there is a definite lower bound on $m_{1 / 2}$ (for reasonable values of $\left.\xi_{0}\right)$ arising from the $m_{\widetilde{\tau}_{1}^{\prime}}>\max \left\{45 \mathrm{GeV}, m_{\widetilde{\chi}_{1}^{0}}\right\}$ constraint. For the $t^{\prime}$ and $b^{\prime}$ masses considered, this bound is $m_{1 / 2} \gtrsim 140 \mathrm{GeV}$. If we recall that $m_{\tilde{g}} \sim\left(\alpha_{3}\left(m_{Z}\right) / \alpha_{U}\right) m_{1 / 2}$, and that $\alpha_{3}\left(m_{Z}\right) / \alpha_{U} \sim 1.4$, we see that this $m_{\tilde{g}}$ bound translates into a significant lower bound of $m_{\tilde{g}} \gtrsim 200 \mathrm{GeV}$ deriving purely from limits on the fourth-generation $\widetilde{\tau}_{1}^{\prime}$ mass. This type of constraint does not arise in the $N_{g}=3$ MSSM.

The ultimate lower bound on $m_{1 / 2}$ (along with the corresponding lower bound on $m_{\tilde{g}}$ ) is actually quite independent of $m_{b^{\prime}}$. For instance, if $m_{b^{\prime}}$ is lowered to $50 \mathrm{GeV}$, keeping $m_{t}$ and $m_{t^{\prime}}$ fixed, at $\xi_{0}=5$ the lowest allowed value of $m_{1 / 2}$ decreases by only about $5 \mathrm{GeV}$ compared to the $m_{b^{\prime}}=100 \mathrm{GeV}$ value of $\sim 140 \mathrm{GeV}$ illustrated in Fig. 10. This is because, at the ultimate lower bound, $m_{0}$ is very large and the $m_{H_{1}}^{2}$ term in $\mathcal{D}_{\tau^{\prime}}$ (see above) is swamped by the $m_{\widetilde{\tau}_{R}^{\prime}}^{2}$ and $m_{\widetilde{\tau}_{L}^{\prime}}^{2}$ terms which start off of order $m_{0}^{2}$ and remain large. 
We re-emphasize the fact that in a specific SUGRA scenario with a fixed value of $\xi_{0}$ (and of $\xi_{A}$ ) the lower bound on $m_{1 / 2}$ (and $m_{\tilde{g}}$ ) can be much larger than the high $-\xi_{0}$ ultimate lower bound if $\xi_{0}$ is small, as is illustrated in Fig. 10. (Of course, whatever the lower bound on $m_{\tilde{g}}\left(m_{\tilde{g}}\right), m_{\tilde{g}}$ (pole) will be roughly 5 -6\% higher.) We also note that at fixed $\xi_{0}$ there is some dependence of the lower bound on $m_{1 / 2}$ upon the value of $\xi_{A}$. This dependence is extremely weak at high $\xi_{0}$, where $\xi_{A}$ values in the entire $[-3,+3]$ range generally give an allowed solution for the lowest acceptable $m_{1 / 2}$ value. But for lower $\xi_{0}$ values, the lower bound on $m_{1 / 2}$ is generally achieved only for $\xi_{A}$ values near 0 . (In fact, the appropriate $\xi_{A}$ range more or less scales with the magnitude of $\xi_{0}$.)

Perhaps one other plot is useful in fully understanding the $\widetilde{\tau}_{1}^{\prime}$ constraint. In Fig. 11 we consider various quantities as a function of $\xi_{A}$ (ranging from -3 to +3 ) for fixed values of $\xi_{0}$ in the range from 0 to 5 . In all cases we take $m_{b^{\prime}}\left(m_{b^{\prime}}\right)=$ $m_{t^{\prime}}\left(m_{t^{\prime}}\right)=100 \mathrm{GeV}$ (corresponding to pole masses of approximately $105 \mathrm{GeV}$ ). For each $\xi_{0}, \xi_{A}$ choice, we determine the minimum value of $m_{1 / 2}$ (the scan being confined to the region $m_{1 / 2} \leq 10 \mathrm{TeV}$ ) that is consistent with the $\tilde{\tau}_{1}^{\prime}$ constraint. We plot the (minimum) value of $m_{\widetilde{g}}\left(m_{\tilde{g}}\right)$ corresponding to this minimum value of $m_{1 / 2}$. (For later phenomenological use, we also plot the corresponding $m_{h^{0}}$ and $m_{b^{\prime}}($ pole $)-m_{h^{0}}-m_{b}$ values.) For curves of limited extent in $\xi_{A}$, the termination point(s) define the range beyond which consistent solutions are not found with $m_{1 / 2} \leq 10 \mathrm{TeV}$. The inconsistencies that arise at large $\xi_{A}$ are of two types: (i) that the EWSB solution requires $m_{A^{0}}^{2}<0$; and/or (ii) that a 4th-family sparticle with color or charge must have $m^{2}<0$, thereby breaking the color and/or U(1)electromagnetic symmetries.

This plot defines precisely the minimum $m_{\tilde{g}}$ value that one can have for a given $\xi_{0}, \xi_{A}$ boundary condition choice. Once again we see an absolute lower limit of order $m_{\widetilde{g}}\left(m_{\tilde{g}}\right) \gtrsim 200 \mathrm{GeV}$ for our choice of $m_{b^{\prime}}, m_{t^{\prime}}$; the value of $\tan \beta$ for which the minimum $m_{\tilde{g}}$ value is reached is always in the range 1.5 to 1.6. Note that for low $\xi_{0}$, the allowed solution range for $\xi_{A}$ is limited, and that the minimum $m_{\tilde{g}}$ achievable increases substantially when $\xi_{A}$ is not near 0 .

\section{The Sparticle Spectrum}

We shall find in Sec. 7 that the first evidence for a fourth family is very likely to be discovery of the $b^{\prime}, t^{\prime}, \tau^{\prime}$ and/or $\nu^{\prime}$ at LEP-II and/or the Tevatron. If one or more of these fourth-family members are found, the immediate question will be how this impacts the supersymmetric particle spectrum, especially in the standard renormalization group equation (RGE) context. It is this latter issue that we address in this section. First, we highlight the main features of the sparticle spectrum in a four-family scenario, and compare the results to those obtained in the three-family case. As previewed in Sec. 4, we find that it is more than likely that some of the fourth generation squarks and/or sleptons will be lighter than their counterparts in the first three generations, with the $\widetilde{\tau}_{1}^{\prime}$ most probably being 
the lightest. However, testing consistency of their masses with unification and the RGE's is likely to be challenging given the possibly large $A$-term induced mixing, and the probability that they will have strange decay patterns and be tricky to observe. Thus, we will focus primarily on gaugino masses and on the masses of the sleptons and squarks of the first two generations, focusing on when and how correlations among these masses will be indirectly sensitive to the presence of a fourth generation through the RGE's. Certain relationships between masses are rather insensitive to whether $N_{g}=4$ or $N_{g}=3$, and thus provide a test of the general RGE context and universality of boundary conditions, while other mass correlations are very different depending upon the value of $N_{g}$. These latter relationships with strong $N_{g}$ dependence would provide indirect evidence for the presence of a fourth generation, even if no particle or sparticle belonging to the fourth generation is directly observed.

We first present some sample mass spectra, then discuss mass sum rules and relations, and finally focus on a specific correlation between the first-family slepton masses and the LSP mass that could reveal the presence of a fourth generation.

\section{6a. Sample Mass Spectra}

For our illustrations we shall adopt $\mu>0, m_{t^{\prime}}=m_{b^{\prime}}=100 \mathrm{GeV}$, and $m_{\tau^{\prime}}=$ $m_{\nu^{\prime}}=50 \mathrm{GeV}$. We scan over the allowed $\tan \beta$ values at any given choice for $m_{\widetilde{g}}\left(m_{\tilde{g}}\right)$ and plot mass spectra in units of $m_{\tilde{g}}\left(m_{\tilde{g}}\right)$. Three $M_{U}$ boundary conditions will be considered:

1. the dilaton scenario, with $\xi_{A} \equiv A / m_{1 / 2}=-1, \xi_{0} \equiv m_{0} / m_{1 / 2}=1 / \sqrt{3}$;

2. the no-scale scenario, with $\xi_{A}=\xi_{0}=0$; and

3. the high- $m_{0}$ scenario, with $\xi_{A}=-1, \xi_{0}=1$.

We begin by focusing on the spectrum for the $\widetilde{\tau}_{1}^{\prime}$ for these three models.

In Fig. 12, we plot the $m_{\widetilde{\tau}_{1}^{\prime}}$ spectra obtained by scanning over allowed $\tan \beta$ values at various $m_{\tilde{g}}$ values. The lower limit on $m_{\widetilde{\tau}_{1}^{\prime}}$ is fixed by $m_{\widetilde{\tau}_{1}^{\prime}}>45 \mathrm{GeV}$ in the case of the high- $m_{0}$ scenario, and by $m_{\widetilde{\tau}_{1}^{\prime}}>m_{\widetilde{\chi}_{1}^{0}}$ in the no-scale and dilaton models. The dramatic decrease of $m_{\widetilde{\tau}_{1}^{\prime}}$ as $m_{\widetilde{g}}$ decreases, discussed in Sec. 5, is evident. Of course, at high $m_{\tilde{g}}$ values, the value of $m_{\widetilde{\tau}_{1}^{\prime}}$ is given roughly by $m_{\widetilde{\tau}_{1}^{\prime}}^{2} \sim$ $m_{0}^{2}+\hat{C}_{\widetilde{\tau}_{1}^{\prime}} m_{1 / 2}^{2} \propto\left(\xi_{0}^{2}+\hat{C}_{\widetilde{\tau}_{1}^{\prime}}\right) m_{\widetilde{g}}^{2}$, which is larger for larger values of $\xi_{0}$.

Let us now turn to the typical mass spectra for all the other supersymmetric particles. We begin with results for dilaton boundary conditions: Figure 13 shows our results. These can be compared to the $N_{g}=3$ results from Ref. [5] for this 
same boundary condition choice.

The first big difference between the $N_{g}=4$ and $N_{g}=3$ cases is the much larger lower bound on $m_{\widetilde{g}}$ in the former case. As discussed previously, this reflects the $m_{\widetilde{\tau}_{1}^{\prime}}>\max \left\{45 \mathrm{GeV}, m_{\widetilde{\chi}_{1}^{0}}\right\}$ requirement that is violated for low $m_{\widetilde{g}}$ values. A less dramatic difference is that the allowed range of $\tan \beta$ is smaller for $N_{g}=4$, and thus the value of $m_{\tilde{g}}$ almost completely fixes the chargino and neutralino masses, whereas for $N_{g}=3$ there is some scatter. The limitation of $\tan \beta$ to low values for $N_{g}=4$ also means that (unlike for $N_{g}=3$ ) the $\widetilde{\tau}_{1}$ remains almost degenerate with the $\widetilde{\ell}_{R}$ 's of the first two families; thus, a separate plot for the $\widetilde{\tau}_{1}$ is not given in our figures. At a given $m_{\tilde{g}}$, the most significant $N_{g}=4$ vs. $N_{g}=3$ difference is the much larger masses for the squarks and sleptons of the first three generations. Finally, there is the simple fact that fourth-generation squarks and sleptons are present for $N_{g}=4$. We have already noted that $\widetilde{\tau}_{1}^{\prime}$ and $\widetilde{\nu}_{1}^{\prime}$ tend to be the lightest of the sleptons. From Fig. 13 we see that the lightest squark is very likely to be the $\widetilde{b}_{1}^{\prime}$. This is a rather general result.

As already hinted and more directly demonstrated in the next subsection, the large squark and slepton masses for the first three generation members can be directly traced to the much larger value of $\alpha\left(M_{U}\right)$ for $N_{g}=4$. The basic idea is that $m_{i}^{2}=m_{0}^{2}+\hat{C}_{i} m_{1 / 2}^{2}+D_{i} m_{Z}^{2} \cos 2 \beta$, where $i$ indicates the squark or slepton in question and $m_{1 / 2}=m_{\tilde{g}} \alpha\left(M_{U}\right) / \alpha_{3}\left(m_{\tilde{g}}\right)$. Since the $\hat{C}_{i}$ are not terribly different in the three- and four-generation cases, the much larger $\alpha\left(M_{U}\right)$ in the $N_{g}=4$ case greatly increases the squark or slepton mass at given $m_{\tilde{g}}$. Combining this effect with the higher lower bound for $m_{\tilde{g}}$ in the $N_{g}=4 \mathrm{vs}$. $N_{g}=3$ case results in a large increase in the lower bounds on squark and slepton masses in going to $N_{g}=4$. For example, $m_{\widetilde{\ell}_{L}}, m_{\widetilde{\nu}}, m_{\widetilde{\ell}_{R}} \gtrsim 250,240,200 \mathrm{GeV}$ compared to $m_{\tilde{\ell}_{L}}, m_{\widetilde{\nu}}, m_{\widetilde{\ell}_{R}} \gtrsim 110,52,45 \mathrm{GeV}$ in the $N_{g}=4$ and $N_{g}=3$ cases, respectively. The high lower bound values in the $N_{g}=4$ case place these sleptons from the first two families beyond the reach of LEP-II and almost beyond the reach of a $\sqrt{s}=$ $500 \mathrm{GeV}$ NLC. We shall see that the large squark and slepton masses relative to gaugino masses might well provide the most compelling indirect indication for the presence of four generations that one can obtain using only particles and sparticles belonging to the first three generations.

In Fig. 14 we give the corresponding results assuming no-scale boundary conditions: $\xi_{A}=\xi_{0}=0$. The first noteworthy point is the much higher lower bound on $m_{\widetilde{g}}$ that arises in the no-scale case, as compared to dilaton boundary conditions, when $N_{g}=4$. Due to the zero value of $m_{0}$ at $M_{U}$, the slepton masses

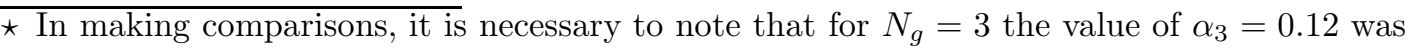
employed in Ref. [5], as compared to $\alpha_{3} \sim 0.128$ or so for our full two-loop four-generation treatment here. Since $M_{1}: M_{2}: M_{3} \sim \alpha_{1}: \alpha_{2}: \alpha_{3}\left(\right.$ all at $\left.m_{Z}\right), m_{\widetilde{\chi}_{1}^{0}}, m_{\widetilde{\chi}_{2}^{0}}, m_{\widetilde{\chi}_{1}^{+}}$masses at a given $m_{g}$ in Ref. [5] for $N_{g}=3$ are approximately $0.94 \sim 0.12 / 0.128$ times those appearing in Fig. 13. 
are smaller than for dilaton boundary conditions, and, in particular, $m_{\widetilde{\tau}_{1}^{\prime}}$ is more easily driven to too low a value. In addition, for the lower $m_{\tilde{g}}$ values allowed by the $m_{\widetilde{\tau}_{1}^{\prime}}>m_{\widetilde{\chi}_{1}^{0}}$ constraint, the $\widetilde{\nu}_{1}^{\prime}$ can be the LSP; it is often very substantially lighter than the $\widetilde{\chi}_{1}^{0}$. However, the $\widetilde{\ell}_{L}$ and $\widetilde{\nu}$ masses for the first two families are still much bigger than found in the comparison results for $N_{g}=3$, see Ref. [5]. The $\tilde{\ell}_{R}$ is significantly lighter than the $\tilde{\ell}_{L}$ in the no-scale scenario (whatever $N_{g}$ ) because the associated soft-mass-squared evolution is fed only by the $U(1)$ gaugino mass terms, and moves to positive values (starting at $M_{U}$ from 0 for $\xi_{0}=0$ ) much more slowly than the soft-mass-squared that contributes to the $\tilde{\ell}_{L}$ and $\widetilde{\nu}$ masses, which is fed by $S U(2)$ as well as $U(1)$ gaugino mass terms (see Sec. 6b). Squark masses remain very similar to the dilaton scenario results due to the fact that the $\hat{C}_{\widetilde{q}}$ terms dominate the $m_{0}$ terms for strongly-interacting sparticles. As in the dilaton case, the large squark and slepton masses relative to $m_{\tilde{g}}$ provide a signal for $N_{g}=4$. Again there is a large difference between $N_{g}=4$ and $N_{g}=3$ in the lower bounds so crucial for LEP and NLC phenomenology. For example, $m_{\tilde{\ell}_{L}}, m_{\widetilde{\nu}}, m_{\tilde{\ell}_{R}} \gtrsim 265,260,150 \mathrm{GeV}$ compared to $m_{\tilde{\ell}_{L}}, m_{\widetilde{\nu}}, m_{\tilde{\ell}_{R}} \gtrsim 110,60,70 \mathrm{GeV}$ for $N_{g}=4$ and $N_{g}=3$, respectively. Thus, for $N_{g}=4$ only the $\tilde{\ell}_{R}$ would be within the kinematical reach of a $\sqrt{s}=500 \mathrm{GeV}$ NLC.

In Fig. 15 we give results assuming the high- $m_{0}$ boundary condition case: $\xi_{0}=-\xi_{A}=1$. Here, the $\widetilde{\tau}_{1}^{\prime}$ constraint is more easily satisfied and $m_{\tilde{g}}$ can take on lower values. Even at the lowest allowed $m_{\widetilde{g}}$ values, the $\widetilde{\nu}_{1}^{\prime}$ is heavier than the $\widetilde{\tau}_{1}^{\prime}$ (see Fig. 12), which in turn is required to be heavier than the LSP; in fact, even at low $m_{\tilde{g}}$ the $\widetilde{\nu}_{1}^{\prime}$ (but not the $\widetilde{\tau}_{1}^{\prime}$ ) is heavier than the $\tilde{\chi}_{1}^{+}$. Squarks and sleptons receive a significant fraction of their mass from the large $m_{0}$ value, and are thus more similar in mass. However, the $\hat{C}_{\widetilde{q}}$ contributions are still very important, and squark and slepton masses for members of the first two families continue to be much larger for $N_{g}=4$ than for $N_{g}=3$. To repeat our previous sample comparisons, $m_{\widetilde{\ell}_{L}}, m_{\widetilde{\nu}}, m_{\widetilde{\ell}_{R}} \gtrsim 245,240,220 \mathrm{GeV}$ for $N_{g}=4$ compared to $m_{\tilde{\ell}_{L}}, m_{\widetilde{\nu}}, m_{\tilde{\ell}_{R}} \gtrsim 120,89,107 \mathrm{GeV}$ for $N_{g}=3$.

\section{6b. Sum Rule and Sparticle Spectrum Tests for $N_{g}=3$ vs. $N_{g}=4$}

In the models considered here, having universal soft-SUSY-breaking scalar mass $m_{0}$, there are many sum rules relating the gaugino, squark and slepton masses. Here, we survey the impact of a fourth family on the masses and mass sum rules for the gauginos and for the squarks and sleptons belonging to the first two families; ${ }^{[28]}$ we also point out several differences between the sum rules that relate only fourth-family masses and those that involve only the third-family masses. Generally speaking, because of the large mixings that can be present in both third and fourth generation squark and slepton mass matrices, the most precise tests of the consistency of RGE evolution and grand unification with sparticle mass spectra may be those employing first and second generation members, for which the mass matrices are very nearly diagonal. 
To proceed, we must first discuss gaugino masses in more detail. We employ the notation $k=1,2,3$ for the $U(1), S U(2)$, and $S U(3)$ groups; $g_{k}(t)$ are the associated running coupling constants, and $M_{k}(t)$ are the associated running gaugino masses, where $t=\frac{1}{2 \pi} \log [Q(\mathrm{GeV})]-t_{U}$ denotes $t$ at $Q=M_{U}, t_{Z}$ denotes $t$ at $Q=m_{Z}$, and so forth. If the gaugino masses do indeed take a universal value $m_{1 / 2}$ at $M_{U}$ then at one-loop we have the standard relations:

$$
M_{k}(t)=\left[\alpha_{k}(t) / \alpha\left(t_{U}\right)\right] m_{1 / 2}
$$

see Eqs. (A.28) through (A.31) in Appendix A. For an approximate idea of the numerics for $N_{g}=4$ as compared to $N_{g}=3$, we take $\alpha_{e m}^{-1}\left(m_{Z}\right)=128, \sin ^{2} \theta_{W}=$ .2316 and $\alpha_{3}\left(m_{Z}\right)=0.132$ at $t=t_{Z}$ and $\alpha\left(t_{U}\right)=0.0917$ (0.043) for $N_{g}=4$ $\left(N_{g}=3\right)$. This gives:

$$
\left(\begin{array}{l}
M_{1}\left(t_{Z}\right) \\
M_{2}\left(t_{Z}\right) \\
M_{3}\left(t_{Z}\right)
\end{array}\right) \stackrel{N_{g}=4}{=}\left(\begin{array}{c}
0.185 \\
0.37 \\
1.44
\end{array}\right) m_{1 / 2} ; \quad\left(\begin{array}{l}
M_{1}\left(t_{Z}\right) \\
M_{2}\left(t_{Z}\right) \\
M_{3}\left(t_{Z}\right)
\end{array}\right) \stackrel{N_{g}=3}{=}\left(\begin{array}{c}
0.39 \\
0.78 \\
3
\end{array}\right) m_{1 / 2} .
$$

These results are slightly modified when two-loop Yukawa contributions to the running of the gauge coupling couplings are incorporated. One finds coefficients for $N_{g}=4$ of roughly $0.194,0.386$ and 1.47 for $k=1,2$ and 3 , respectively. The main effect derives from the simple fact that $\alpha\left(t_{U}\right)$ decreases so that the $\alpha_{i}\left(t_{Z}\right) / \alpha\left(t_{U}\right)$ ratios increase. For example, for $m_{t^{\prime}}=m_{b^{\prime}}=100 \mathrm{GeV}, \alpha\left(t_{U}\right)$ is shifted down by about $5 \%$ to about 0.0874 . This results in an increase of the $N_{g}=4, k=1,2$ coefficients in Eq. (2) by about 5\%; for $k=3, \alpha_{3}\left(t_{Z}\right)$ also decreases (to about 0.1288 ) and the net Eq. (2) coefficient increase is only about $2 \%$.

We also recall that simple asymptotic results for the low-energy masses of the gauginos $\widetilde{\chi}_{i}^{0}$ and $\widetilde{\chi}_{i}^{+}$arise if $m_{W} \ll|| \mu\left| \pm M_{2}\right|$ (but not if $m_{W} \sim|| \mu\left| \pm M_{2}\right|$ ). These results are summarized in the chargino mass formulas and neutralino mass formulas given in Eqs. (36) and (37) (for charginos) and (40), (41), (42) and (43) (for neutralinos) of Ref. [29]. (See also Eqs. (5.3) and (6.8) of Ref. [28].) An example of such asymptotic results are the large $\mu$ relations $m_{\widetilde{\chi}_{1}^{0}} \sim M_{1}$ and $m_{\widetilde{\chi}_{1}^{+}} \sim$ $m_{\widetilde{\chi}_{2}^{0}} \sim M_{2}$, where $M_{1,2}$ are evaluated at energy scales of order $m_{Z}$. As seen from Figs. 13, 14 and 15, the above approximate mass formulae work reasonably well, but not perfectly, at large $m_{\widetilde{g}}$. For instance, at high $m_{\widetilde{g}}$ one finds $m_{\widetilde{\chi}_{1}^{0}} / m_{\tilde{g}} \sim 0.135$ compared to $M_{1} / M_{3} \sim 0.131$ (with $m_{t^{\prime}}=m_{b^{\prime}}=100 \mathrm{GeV}$ two-loop effects included in the gauge running).

One of the first priorities of a next linear $e^{+} e^{-}$collider (NLC) and the LHC will be to test the $M_{U}$-scale universality assumption for the $M_{i}$ 's. At the NLC it is estimated that $m_{\widetilde{\chi}_{1}^{+}}, m_{\widetilde{\chi}_{1}^{0}}$ and $m_{\widetilde{\chi}_{2}^{0}}$ can be measured to within a few $\mathrm{GeV}^{[30]}$ This 
will allow a pretty good determination of $M_{1}\left(t_{U}\right) / M_{2}\left(t_{U}\right)$. The optimal situation arises if $\mu$ is large, as is essentially always the case in three-generation models, and very often the case in the four-generation models. Then, as summarized above (and making the approximation that $m_{\widetilde{\chi}_{1}^{0}}$ and $m_{\widetilde{\chi}_{1}^{+}}$are of order $\left.m_{Z}\right) m_{\widetilde{\chi}_{1}^{0}} \sim M_{1}\left(t_{Z}\right)$ and $m_{\widetilde{\chi}_{1}^{+}} \sim m_{\widetilde{\chi}_{2}^{0}} \sim M_{2}\left(t_{Z}\right)$ and we have the more general result:

$$
\frac{M_{1}\left(t_{U}\right)}{M_{2}\left(t_{U}\right)}=\frac{\alpha_{2}\left(t_{Z}\right)}{\alpha_{1}\left(t_{Z}\right)} \frac{M_{1}\left(t_{Z}\right)}{M_{2}\left(t_{Z}\right)} \sim \frac{\alpha_{2}\left(t_{Z}\right)}{\alpha_{1}\left(t_{Z}\right)} \frac{m_{\widetilde{\chi}_{1}^{0}}}{m_{\tilde{\chi}_{1}^{+}}} .
$$

(For greater accuracy, one will wish to include the two-loop corrections to this relation when actually performing this test.) Our ability to test the universality for $i=3$ will probably be much more limited. At the LHC, $m_{\widetilde{g}}$ is unlikely to be measured to better than 50 to $100 \mathrm{GeV}$. Further, due to the small beta function at one-loop for $i=3$ in the case of four generations, two-loop corrections to the running of $\alpha_{3}$ can be significant, and these depend at least somewhat upon other model parameters. Nonetheless, it should be possible to extract a $\pm(20-30) \%$ value for $M_{2}\left(M_{U}\right) / M_{3}\left(M_{U}\right)$. The determination of the $M_{i}\left(t_{U}\right)$ is crucial, not only as a test of universality, but also in making predictions for the squark and slepton masses and testing $m_{0}$ universality.

But before turning to squarks and sleptons we must make a few more comments on the gaugino sector. First, the universality test will not have any significant sensitivity to $N_{g}=4$ vs. $N_{g}=3$. Second, although the absolute mass scales are sensitive to the limits on $m_{1 / 2}$ discussed earlier that keep $m_{1 / 2}$ from being as small for $N_{g}=4$ as it can be for $N_{g}=3$, it is difficult to use the absolute mass scale for a reliable probe of the value of $N_{g}$ since $N_{g}=3$ models with $N_{g}=4$ type $m_{\widetilde{g}}$ values are certainly entirely viable. Sum rules such as

$$
m_{\widetilde{\chi}_{1}^{+}} m_{\widetilde{\chi}_{2}^{+}}=\left|M_{2} \mu-m_{W}^{2} \sin 2 \beta\right|,
$$

following from the determinant of the mass matrix, are sensitive to the $N_{g}=4$ restriction $1 \lesssim \tan \beta \lesssim 3$ if $M_{2} \mu \sim m_{W}^{2}$. But such small values are not the norm, and to use this particular sum rule we must detect both $\widetilde{\chi}_{1}^{+}$and $\widetilde{\chi}_{2}^{+}$. Thus, we must include squark and slepton masses in our considerations in order to gain sensitivity to $N_{g}$.

We shall see below that the squark and slepton masses at one-loop are determined by the $m_{0}$ soft scalar mass (or masses should the $m_{0}$ values not be universal), by $\beta$ and by the functions:

$$
C_{k}(t) m_{1 / 2}^{2} \equiv\left(\begin{array}{c}
\frac{3}{5} \\
\frac{3}{4} \\
\frac{4}{3}
\end{array}\right) \times \frac{1}{\pi} \int_{t}^{t_{U}} d t g_{k}^{2}(t) M_{k}^{2}(t) .
$$

If one-loop evolution is used, then the integrals can be analytically carried out to 
give:

$$
C_{k}(t) m_{1 / 2}^{2}=2\left(\begin{array}{c}
\frac{3}{5} \\
\frac{3}{4} \\
\frac{4}{3}
\end{array}\right) M_{k}^{2}(t)\left[1-\frac{\alpha^{2}\left(t_{U}\right)}{\alpha_{k}^{2}(t)}\right] b_{k}^{-1} \quad \text { with } \quad b_{k}=\left(\begin{array}{c}
9-2 N_{g} \\
5-2 N_{g} \\
-\frac{3}{5}-2 N_{g}
\end{array}\right)
$$

where the $b_{k}$ are the one-loop beta function coefficients for the gauge couplings: $d \alpha_{k} / d t=-b_{k} \alpha_{k}^{2}$. If we input the relations of Eq. (1), then (at one-loop) we find:

$$
C_{k}(t)=2\left(\begin{array}{c}
\frac{3}{5} \\
\frac{3}{4} \\
\frac{4}{3}
\end{array}\right)\left[\frac{\alpha_{k}^{2}(t)}{\alpha^{2}\left(t_{U}\right)}-1\right] b_{k}^{-1}
$$

However, for four generations, one-loop evolution for $\alpha_{3}$ is generally not an adequate approximation when calculating $C_{3}$; the evolution of $\alpha_{3}$ is sensitive to twoloop terms, due to the small value of $b_{3}$, and $\alpha_{3}$ appears to the third power in the expression for $C_{3}$ in Eq. (5). The values of $C_{k}$ for $N_{g}=4$, computed numerically with full two-loop evolution for the couplings, are compared to those for $N_{g}=3$ in Table 1 at scales $m_{Z}$ and $1 \mathrm{TeV}$. For the $N_{g}=4$ computations we adopted $m_{t^{\prime}}=m_{b^{\prime}}=90 \mathrm{GeV}, m_{\tau^{\prime}}=m_{\nu^{\prime}}=50 \mathrm{GeV}$, and $\tan \beta=1.5$. Results are fairly insensitive to these choices. In the case of $N_{g}=4$, the results for $C_{k}$ would have been roughly $20 \%$ bigger had we neglected two-loop Yukawa terms in $\alpha_{3}$, which keep $\alpha_{3}$ somewhat smaller (as described earlier) than otherwise.

Table 1: We tabulate the $C_{k}$ values for $N_{g}=4$ and $N_{g}=3$ at the $m_{Z}$ and $1 \mathrm{TeV}$ energy scales. For $N_{g}=4$, we employ $m_{t^{\prime}}=m_{b^{\prime}}=90 \mathrm{GeV}, m_{\tau^{\prime}}=m_{\nu^{\prime}}=50 \mathrm{GeV}$, and $\tan \beta=1.5$.

\begin{tabular}{r|c|c|c|c}
\hline \hline$k$ & $C_{k}\left(t_{Z}\right) ; N_{g}=4$ & $C_{k}\left(t_{1 \mathrm{TeV}}\right) ; N_{g}=4$ & $C_{k}\left(t_{Z}\right) ; N_{g}=3$ & $C_{k}\left(t_{1 \mathrm{TeV}}\right) ; N_{g}=3$ \\
\hline 1 & 0.131 & 0.130 & 0.151 & 0.148 \\
2 & 0.376 & 0.371 & 0.484 & 0.459 \\
3 & 4.59 & 4.06 & 7.30 & 5.40 \\
\hline \hline
\end{tabular}

We see a useful feature of the $N_{g}=4$ results in Table 1: the $C_{k}(t)$ are much more independent of the low-energy scale choice than in the $N_{g}=3$ case. This can be understood from Eq. (7). If we make a change in $\alpha_{k}(t)$, then one can easily compute that:

$$
\frac{\Delta C_{k}(t)}{C_{k}(t)} \sim \frac{2 \Delta \alpha_{k}(t) \alpha_{k}(t) \alpha^{-2}\left(t_{U}\right)}{\left[\frac{\alpha_{k}^{2}(t)}{\alpha^{2}\left(t_{U}\right)}-1\right]} .
$$

First, consider $k=1,2$. Referring back to Fig. 1, we see that to a reasonable approximation $\Delta \alpha_{1,2}$ for $N_{g}=3$ and $N_{g}=4$ are very similar in magnitude in going 
from $m_{Z}$ to $1 \mathrm{TeV}$. Also from Fig. 1, we see that for $k=1,2$ the denominator of Eq. (8) is very substantially smaller, while $\alpha_{k}\left(m_{Z}\right) / \alpha\left(t_{U}\right)$ is much larger, for $N_{g}=3$ compared to $N_{g}=4$. In combination, these two effects lead to negligible change in $C_{1,2}$ in going from $m_{Z}$ to $1 \mathrm{TeV}$ when $N_{g}=4$, compared to a modest change for $N_{g}=3$. For $k=3$, the denominator is somewhat larger for $N_{g}=3$ than $N_{g}=4$, but the numerator is very much smaller for $N_{g}=4$, due not only to the decrease of $\alpha\left(t_{U}\right)^{-2}$ by a factor of $\sim 4$, but also because $\Delta \alpha_{3}$ (in going from $m_{Z}$ to $1 \mathrm{TeV}$ ) is very much smaller for $N_{g}=4$ than for $N_{g}=3$ (see Fig. 1). A substantial change in $C_{3}$ for $N_{g}=3$ is turned into a small change in the $N_{g}=4$ case. This is amusing both in its own right, but is particularly important for squark masses in that the small scale-sensitivity of $C_{3}$ means the exact low-energy scale down to which we evolve will have a weak impact upon the squark masses. In the case of $N_{g}=3$, when determining the running mass of a heavy squark, it is crucial to evaluate $C_{3}$ at the squark mass scale; for a heavy squark $C_{3}\left(t_{\widetilde{q}}\right)$ can be very substantially smaller than the $m_{Z}$-scale value as seen in Table 1 . In contrast, even for $N_{g}=3, C_{1,2}$ are much less sensitive to a change in scale.

Table 2: We tabulate the $f_{i k}$ and $D_{i}$ values for squarks and sleptons. Also given are the $m_{Z^{-}}$ scale $\hat{C}_{i}$ values for both $N_{g}=3$ and $N_{g}=4$. For $N_{g}=4$, we employ $m_{t^{\prime}}=m_{b^{\prime}}=100 \mathrm{GeV}$, $m_{\tau^{\prime}}=m_{\nu^{\prime}}=50 \mathrm{GeV}$, and $\tan \beta=1.5$. We use the notation $x_{W} \equiv \sin ^{2} \theta_{W}$.

\begin{tabular}{cc|cc|c|c|c}
\hline \hline & \multicolumn{2}{|c|}{$f_{i k}$} & $D_{i}$ & $\hat{C}_{i}\left(N_{g}=4\right)$ & $\hat{C}_{i}\left(N_{g}=3\right)$ \\
\cline { 2 - 6 }$i \backslash k$ & 1 & 2 & 3 & & & \\
\hline$\widetilde{\nu}$ & $\frac{1}{4}$ & 1 & 0 & $\frac{1}{2}$ & 0.41 & 0.52 \\
$\widetilde{\ell}_{R}$ & 1 & 0 & 0 & $-x_{W}$ & 0.13 & 0.15 \\
$\widetilde{\ell}_{L}$ & $\frac{1}{4}$ & 1 & 0 & $-\frac{1}{2}+x_{W}$ & 0.41 & 0.52 \\
$\widetilde{d}_{R}$ & $\frac{1}{9}$ & 0 & 1 & $-\frac{1}{3} x_{W}$ & 4.43 & 7.32 \\
$\widetilde{u}_{R}$ & $\frac{4}{9}$ & 0 & 1 & $\frac{2}{3} x_{W}$ & 4.48 & 7.37 \\
$\widetilde{d}_{L}$ & $\frac{1}{36}$ & 1 & 1 & $-\frac{1}{2}+\frac{1}{3} x_{W}$ & 4.80 & 7.79 \\
$\widetilde{u}_{L}$ & $\frac{1}{36}$ & 1 & 1 & $\frac{1}{2}-\frac{2}{3} x_{W}$ & 4.80 & 7.79 \\
\hline \hline
\end{tabular}

Let us now return to the squark and slepton masses. The primary sensitivity of these masses to $N_{g}$ is through the $C_{k}$ and the $N_{g}=4$ constraints on $m_{0}$ and $m_{1 / 2}$. To see this let us recall that, in terms of the $C_{k}$, one finds

$$
m_{i}^{2}=m_{0}^{2}+\hat{C}_{i} m_{1 / 2}^{2}+D_{i} m_{Z}^{2} \cos 2 \beta, \text { where } \quad \hat{C}_{i} \equiv \sum_{k} f_{i k} C_{k}
$$

The $f_{i k}$ and $D_{i}$ are tabulated in Table 2, as are $t_{Z}$-scale values for the $\hat{C}_{i}$, for both $N_{g}=3$ and $N_{g}=4$; in evaluating the $\hat{C}_{i}$ 's we have employed full two-loop evolution for all of the $\alpha_{k}$ 's in determining the $C_{k}$ 's, as noted earlier. Also noted 


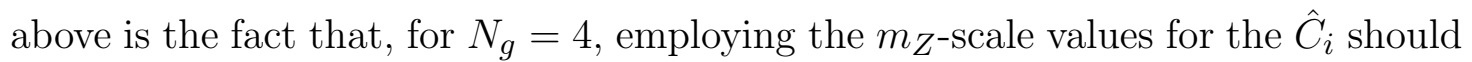
be a reasonably good approximation, even in the case of heavy squarks, due to the modest sensitivity of the $C_{k}$ coefficients to the energy scale. Of course, we must keep in mind that the numbers given above have presumed the universal value $M_{i}=m_{1 / 2}$ at $M_{U}$. Clearly, if the $M_{i}$ do not have a common $M_{U}$-scale value, one would have to redo all the computations.

What about the value of $\beta=\tan ^{-1} v_{2} / v_{1}$ ? Whether or not the slepton and squark masses are measurably sensitive to $\beta$ depends upon the relative size of the $m_{0}^{2}$ and $\hat{C}_{i} m_{1 / 2}^{2}$ terms compared to $m_{Z}^{2} \cos 2 \beta$. Certainly it would be nice to have sensitivity, since then it could be determined if $\tan \beta$ falls in the $1 \lesssim \tan \beta \lesssim$ 3 domain required if $N_{g}=4$. There are two mass-squared differences that, in principle, allow a direct determination of $\cos 2 \beta$ independent of whether the $m_{0}$ 's are universal and independent of any knowledge of the numerical values of the $\hat{C}_{i}$ :

$$
m_{\widetilde{\ell}_{L}}^{2}-m_{\widetilde{\nu}}^{2}=m_{\widetilde{d}_{L}}^{2}-m_{\widetilde{u}_{L}}^{2}=-\cos 2 \beta m_{Z}^{2}\left(1-x_{W}\right)
$$

where we employ the notation $x_{W} \equiv \sin ^{2} \theta_{W}$. (Note that since $\tan \beta \geq 1, \cos 2 \beta$ is always negative and these mass differences are positive.) For such differences to be sensitive to $\cos 2 \beta$ at just the $1 \sigma$ level, one must be able to measure a typical $\widetilde{m}$ to accuracy $\Delta \widetilde{m} / \widetilde{m}<m_{W}^{2} / 2 \widetilde{m}^{2}$. For masses of $100,250,500,1000 \mathrm{GeV}$, this means $\Delta \widetilde{m} / \widetilde{m}<32 \%, 5 \%, 1.3 \%, 0.32 \%$; clearly the last two accuracies are at a very difficult level even for an $e^{+} e^{-}$or $\mu^{+} \mu^{-}$collider of adequate energy. Determination of $\cos 2 \beta$ at the $3 \sigma$ level of accuracy would require factor of three smaller errors than those listed above. To be more precise regarding the possibilities for measuring the mass differences in Eq. (10), we outline some relevant issues and possible techniques in Appendix B.

The conclusion from Appendix B is that it will be difficult to measure $m_{\widetilde{\ell}_{L}}$ and $m_{\widetilde{\nu}}$ to much better than about $5 \%$ accuracy. (Since the mass scales for $m_{\widetilde{d}_{L}}$ and $m_{\widetilde{u}_{L}}$ are even larger than for the sleptons, and errors in the determination of their masses will be larger also (see Appendix B), it seems clear that it is the slepton sector upon which we should focus.) Given the $5 \%$ accuracy estimate for $m_{\tilde{\ell}_{L}}$ and $m_{\widetilde{\nu}}$ determination and comparing to the criteria of the previous paragraph, we conclude that a direct determination of $\cos 2 \beta$ will be problematical, especially if the slepton mass scales are as large as predicted for $N_{g}=4$. The ability to determine $\tan \beta$ could be even worse if $\tan \beta$ is large, since $\cos 2 \beta$ varies slowly with $\beta$ once $\beta$ approaches $\pi / 2$. Of course, for $N_{g}=4$ it is true that $\tan \beta$ is in the $1 \lesssim \tan \beta \lesssim 3$ range where our ability to extract $\tan \beta$ from a $\cos 2 \beta$ measurement would be maximal. Nonetheless, for the moment we must conclude that experimental determination of $\cos 2 \beta$ and thence $\tan \beta$ will be difficult unless the slepton masses are well below $200 \mathrm{GeV}$, as is possible only if $N_{g}=3$. Of course, further study is undoubtedly warranted and could reverse this conclusion. Fortunately, the uncertainty in $\cos 2 \beta$ is not the limiting factor in our ability to test the other crucial mass sum rules and relations discussed below. Other experimental and theoretical uncertainties are much more important in determining the limitations. 
$\underline{\text { Sum rules that do not mix slepton/squark and gaugino masses }}$

We can imagine two basic outcomes for our experimental attempts to determine $\cos 2 \beta$ : i) we are successful in getting at least a rough determination of $m_{Z}^{2} \cos 2 \beta$; or ii) our errors are too large to be sensitive to this kind of term. In either case, one would proceed next to a series of additional sum rules and mass relations that would provide tests of universality and the general unification/RGE framework and/or tests for $N_{g}=4 \mathrm{vs}$. $N_{g}=3$. For the moment, let us assume that both squarks and sleptons are observable, if not at an $e^{+} e^{-}$collider then perhaps at a $\mu^{+} \mu^{-}$collider of very high energy. ${ }^{[31]}$ If not, one must proceed quite differently; we will return to this alternate case in Sec. 6c.

To check $m_{0}$ universality independently of $N_{g}$, one must consider mass combinations that are independent of the $C_{i}$. The only simple example is

$$
\begin{aligned}
& 2\left(m_{\widetilde{u}_{R}}^{2}-m_{\widetilde{d}_{R}}^{2}\right)+\left(m_{\widetilde{d}_{R}}^{2}-m_{\widetilde{d}_{L}}^{2}\right)+\left(m_{\widetilde{\ell}_{L}}^{2}-m_{\widetilde{\ell}_{R}}^{2}\right) \\
& =\frac{10}{3} x_{W} m_{Z}^{2} \cos 2 \beta=\frac{10}{3} x_{W}\left(1-x_{W}\right)^{-1}\left(m_{\widetilde{\nu}}^{2}-m_{\widetilde{\ell}_{L}}^{2}\right) .
\end{aligned}
$$

This relation would be violated if all the $m_{0}$ 's appearing in Eq. (9) are not the same. Because of the large number of mass differences appearing above, to detect a violation of universality in just one of these differences at the $\Delta m_{0} / m_{0}=f$ level could require that the squark and slepton masses be measured with an accuracy that is $\lesssim f / 4$. From the discussion of Appendix B, we conclude that it will be difficult to obtain accuracy $f$ that is much better than $15-20 \%$. However, this level of accuracy is acceptable in the sense that we will find that tests of all other sum rules are more or less restricted to this same rough level of accuracy even if the $m_{0}$ were universal. This means that the limited accuracy of the test of $m_{0}$ universality will not dominate our ability to check various mass relations. Let us presume that universality is shown to be satisfied within the accuracy of measurement achievable. Then we can proceed to extract $m_{0}$ and test for $N_{g}=3 \mathrm{vs} . N_{g}=4$ to about this same level of accuracy.

There are various mass combinations that can be used to extract a universal $m_{0}$. However, we should note that the four quantities $m_{0}, C_{1}, C_{2}$, and $m_{Z}^{2} \cos 2 \beta$ cannot be extracted using just the three slepton masses - we must have some squark-sector measurements. One simple combination is

$$
m_{0}^{2}=m_{\widetilde{\ell}_{R}}^{2}-3\left(m_{\widetilde{u}_{R}}^{2}-m_{\widetilde{d}_{R}}^{2}\right)+4 x_{W}\left(1-x_{W}\right)^{-1}\left(m_{\widetilde{\nu}}^{2}-m_{\widetilde{\ell}_{L}}^{2}\right) .
$$

Note that the $C_{3}$ terms that cancel between $m_{\widetilde{u}_{R}}^{2}$ and $m_{\widetilde{d}_{R}}^{2}$ will be evaluated at very similar mass scales, so that the cancellation should be quite precise. The $C_{1}$ terms that cancel between $m_{\widetilde{\ell}_{R}}^{2}$ and $-3\left(m_{\widetilde{u}_{R}}^{2}-m_{\widetilde{d}_{R}}^{2}\right)$ could in principle be evaluated at 
somewhat different mass scales. As discussed previously, this would not matter in the $N_{g}=4$ case, but would lead to a small uncertainty for $N_{g}=3$; however, this uncertainty is much less than that from simple experimental errors in determining $m_{\widetilde{u}_{R}}^{2}-m_{\widetilde{d}_{R}}^{2}$. Indeed, if $m_{0}$ is small, then experimental errors in evaluating the right-hand side above could become a severe problem. We will not dwell further on this issue here.

The primary sensitivity to $N_{g}=4$ vs. $N_{g}=3$ derives from the fact that the gauge coupling at unification, $\alpha\left(t_{U}\right)$, is approximately twice as large when $N_{g}=4$ as it is when $N_{g}=3$. This leads to the possibly large difference between the $N_{g}=4$ and $N_{g}=3$ values of $C_{3}$, depending upon the energy scale of evaluation, illustrated in Table 1. However, it is not straightforward to exploit this sensitivity through mass relations involving squarks and sleptons alone, that is relations designed to eliminate direct reference to $m_{1 / 2}$. This is already apparent from the modest changes in the $C_{k}$ and $\hat{C}_{i}$ (see Tables 1 and 2) in going from 3 to 4 generations. The very best that one can do is to consider ratios such as $C_{3} / C_{1}$. As seen from Table 1, if squarks are at a low mass scale (of order $m_{Z}$ ) then this ratio takes the values 33.5 for $N_{g}=4$ as compared to 48.3 for $N_{g}=3$; but if squarks have masses of order $1 \mathrm{TeV}$, then we compute $C_{3} / C_{1}=36.5$, not very different from the 33.5 value for $N_{g}=4$. Experimental extraction of $C_{1}$ and $C_{3}$ can be done in a number of ways. An example is:

$$
\begin{aligned}
& C_{1} m_{1 / 2}^{2}=3\left(m_{\widetilde{u}_{R}}^{2}-m_{\widetilde{d}_{R}}^{2}\right)-3 x_{W}\left(1-x_{W}\right)^{-1}\left(m_{\widetilde{\nu}}^{2}-m_{\widetilde{\ell}_{L}}^{2}\right) \\
& C_{3} m_{1 / 2}^{2}=\left(m_{\widetilde{u}_{L}}^{2}-m_{\widetilde{\nu}}^{2}\right)+\frac{2}{3}\left(m_{\widetilde{u}_{R}}^{2}-m_{\widetilde{d}_{R}}^{2}\right),
\end{aligned}
$$

where the $C_{1} m_{1 / 2}^{2}$ result was already used in Eq. (12). Accuracy of about $10 \%$ or better would be very desirable for the experimental determination of $C_{1} m_{1 / 2}^{2}$ and $C_{3} m_{1 / 2}^{2}$ if we are to have a good chance of distinguishing $N_{g}=4$ from $N_{g}=3$, but would require determination of the $m_{\widetilde{u}_{L}}-m_{\widetilde{\ell}_{L}}$ and $m_{\widetilde{u}_{R}}-m_{\widetilde{d}_{R}}$ mass differences to roughly $3 \%$, a challenging task even at an $e^{+} e^{-}$collider, as we have already discussed.

Some mass sum rules are quite insensitive to $N_{g}=3$ vs. $N_{g}=4$. Such mass relations can be used to test the general RGE approach, somewhat independently of the generation issue. To illustrate, consider the sample relation (3.19) in Ref. [28]:

$$
\begin{aligned}
m_{\widetilde{e}_{L}}^{2}-m_{\widetilde{e}_{R}}^{2} & =\frac{\left(C_{2}-\frac{3}{4} C_{1}\right)}{\left(C_{3}-\frac{8}{9} C_{1}\right)}\left(m_{\widetilde{d}_{R}}^{2}-m_{\widetilde{e}_{R}}^{2}\right) \\
& +\cos 2 \beta m_{Z}^{2}\left[-\frac{2}{3} x_{W} \frac{\left(C_{2}-\frac{3}{4} C_{1}\right)}{\left(C_{3}-\frac{8}{9} C_{1}\right)}-\left(\frac{1}{2}-2 x_{W}\right)\right] .
\end{aligned}
$$

* We have corrected a sign error in this equation. 
We note that $\cos 2 \beta<0$ (for $\tan \beta>1$ ) so that the sign of the second term is opposite to the sign of the factor in brackets. Adopting our results from Table 1 for the $C_{k}$, we find at scale $m_{Z}$ :

$m_{\widetilde{e}_{L}}^{2}-m_{\widetilde{e}_{R}}^{2}=(0.052$ or 0.062$)\left(m_{\widetilde{d}_{R}}^{2}-m_{\widetilde{e}_{R}}^{2}\right)-\left[(19.3 \mathrm{GeV})^{2}\right.$ or $\left.(19.6 \mathrm{GeV})^{2}\right] \cos 2 \beta$

for $N_{g}=3$ or $N_{g}=4$, respectively. Since this relation assumes universality among the $m_{0}$, the relatively minor changes in the coefficients for $N_{g}=4$ compared to $N_{g}=3$ implies greater sensitivity to possible universality violation among the $m_{0}$ than to $N_{g}$. We note that for accurate examination of this sum rule, an accurate value for $C_{3}$ is absolutely necessary. As noted above, in computing $C_{3}$ it is critical when $N_{g}=4$ to include the two-loop contributions to the running of $\alpha_{3}$ given that the one-loop beta function is anomalously small, being proportional to $9-2 N_{g}$.

Third-generation mass sum rules (4.5) and (4.10) of Ref. [28], which are independent of the $m_{0}$ universality assumption, are unaffected by a fourth family. However, since they require that the $\widetilde{b}$ mass matrix is diagonal to a good approximation (which is generally true for the third family since $m_{b}$ is small), the analogous sum rules do not apply for the fourth family due to the large value of $m_{b^{\prime}}$.

A generalization of the sum rule (4.6) of Ref. [28] for the splitting $m_{\widetilde{t}_{1}}^{2}-m_{\widetilde{t}_{2}}^{2}$ can be found in the four-family case. It is not especially useful, however, since it relates $m_{\widetilde{t}_{1}^{\prime}}^{2}-m_{\widetilde{t}_{2}^{\prime}}^{2}$ to $m_{\widetilde{b}_{1}^{\prime}}^{2}-m_{\widetilde{b}_{2}^{\prime}}^{2}$ via $A_{t^{\prime}}$ and $A_{b^{\prime}}$ dependent terms; thus, we do not display it here.

$\underline{\text { Sum rules that mix squark/slepton masses and gaugino masses }}$

So far, we discussed only mass relations and sum rules that referred either to squark/slepton masses only, or to gaugino masses only. However, it should be clear from all our previous discussions that the real sensitivity to $N_{g}=4 v s$. $N_{g}=3$ lies in the dramatic shift of slepton/squark masses at given $m_{\tilde{g}}$. This was already discussed in Sec. 6a, and is further evident from the relations of Eq. (13), once the substitution $m_{1 / 2}=m_{\tilde{g}} \alpha\left(t_{U}\right) / \alpha_{3}\left(m_{\tilde{g}}\right)$ is made and it is recalled that $\left.\alpha\left(t_{U}\right)\right|_{N_{g}=4}=0.092$ compared to $\left.\alpha\left(t_{U}\right)\right|_{N_{g}=3}=0.043$. However, because of inherent inaccuracies in experimentally measuring $m_{\widetilde{g}}$ it is better to reference $M_{1}\left(t_{Z}\right)$ or $M_{2}\left(t_{Z}\right)$, see Eq. (2), which can be quite well determined once the experimentally more accessible masses $m_{\widetilde{\chi}_{1}^{0}}$ and $m_{\widetilde{\chi}_{1}^{+}}$are measured. A particularly simple example is provided by $m_{\widetilde{d}_{R}}^{2}-m_{\widetilde{\ell}_{R}}^{2}$. From Table 2 we find that (using $m_{Z}$-scale $\hat{C}_{i}$ values for $1 \mathrm{TeV}$ scale values the difference in coefficients is even greater) and the relations 
of Eq. (2),

$$
\begin{aligned}
m_{\widetilde{d}_{R}}^{2}-m_{\widetilde{\ell}_{R}}^{2} & =\delta m_{0}^{2}+(4.3 \text { or } 7.17) m_{1 / 2}^{2}+\frac{2}{3} x_{W} m_{Z}^{2} \cos 2 \beta \\
& =\delta m_{0}^{2}+(31.5 \text { or } 11.8) M_{2}^{2}\left(t_{Z}\right)+\frac{2}{3} x_{W} m_{Z}^{2} \cos 2 \beta,
\end{aligned}
$$

for $N_{g}=4$ or $N_{g}=3$, respectively. Here, we have included a possible violation of $m_{0}$ universality, $\delta m_{0}^{2} \equiv m_{0}^{2}\left(\widetilde{d}_{R}\right)-m_{0}^{2}\left(\widetilde{\ell}_{R}\right)$. Experimental uncertainties in determining the coefficient, $C_{M_{2}}$, of $M_{2}^{2}\left(t_{Z}\right)$ will be dominated by experimental errors in determining $m_{\widetilde{d}_{R}}^{2}-m_{\widetilde{\ell}_{R}}^{2}$ and $\delta m_{0}^{2}$. The latter error, as discussed with regard to Eq. (11), is expected to be several times as large as the former. Very crudely, for experimental uncertainties in the squark/slepton mass measurements of size $\Delta \widetilde{m} / \widetilde{m} \sim f$, one expects an experimental uncertainty in the $M_{2}^{2}$ coefficient of order $\Delta C_{M_{2}} \sim 3-5 \times f \widetilde{m}^{2} / M_{2}^{2}$. We have already seen that $f$ is very unlikely to be better than $f \sim 0.1$. Taking $\widetilde{m} \sim 1 \mathrm{TeV}$ and $M_{2} \sim 200 \mathrm{GeV}$ implies a $\Delta C_{M_{2}} \sim 7.5-12.5$. This is smaller than the predicted $N_{g}=4$ vs. $N_{g}=3$ difference, suggesting we would, in fact, be sensitive to $N_{g}$ by measuring masses of the gauginos and the squarks and sleptons of just the first two generations.

\section{6c. Non-Squark Spectrum Tests for Universality and Four Generations}

As repeatedly noted, mass determinations will be most easily performed at an $e^{+} e^{-}$collider of appropriate energy. Regardless of the value of $N_{g}$, chargino pair production is very likely to be within reach of a $\sqrt{s}=500 \mathrm{GeV} \mathrm{NLC}$. In the case of $N_{g}=3$, slepton pair production is also very likely to be possible for $\sqrt{s}=500 \mathrm{GeV}$ if boundary conditions of the no-scale or dilaton type are appropriate. For $N_{g}=4$, however, these same boundary conditions predict much larger slepton masses, and a higher energy machine would probably be required. However, for either $N_{g}=3$ or $N_{g}=4$, the squarks are much heavier still, and it is quite possible that an $e^{+} e^{-}$ or $\mu^{+} \mu^{-}$collider would be built that could produce the neutralinos, charginos and sleptons, but not the gluino and squarks. Thus, it is desirable to consider what kinds of tests of universality, the RGE framework, and $N_{g}$ can be performed without reference to either the gluino or the squarks. In particular, we wish to determine if there is a test for $N_{g}=3$ vs. $N_{g}=4$ that can be performed using only the $\widetilde{\chi}_{1}^{0}, \widetilde{\chi}_{1}^{+}$masses and the masses of the first or second family sleptons, which are those most likely to be both easily accessible and most precisely predictable (i.e. without reference to $A$ parameters and $L-R$ mixing). So, let us imagine that the $M_{i}$ for $i=1,2$ have been determined with reasonable accuracy from the observed values of $m_{\widetilde{\chi}_{1}^{0}}$ and $m_{\widetilde{\chi}_{1}^{+}}$, following the procedure described earlier. If we then assume a value of $N_{g}, m_{1 / 2}$ will also be computable from Eq. (2). Finally, the $C_{i}$ can also be computed for the assumed value of $N_{g}$. 
As mentioned earlier, the $\tilde{\ell}_{L}$ and $\tilde{\ell}_{R}$ masses are very sensitive to $N_{g}$ when expressed in terms of the parameter $\xi_{0}$. In what follows, we keep track of a possible difference between the $\xi_{0}$ values in the $L$ and $R$ sectors, i.e. of possible universality violation. We have, from Table 2 ,

$$
\begin{aligned}
& m_{\widetilde{\ell}_{L}}^{2} \sim\left[\xi_{0}^{L}\right]^{2} m_{1 / 2}^{2}+(0.41 \text { or } 0.52) m_{1 / 2}^{2}+\left(-\frac{1}{2}+x_{W}\right) m_{Z}^{2} \cos 2 \beta, \\
& m_{\widetilde{\ell}_{R}}^{2} \sim\left[\xi_{0}^{R}\right]^{2} m_{1 / 2}^{2}+(0.13 \text { or } 0.15) m_{1 / 2}^{2}-x_{W} m_{Z}^{2} \cos 2 \beta,
\end{aligned}
$$

for $N_{g}=4$ or $N_{g}=3$, respectively. We note that the $\hat{C}_{i}$ for $m_{\widetilde{\ell}_{L}}$ and $m_{\widetilde{\ell}_{R}}$ are quite different, but not terribly sensitive to $N_{g}=3$ vs. $N_{g}=4$. However, by converting to the value of, for example, $M_{1}\left(t_{Z}\right)$ using Eq. (2), a large difference between the $N_{g}=4$ and $N_{g}=3$ phenomenology emerges. In the approximation that $m_{\widetilde{\chi}_{1}^{0}} \sim M_{1}\left(t_{Z}\right)$ we have

$$
\begin{aligned}
r_{\widetilde{\ell}_{L}}^{2} & \equiv \frac{m_{\widetilde{\ell}_{L}}^{2}}{m_{\widetilde{\chi}_{1}^{0}}^{2}} \sim(29 \text { or } 7)\left[\xi_{0}^{L}\right]^{2}+(12 \text { or } 3)+\left(-\frac{1}{2}+x_{W}\right) \frac{m_{Z}^{2}}{m_{\widetilde{\chi}_{1}^{0}}^{2}} \cos 2 \beta, \\
r_{\widetilde{\ell}_{R}}^{2} & \equiv \frac{m_{\widetilde{\ell}_{R}}^{2}}{m_{\widetilde{\chi}_{1}^{0}}^{2}} \sim(29 \text { or } 7)\left[\xi_{0}^{R}\right]^{2}+(4 \text { or } 1)-x_{W} \frac{m_{Z}^{2}}{m_{\widetilde{\chi}_{1}^{0}}^{2}} \cos 2 \beta,
\end{aligned}
$$

for $N_{g}=4$ or $N_{g}=3$, respectively.

We plot our precise numerical results for $r_{\widetilde{\ell}_{L}}$ and $r_{\widetilde{\ell}_{R}}$ as a function of $\xi_{0}$ in Fig. 16. We have taken $m_{t}=165 \mathrm{GeV}$ and $\tan \beta=1.5$. In the case of $N_{g}=4$, we choose $m_{t^{\prime}}=m_{b^{\prime}}=100 \mathrm{GeV}$ and $m_{\tau^{\prime}}=m_{\nu^{\prime}}=50 \mathrm{GeV}$. (All masses are running masses in this discussion.) The graph is given for the specific value of $m_{\widetilde{\chi}_{1}^{0}}=117 \mathrm{GeV}$. For $N_{g}=4(3)$ this corresponds to $m_{1 / 2} \sim 600 \mathrm{GeV}(\sim 300 \mathrm{GeV})$, respectively. This kind of plot has a number of important advantages. First, there is no sensitivity to the $A$ parameter for the first and second generation masses being considered. Second, $\tan \beta$ enters into the location of the $\xi_{0}=0$ intercepts, but only weakly if $m_{\widetilde{\chi}_{1}^{0}} \sim m_{Z}$. Third, by measuring the $\widetilde{\ell}_{L}$ and $\widetilde{\ell}_{R}$ masses in units of $m_{\widetilde{\chi}_{1}^{0}}$, the plot is quite independent of the actual value of $m_{1 / 2}$ (or, equivalently, $\left.m_{\widetilde{\chi}_{1}^{0}}\right)$, as seen in the approximate relations, Eq. (18).

Fig. 16 exhibits a rather big difference for the functional dependence of $r_{\widetilde{\ell}_{L}}$ and $r_{\widetilde{\ell}_{R}}$ on $\xi_{0}$, depending on $N_{g}$. Suppose that experiment yields $r_{\widetilde{\ell}_{R}}=4.5$. For $N_{g}=3$ this implies $\xi_{0}^{R} \sim 1.7$ and, if $\xi_{0}^{L}=\xi_{0}^{R}$, i.e. the $\xi_{0}$ are universal, $r_{\widetilde{\ell}_{L}} \sim 4.7$; for $N_{g}=4$ we find $\xi_{0}^{R} \sim 0.8$ and $r_{\widetilde{\ell}_{L}} \sim 5.3$. Since $m_{\widetilde{\chi}_{1}^{0}} \sim 117 \mathrm{GeV}$ in this case, this corresponds to $m_{\widetilde{\ell}_{L}}\left(N_{g}=4\right)-m_{\tilde{\ell}_{L}}\left(N_{g}=3\right) \sim 70 \mathrm{GeV}$ compared to perhaps 
$10-20 \mathrm{GeV}$ uncertainty in the $\widetilde{\ell}_{L}$ mass measurement. Of course, to measure $m_{\widetilde{\ell}_{L}}$ and $m_{\widetilde{\ell}_{R}}$ in the mass range above $400 \mathrm{GeV}$, required in this example, demands a very high energy $e^{+} e^{-}$or $\mu^{+} \mu^{-}$collider.

As one moves to lower $\xi_{0}$ values, the discriminating power of this procedure slowly increases. Suppose the experimentally measured value is $r_{\widetilde{\ell}_{R}}=3$. If $N_{g}=3$ this would imply $\xi_{0}^{R}=1.1$, and $\xi_{0}^{L}=\xi_{0}^{R}$ would then imply $r_{\widetilde{\ell}_{L}}=3.4$. In contrast, if $N_{g}=4, r_{\widetilde{\ell}_{R}}=3$ corresponds to $\xi_{0}^{R}=0.45$, yielding $r_{\widetilde{\ell}_{L}}=4.1$, or $m_{\widetilde{\ell}_{L}}\left(N_{g}=\right.$ 4) $-m_{\widetilde{\ell}_{L}}\left(N_{g}=3\right) \sim 82 \mathrm{GeV}$, a bit larger than our previous result.

How sensitive are we to a breaking of universality and the uncertainty in $\tan \beta$ ? Consider first the possibility that $\xi_{0}^{L}$ and $\xi_{0}^{R}$ are not the same. Returning to our first example, and neglecting effects of the $\operatorname{Tr}\left(\mathrm{Ym}^{2}\right)$ evolution terms, a measurement of $r_{\tilde{\ell}_{L}}=5.3$ in agreement with the $N_{g}=4$ prediction without universality violation could be reinterpreted as a prediction of $N_{g}=3$ provided $\xi_{0}^{L} \sim 1.9$, as compared to the value $\xi_{0}^{R}=1.7$ required for $r_{\widetilde{\ell}_{R}}=4.5$ for $N_{g}=3$. This translates to a breaking of universality in the amount:

$$
\frac{\xi_{0}^{L}-\xi_{0}^{R}}{\frac{1}{2}\left(\xi_{0}^{L}+\xi_{0}^{R}\right)} \sim \frac{0.2}{1.8} \sim 11 \%
$$

Thus, $\sim 10 \%$ universality violation could correct for the $N_{g}=4$ vs. $N_{g}=3$ difference. This is an intrinsic ambiguity when only slepton, chargino and neutralino masses are available.

In the above, we have purposely chosen situations where consistent solutions for both $N_{g}=3$ and $N_{g}=4$ are possible. It could easily happen that consistent choices for $\xi_{0}^{R}$ and $\xi_{0}^{L}$ are simply not possible for the observed values of $r_{\widetilde{\ell}_{R}}$ and/or $r_{\widetilde{\ell}_{L}}$. In particular, if $r_{\widetilde{\ell}_{R}} \lesssim 2.0$ or $r_{\widetilde{\ell}_{L}} \lesssim 3.5$ then $N_{g}=4$ is excluded, whereas $N_{g}=3$ models would be possible. In fact, if $N_{g}=3$ and $\xi_{0}$ is in the preferred $\xi_{0} \lesssim 1$ range, then $r_{\widetilde{\ell}_{L}}<3$ and $N_{g}=4$ would be immediately excluded. The lower bounds on $r_{\widetilde{\ell}_{L}}$ and $r_{\widetilde{\ell}_{R}}$ for $N_{g}=4$ are, of course, equivalent to lower bounds on $m_{\widetilde{\ell}_{L}}, m_{\widetilde{\ell}_{R}}$ of $410,234 \mathrm{GeV}$ for this particular $m_{\widetilde{\chi}_{1}^{0}}$ value. And, these lower bounds can only be reached if one is willing to accept the no-scale $m_{0}=0$ boundary conditions. In another extreme, it could happen that one measures $r_{\widetilde{\ell}_{R}} \sim r_{\widetilde{\ell}_{L}} \gtrsim 7-8$. Such large slepton masses relative to $m_{\widetilde{\chi}_{1}^{0}}$ require very large $\xi_{0}$ values if $N_{g}=3$, well beyond any acceptable model range. In contrast, if $N_{g}=4$ the required $\xi_{0}$ values are perfectly reasonable.

We note that sensitivity of these results to $\cos 2 \beta$ is really quite limited. For $m_{\widetilde{\chi}_{1}^{0}} \gtrsim m_{Z}$, and taking both $x_{W}$ and $-\frac{1}{2}+x_{W}$ to be of magnitude $\sim 0.25$, the full range of $\cos 2 \beta$ between 0 and -1 corresponds to $\Delta r \sim 0.125 / r \times m_{Z} / m_{\widetilde{\chi}_{1}^{0}} \lesssim$ 
$0.125 / r$. Since the $r$ values are typically at least of order 1 , most probably much larger, we see that the $\cos 2 \beta$ uncertainty is, at worst, roughly the same as the experimental error, and is not significant compared to the predicted $r$ differences or possible universality questions.

Finally, we note that even if we have an ambiguity in the decision between non-universality and $N_{g}=3$ vs. $N_{g}=4$, in general we will have a very rough determination of the general size of $\xi_{0}$. In the above example, we would at least know that the $\xi_{0}$ values were $\gtrsim 0.8$ if $N_{g}=4$ or $\gtrsim 1.7$ if $N_{g}=3$. This would allow us to set the overall boundary condition picture.

\section{Experiment and a Fourth Family}

In this section, unless otherwise indicated, all masses are pole masses. Where appropriate, running masses will be explicitly written in the $m(m)$ notation. Very roughly, pole masses are higher than running $m(m)$ masses by about 5 or $6 \%$, based on the dominant correction $m($ pole $)=m(m)\left[1+\frac{4}{3 \pi} \alpha_{s}(m)\right]$.

In all our discussions up to this point, we have adopted the conventional interpretation that the CDF and D0 events derive from $t \rightarrow b W$ decays, with $m_{t}$ (pole $) \sim 175 \mathrm{GeV}$. Alternative possibilities in which the events at CDF and D0 are reinterpreted as $m_{t^{\prime}} \gtrsim 175 \mathrm{GeV}$ events with $t^{\prime} \rightarrow b W$, while the $t$ is not currently observed, are not consistent within the perturbative MSSM GUT framework. In the first such option, the $t$ is not observed because it is too heavy, $m_{t}\left(m_{t}\right) \gg 165 \mathrm{GeV}$. The difficulty with this option is that for such large values of $m_{t}$ the maximum value of $m_{t^{\prime}}\left(m_{t^{\prime}}\right)$ allowed by perturbativity is much below $165 \mathrm{GeV}$ and the $t^{\prime} \bar{t}^{\prime}$ events would not have the same characteristics (e.g. reconstructed mass and cross section) as observed by CDF and D0. In the second such option (emphasized in Ref. [14]), the $t$ is much lighter than the $t^{\prime}, m_{t} \sim m_{W}$, but is not detected because its decays are dominated by $t \rightarrow \widetilde{t}_{1} \widetilde{\chi}_{1}^{0}$. However, in the MSSM GUT context, the latter decay does not occur if the soft-SUSY-breaking parameters are approximately universal, since (as we have seen) the $\widetilde{t}_{1}$ is always much heavier than the $t$. Further discussion of this latter case will appear in Sec. 7a. Certainly, the phenomenology of the $t^{\prime}$ and $b^{\prime}$ at the Tevatron that we present depends crucially on the $m_{t} \sim 175 \mathrm{GeV}>m_{b^{\prime}}, m_{t^{\prime}}$ hierarchy, not to mention the relative size of $m_{t^{\prime}} v s . m_{b^{\prime}}$, and on the mixing pattern between the generations.

In Sec. 7a we will discuss direct limits on the fourth family fermions coming from collider experiments, especially the CDF and D0 experiments at the Tevatron, assuming that $m_{t}($ pole $) \sim 175 \mathrm{GeV}$. We find that a relatively small portion of $m_{b^{\prime}}, m_{t^{\prime}}$ parameter space survives all such constraints for the more natural choices of CKM mixing between the fourth generation and lower generations. We also 
describe in more detail why the $m_{t} \sim m_{W}$ scenario is not viable for universal softSUSY-breaking boundary conditions. In Sec. $7 \mathrm{~b}$ we give the constraints on $m_{t^{\prime}}, m_{b^{\prime}}$ arising from consistency with precision electroweak data. Not surprisingly, these tend to rule out large $m_{t^{\prime}}$ compared to $m_{b^{\prime}}$. Finally, in Sec. $7 \mathrm{c}$ we speculate on deviations in single-jet inclusive and di-jet spectra that could arise as a result of a full four-family content at relatively low mass scales.

\section{7a. Direct Collider Searches}

As we have demonstrated, the requirement that all Yukawa couplings remain perturbative places rather strict upper bounds on the $t^{\prime}, b^{\prime}, \nu^{\prime}$ and $\tau^{\prime}$ masses, and the possibility of an MSSM fourth generation may well be settled within the next few years by searches (i) at the Tevatron, for an appropriate set of new signatures (i.e. besides $t \rightarrow b+W$ ), and (ii) at LEP-II, via detection of direct $\tau^{\prime}+\bar{\tau}^{\prime}$ and/or $\nu^{\prime}+\bar{\nu}^{\prime}$ production.

LEP has set the firmest direct limit, namely $m_{f} \gtrsim m_{Z} / 2$ for $f=b^{\prime}, t^{\prime}, \tau^{\prime}, \nu^{\prime}$. We have also seen that the value of $m_{t} \sim 175 \mathrm{GeV}$, as determined by the recent CDF and D0 results, ${ }^{[20]}$ is such as to imply $m_{t}>m_{b^{\prime}}, m_{t^{\prime}}$ for perturbative consistency.

Let us begin by noting the critical difference between the $m_{t^{\prime}}<m_{b^{\prime}}$ and $m_{t^{\prime}}>$ $m_{b^{\prime}}$ cases, assuming that $m_{t}>m_{t^{\prime}}, m_{b^{\prime}}$. If $m_{t^{\prime}}<m_{b^{\prime}}$, then the $t^{\prime} \rightarrow W b$ decay proceeds at tree-level at a rate determined by the CKM entry $V_{t^{\prime} b}$. One-loop processes, such $t^{\prime} \rightarrow c g, t^{\prime} \rightarrow c \gamma, t^{\prime} \rightarrow c Z^{(*)}$ and so forth occur at rates determined by $V_{t^{\prime} s} V_{c s}$ and $V_{t^{\prime} b^{\prime}} V_{c b^{\prime}}$ times a loop integration factor. It would be extremely unnatural for the product of a loop integration factor times a two-generationskipping CKM matrix element to exceed the one-generation-skipping CKM $V_{t^{\prime} b}$. Thus, it is almost certain that $t^{\prime} \rightarrow W b$ decays would be completely dominant if $m_{t^{\prime}}<m_{b^{\prime}}$. This situation should be contrasted with the reverse case of $m_{t^{\prime}}>$

$m_{b^{\prime}}$, for which the interesting issue is how the $b^{\prime}$ decays. The decay $b^{\prime} \rightarrow W c$ occurs at tree-level with rate determined by $V_{c b^{\prime}}$. The one-loop rates for $b^{\prime} \rightarrow b g$, $b^{\prime} \rightarrow b \gamma, b^{\prime} \rightarrow b Z^{(*)}$ and so forth are determined by $V_{t b^{\prime}} V_{t b}$ and $V_{t^{\prime} b^{\prime}} V_{t^{\prime} b}$ times loop integration factors. It is actually rather likely that the two-generation-skipping CKM element $V_{c b^{\prime}}$ is smaller than the one-generation-skipping $V_{t b^{\prime}}$ or $V_{t^{\prime} b}$ times the loop integration factor. In this case, the flavor changing decays $b^{\prime} \rightarrow b X$ would dominate over the two-generation skipping $b^{\prime} \rightarrow W c$ decay. The different decay structure for $m_{t^{\prime}}<m_{b^{\prime}}$ as compared to $m_{t^{\prime}}>m_{b^{\prime}}$ has crucial phenomenological implications.

To repeat, if $m_{t^{\prime}}<m_{b^{\prime}}$ it is almost certain that the $t^{\prime}$ will decay in a topquark-like manner to $W b$ (or $W^{*} b$, depending on $m_{t^{\prime}}$ ). Events of this type are clearly ruled out by early CDF and D0 'top-quark' limits for all $m_{t^{\prime}}<m_{b^{\prime}}$ regions allowed by the four-generation perturbativity constraints, see Fig. 2. Thus, we immediately eliminate roughly half of the perturbatively allowed regions. We now focus on experimental restrictions that arise if $m_{t^{\prime}}>m_{b^{\prime}}$.

$\underline{b^{\prime} \bar{b}^{\prime} \text { Production at the Tevatron }}$ 
If $m_{t^{\prime}}>m_{b^{\prime}}$, then in $b^{\prime} \bar{b}^{\prime}$ production at the Tevatron we must consider the two more or less competitive $b^{\prime}$ decay scenarios outlined above. If $V_{b^{\prime} c}$ (or, much less likely, $V_{b^{\prime} u}$ ) is sufficiently large that $b^{\prime} \rightarrow c W\left(\right.$ or $b^{\prime} \rightarrow u W$ ) decays are dominant, the published data ${ }^{[32]}$ already rule out $m_{b^{\prime}}<85 \mathrm{GeV}$ at $95 \%$ C.L. (Presumably this old result will soon be updated using the full CDF and D0 data sets.) But, if the $b^{\prime}$ is essentially unmixed with light generations, it will decay via flavor-changing neutral current (FCNC) channels: $b^{\prime} \rightarrow b \gamma, b g$ or $b Z^{*}$ for $m_{b^{\prime}}<m_{Z}$, with $b^{\prime} \rightarrow b Z$ becoming dominant for $m_{b^{\prime}}>m_{Z}+m_{b}$.

Present data would appear to rule out a significant rate for $b^{\prime} \rightarrow b Z$ decays by virtue of there not being room for an excess number of $Z$ 's beyond that predicted on the basis of the standard $q \bar{q}$ annihilation mechanism. Taking a $b^{\prime}$ mass of $100 \mathrm{GeV}$ (roughly the maximum possible if $m_{t^{\prime}}>m_{b^{\prime}}$ ), the cross section (without inclusion of any $K$ factor) at $\sqrt{s}=1.8 \mathrm{TeV}$ (Fermilab Tevatron) is about $60 \mathrm{pb}$. For an integrated luminosity of $67 \mathrm{pb}^{-1}$ this implies roughly $4000 \mathrm{~b}^{\prime} \bar{b}^{\prime}$ events. If we imagine looking for $Z$ 's from the decay of either (or both) $b^{\prime}$ 's, then this number is to be multiplied by $\left[1-B R\left(b^{\prime} \rightarrow \text { non }-Z\right)^{2}\right]$. Given the small phase space, $m_{b^{\prime}}-m_{Z}-m_{b} \sim 5 \mathrm{GeV}$ and the low value of $m_{t^{\prime}} \sim 100 \mathrm{GeV}$ required by our perturbative constraints (which impacts the one-loop Penguin diagram calculations), we expect ${ }^{[7]} B R\left(b^{\prime} \rightarrow\right.$ non $\left.-Z\right)$ to be significant but probably $\lesssim 0.7$, implying $\gtrsim 2000$ events in which at least one of the $b^{\prime}$ 's decays to a $Z$. Using $B R\left(Z \rightarrow \ell^{+} \ell^{-}\right)=0.067$, and an overall two-lepton cuts/acceptance/detection efficiency of about $\epsilon=0.5$, we find $\gtrsim 80$ events where at least one $Z$ appears in the $b^{\prime} \bar{b}^{\prime}$ final state and has a clearly detectable leptonic decay. (If we take $B R\left(b^{\prime} \rightarrow Z b\right)=1$, and adjust for luminosity, this number is consistent with the pre- $p_{T}(Z)$-cut numbers of Agrawal, Ellis and Hou, Ref. [7], for the sum of $\ell^{+} \ell^{-}+j j+X$ and $4 \ell+X$ event numbers at $m_{b^{\prime}}=100 \mathrm{GeV}$.) In addition, Fig. 2 shows that if $m_{t^{\prime}}$ is to be larger than $m_{b^{\prime}}$ and $m_{b^{\prime}} \sim 100 \mathrm{GeV}$, then $m_{t^{\prime}}$ can only be just larger than $m_{b^{\prime}}$. In this case, the $t^{\prime} \bar{t}^{\prime}$ production cross section would be more or less the same as the $b^{\prime} \bar{b}^{\prime}$ cross section, thereby almost doubling the 80 events, assuming that $t^{\prime} \rightarrow b^{\prime} W^{*}$ decay is dominant. Although a specific analysis has not been presented for the latest CDF or D0 data with regard to this point, we expect that such a large number of additional relatively clean $Z$ events can be ruled out. For an integrated luminosity of $L=150 \mathrm{pb}^{-1}$, it would seem that one could virtually eliminate the $m_{b^{\prime}}>m_{Z}+m_{b}$ case down to very near the threshold. However, if $b^{\prime} \rightarrow b h^{0}$ is also kinematically allowed, the number of extra $Z$ events would be much smaller and $m_{b^{\prime}}>m_{Z}+m_{b}$ would not be excluded. We shall return to this issue shortly.

Thus, consistency with experiment probably requires that $m_{b^{\prime}}<m_{Z}+m_{b}$, and that $b^{\prime}$ decays are dominated by the FCNC channels outlined above. These 'non-standard' decays would yield distinctly different signatures than the leptonplus-jet signatures of charged current decays that are already mostly excluded, and new search strategies are required. Perhaps the cleanest discovery channel makes use of $b^{\prime} \bar{b}^{\prime}$ production followed by double $b^{\prime} \rightarrow b \gamma$ decay. As an example consider $m_{b^{\prime}}=90 \mathrm{GeV}(95 \mathrm{GeV})$. The Tevatron cross section (without including 
any QCD $K$ factor enhancement) for $b^{\prime} \bar{b}^{\prime}$ production is $\sim 100 \mathrm{pb}(76 \mathrm{pb})$, corresponding to $\sim 6700(5100)$ events for $L=67 \mathrm{pb}^{-1}$. Assuming $B R\left(b^{\prime} \rightarrow b \gamma\right) \sim 0.1$ (typical of the low $m_{t^{\prime}}$ results given in Hou and Stewart, see Ref. [7]), we obtain about $67(51) b \bar{b} \gamma \gamma$ events. In order for these events to be sufficiently free of background, single $b$ tagging is probably necessary. If we assume that the efficiency for tagging of at least one $b$ (after appropriate kinematical cuts) is approximately $50 \%$ (as found in the most recent CDF top-quark analysis ${ }^{[20]}$ ) and that the overall cuts/acceptance/detection efficiency (after requiring minimum transverse momentum, say $p_{T}>15 \mathrm{GeV}$, and central rapidity for the photons and $b$ 's) is about $35 \%$, we see that a clean sample of 11(8) or so events should be present in the CDF data. Of course, $t^{\prime} \bar{t}^{\prime}$ production will also contribute to the $b \bar{b} \gamma \gamma X$ signal assuming $t^{\prime} \rightarrow b^{\prime} W^{*}$ is the dominant decay of the $t^{\prime}$. (For $m_{t^{\prime}}$ near $m_{b^{\prime}}$ the jets or leptons from the $W^{*}$ will be quite soft, and the signal rather similar to that for direct $b^{\prime} \bar{b}^{\prime}$ production). Events of the $2 \gamma+2 j$ type with at least one tagged $b$ quark are currently under investigation by the CDF collaboration, ${ }^{[33]}$ and we expect that CDF will shortly be able to severely restrict the allowed four-generation scenarios. Since event rates rise rapidly with decreasing $m_{b^{\prime}}$, it is hard to imagine that a $b^{\prime}$ with mass much below $80-90 \mathrm{GeV}$ would escape detection. Referring to Fig. 2, this means that the $m_{b^{\prime}}\left(m_{b^{\prime}}\right)<75-85 \mathrm{GeV}$ region for $\tan \beta=1.5$ and the entire $\tan \beta=2.2$ region are on the verge of elimination if no signal is reported. An integrated Tevatron luminosity of $L=150 \mathrm{pb}^{-1}$ would surely allow one to use the $b \bar{b} \gamma \gamma$ final state to eliminate all $m_{b^{\prime}} \lesssim m_{b}+m_{Z}$, extending up to $m_{b^{\prime}}$ values such that the $b^{\prime} \rightarrow b Z$ mode really becomes significant enough to suppress the $b^{\prime} \rightarrow b \gamma$ branching ratio. At this point, as described above, one looks for extra $Z$ 's, which also provide an excellent signal.

Of course, if a reasonable $K$ factor is included in the $b^{\prime} \bar{b}^{\prime}$ cross section $(K=1.3$ to 1.5$)$, the event rates for the $b \bar{b} \gamma \gamma$ and/or extra $Z$ events will increase above those given above. This leaves even less room for the four-family model to avoid detection.

The only possible escape from the $b^{\prime} \rightarrow b \gamma$ and $b^{\prime} \rightarrow b Z$ decay mode constraints would be if $b^{\prime} \rightarrow b h^{0}$ decay is kinematically allowed. If allowed, it will certainly dominate all other modes when $b^{\prime} \rightarrow b Z$ is kinematically forbidden. Even when the $b^{\prime} \rightarrow b Z$ channel is open, $b^{\prime} \rightarrow b h^{0}$ will at the very least be competitive and suppress the $b^{\prime} \rightarrow b Z$ branching ratio - indeed, the $b h^{0}$ channel could still be the dominant mode. ${ }^{[34]}$ However, as seen in Fig. 6 (after converting running masses to pole masses), this escape is not available for the $m_{t^{\prime}}>m_{b^{\prime}}$ portion of parameter space in the case of the dilaton boundary condition scenario, since there $m_{b^{\prime}}<m_{h^{0}}$. Only in the extreme lower right-hand corner where the running masses obey $m_{h^{0}} \simeq m_{b^{\prime}} \simeq 100 \mathrm{GeV}$ and $m_{t^{\prime}} \simeq 50 \mathrm{GeV}$, is the $b^{\prime} \rightarrow b h^{0}$ decay kinematically allowed, and this corner is almost certainly ruled out (as discussed in an earlier paragraph) since $m_{t^{\prime}}<m_{b^{\prime}}$. However, for scenarios with larger (but not too large) $m_{0}$, the $h^{0}$ can be light enough to make the $b h^{0}$ channel kinematically accessible.

In Fig. 11 we plotted as a function of $\xi_{A}$, for various fixed $\xi_{0}$ values, the 
minimum $m_{\widetilde{g}}\left(m_{\tilde{g}}\right)$ value that is allowed by the $\widetilde{\tau}_{1}^{\prime}$ constraints, taking $m_{b^{\prime}}\left(m_{b^{\prime}}\right)=$ $m_{t^{\prime}}\left(m_{t^{\prime}}\right)=100 \mathrm{GeV}$ (corresponding to pole masses of about $105 \mathrm{GeV}$ ). We can now use this minimum $m_{\tilde{g}}\left(m_{\tilde{g}}\right)$ value to fix all other model parameters, thereby determining the sparticle spectrum, in particular $m_{h^{0}}$. Since the $m_{H_{1,2}}^{2}$ contributions to the $m_{i}^{2}=m_{H_{i}}^{2}+\mu^{2}$ mass terms in the scalar potential will tend to be smallest when $m_{\tilde{g}}$ is small (and $\mu$ also tends to scale with $m_{\tilde{g}}$ ), these minimum $m_{\widetilde{g}}$ values give $m_{h^{0}}$ values that will be very close to the minimum allowed for the fourth-generation fermion masses chosen. To determine if $b^{\prime} \rightarrow h^{0} b$ is kinematically allowed, we compute $m_{b^{\prime}}($ pole $)-m_{h^{0}}-m_{b}$. Plots of $m_{h^{0}}$ and this mass difference also appeared in Fig. 11. From the $m_{b^{\prime}}($ pole $)-m_{h^{0}}-m_{b}$ curves, we see that for middle-of-the-road values of $\xi_{0}$ the $b^{\prime} \rightarrow b h^{0}$ decay can be kinematically allowed. When the $b^{\prime} \rightarrow b h^{0}$ decay channel is present, thereby suppressing the $b^{\prime} \rightarrow b Z$ channel branching ratio, a $b^{\prime}$ with pole mass somewhat above $105 \mathrm{GeV}$ could have escaped observation.

\section{$\underline{t^{\prime} \bar{t}^{\prime} \text { Production at the Tevatron }}$}

Let us now turn to a more thorough examination of $t^{\prime} \bar{t}^{\prime}$ events. For the $m_{t^{\prime}}>$ $100 \mathrm{GeV}$ mass region, there are two competing decay modes for the $t^{\prime}: t^{\prime} \rightarrow b^{\prime} W^{(*)}$ and $t^{\prime} \rightarrow b W$. The $b^{\prime} W$ decay will certainly dominate over the $b W$ mode when the former $W$ is real, since the $b W$ channel is suppressed by the intra-generational mixing factor $V_{t^{\prime} b}$. However, when $m_{t^{\prime}}-m_{b^{\prime}}<m_{W}$, the two-body $b W$ mode could be competitive with the three-body $b^{\prime} W^{*}$ decay. In Fig. 17 we plot the value of $V_{t^{\prime} b}$ for which $\Gamma\left(t^{\prime} \rightarrow b^{\prime} W^{*}\right)=\Gamma\left(t^{\prime} \rightarrow b W\right)$ as a function of $m_{t^{\prime}}$ for several $m_{b^{\prime}}$ values (we employ pole masses). For $V_{t^{\prime} b}$ larger than the values plotted, the $t^{\prime} \rightarrow b W$ mode is dominant. We see that for $b^{\prime}$ (pole) masses larger than the rough lower bound of $80 \mathrm{GeV}$, discussed above, it is not at all impossible that $V_{t^{\prime} b}$ could be large enough for $t^{\prime} \rightarrow b W$ to be the dominant mode.

For $m_{t^{\prime}} \lesssim 170 \mathrm{GeV}$ (as required by our Yukawa perturbativity bound), such dominance would imply a large excess of events relative to those already present by virtue of $t \bar{t}$ production and decay. As discussed in more detail below, the CDF and D0 experimental results are now more or less consistent with the expected $t \bar{t}$ rates for $m_{t} \sim 175-185 \mathrm{GeV}$. Taken at face value, these results then imply that $t^{\prime} \rightarrow b W$ cannot be the dominant $t^{\prime}$ decay mode.

In fact, we show below that it is only possible to obtain sufficiently few CDF and D0 events if the $t^{\prime}$ decay is to $b^{\prime}$ plus a highly virtual $W^{*}$. A typical case that survives is $m_{t^{\prime}} \sim 115 \mathrm{GeV}$ and $m_{b^{\prime}} \sim 80 \mathrm{GeV}$. From Fig. 17 we find that $V_{t^{\prime} b} \lesssim 0.02$ (a value roughly the same as $V_{c b}$ ) is required for $t^{\prime} \rightarrow b W$ to be adequately suppressed for these mass choices. Such values for $V_{t^{\prime} b}$ are certainly reasonable; in fact, one might expect considerably smaller values based on the fact 
that $V_{b c} \ll V_{u s}$, but we must also consider implications for $b^{\prime}$ decay. We noted earlier that if $b^{\prime} \rightarrow c W$ were the dominant $b^{\prime}$ decay mode, then $b^{\prime} \bar{b}^{\prime}$ events would have been detected. Consistency with experiment thus requires that the one-loop $b^{\prime} \rightarrow b X$ neutral current decays are dominant. This, in turn, requires (see Hou and Stewart, Ref. [7]) that $V_{t^{\prime} b} / V_{b^{\prime} c}, V_{t b^{\prime}} / V_{b^{\prime} c} \gtrsim 10^{2}-10^{3}$. Combining with the $V_{t^{\prime} b}$ restriction above, we find that $V_{b^{\prime} c} \lesssim 10^{-4}$ is required. As seen from Fig. 17, this limit would become stronger for larger values of $m_{b^{\prime}}$. Suppressions larger than those suggested by measured $V_{c b}$ and $V_{u b}$ values would be required for $m_{b^{\prime}}$ values much above $100 \mathrm{GeV}$ and thus become problematical. As discussed earlier, $m_{b^{\prime}} \gtrsim 100 \mathrm{GeV}$ is also the range for which $b^{\prime} \rightarrow h^{0} b$ decay dominance is probably required to avoid too many extra $Z$ events from $b^{\prime} \rightarrow Z b$ decays in $b^{\prime} \bar{b}^{\prime}$ events.

In our previous work, ${ }^{[1]}$ we performed a rough Monte-Carlo study of the number of $t^{\prime} \bar{t}^{\prime}$ production and decay events expected in the dilepton-plus-jets and leptonplus-jets channels, assuming that $t^{\prime} \rightarrow b^{\prime} W^{(*)}$ is the primary $t^{\prime}$ decay. At the time, the number of additional events (beyond the predicted number from $t \bar{t}$ production) in these two channels was not inconsistent with the then-available CDF data for any of the larger $m_{t^{\prime}}, m_{b^{\prime}}$ choices within the perturbatively allowed region. However, the luminosity accumulated by both CDF and D0 is now much larger $\left(L \sim 67 \mathrm{pb}^{-1}\right.$ vs. $\left.19 \mathrm{pb}^{-1}\right)$. Further, CDF now employs new $b$-tagging algorithms which roughly double their $b$-tagging efficiency. Consequently, the constraints on the model are now much stronger. Exactly how severe the constraints are depends on the precise predictions for event rates from normal $t \bar{t}$ production. The CDF collaboration states that their results are in good agreement with a top-quark Monte Carlo. As a cross check, we have also repeated the Monte Carlo study of our previous work, Ref. [1], after adjusting for the new luminosity and new $b$-tagging procedures. Despite the approximate nature of our implementation of the CDF cuts and $b$ tagging procedures, agreement is good. For $m_{t}=175 \mathrm{GeV}$, the uncut $t \bar{t}$ cross section is about $3.5 \mathrm{pb}$, and roughly 25 events pass the single-lepton plus $b$-tag plus $\geq 3$-jets criteria, while $\sim 3$ events pass the dilepton cuts. CDF observes 37 events in in the $W+\geq 3$ jet channel in which at least one $b$ is tagged, with an estimated background of 16, and 6 events in the di-lepton channel, with estimated background of 1.3. Thus, their number of signal events is 21 and 4.7 in these two respective channels, to be compared to 25 and 3. Clearly, there is little room for additional events passing their cuts from $t^{\prime} \bar{t}^{\prime}$ production.

The rates (after implementing the same cuts, etc.) in these two channels deriving from $t^{\prime} \bar{t}^{\prime}$ production, followed by FCNC decays of the type $b^{\prime} \rightarrow b+j e t(s)$ (in particular, no extra photons or leptons from $b^{\prime}$ decay are allowed) appear in Table 3. (In Table 3 we have included several $\left(m_{t^{\prime}}, m_{b^{\prime}}\right)$ pairs that are excluded by the boundaries of Fig. 2 just to indicate the effects of different mass choices on the rates.) Restrictions on the perturbatively allowed regions of Fig. 2 are now significant. For $\tan \beta=2.2$, for which only values of $m_{b^{\prime}}<85 \mathrm{GeV}$ (i.e.

* The number of background events, 16 , in the $W+\geq 3$-jets channel is estimated by scaling the tag background estimate of 22 events by the ratio $37 / 50$ of events/tags. 
Table 3: For $L=67 \mathrm{pb}^{-1}$, we tabulate the number of $t^{\prime} \bar{t}^{\prime}$ events passing our approximations to the CDF top-quark discovery cuts in the single lepton $+b$-tag and dilepton modes.

\begin{tabular}{c|cccc|cccc}
\hline \hline & \multicolumn{3}{|c|}{ Lepton $+b$-tag Mode } & \multicolumn{5}{|c}{ Dilepton Mode } \\
\cline { 2 - 8 } & \multicolumn{4}{|c|}{$m_{b^{\prime}}(\mathrm{GeV})$} & \multicolumn{5}{c}{$m_{b^{\prime}}(\mathrm{GeV})$} \\
$m_{t^{\prime}}(\mathrm{GeV})$ & 50 & 80 & 110 & 130 & 50 & 80 & 110 & 130 \\
\hline 160 & 20 & 16 & 3.6 & 1.5 & 2.4 & 1.8 & 0.2 & 0.01 \\
130 & 53 & 20 & 0.5 & - & 4.2 & 1.1 & 0.002 & - \\
100 & 58 & 3.6 & - & - & 2.8 & 0.01 & - & - \\
\hline \hline
\end{tabular}

$m_{b^{\prime}}\left(m_{b^{\prime}}\right) \lesssim 80 \mathrm{GeV}$ are perturbatively allowed, Table 3 shows $t^{\prime} \bar{t}^{\prime}$ production will yield sufficiently few CDF single-lepton $+b$-tag events only if $m_{t^{\prime}} \lesssim 105-115 \mathrm{GeV}$, effectively eliminating the upper $50 \%$ of the allowed $m_{t^{\prime}}\left(m_{t^{\prime}}\right)$ region. For $m_{t^{\prime}} \lesssim$ $105-115 \mathrm{GeV}$, Table 3 also shows that $m_{b^{\prime}}$ can't be too light; roughly $m_{b^{\prime}} \gtrsim 70 \mathrm{GeV}$ is required. The underlying reason for these restrictions is easily summarized. For $m_{t^{\prime}} \lesssim m_{t}$, the only way the $t^{\prime} \bar{t}^{\prime}$ events can evade being included in the CDF event sample is if $m_{t^{\prime}}-m_{b^{\prime}}$ is sufficiently small that the $W$ in $t^{\prime} \rightarrow W b^{\prime}$ is virtual and the jets and leptons from the two $W^{*}$ s are quite soft. For such cases, additional $t^{\prime} \bar{t}^{\prime}$ events could be included in the event sample if CDF and D0 could soften their cuts. However, backgrounds probably increase dramatically. If this is the case, then additional luminosity is the only way to improve sensitivity; for example, $L=150 \mathrm{pb}^{-1}$ would yield about $8 \mathrm{CDF}$ events (for current cuts and $b$-tagging efficiency) if $m_{t^{\prime}}=100$ and $m_{b^{\prime}}=80$. This would probably constitute a detectable signal.

\section{$\underline{\text { Combined Restrictions }}$}

Combining the $t^{\prime}$ restrictions with the existing and probable $b^{\prime}$ CDF data constraints, and accounting for the limited range allowed for $m_{b^{\prime}}$ by perturbativity (especially for $\tan \beta=2.2$ ) and the $m_{t^{\prime}}>m_{b^{\prime}}$ requirement, we are clearly forced into the running mass domain $110 \mathrm{GeV} \gtrsim m_{t^{\prime}}\left(m_{t^{\prime}}\right) \gtrsim m_{b^{\prime}}\left(m_{b^{\prime}}\right), 95 \mathrm{GeV} \gtrsim m_{b^{\prime}}\left(m_{b^{\prime}}\right) \gtrsim$ $75 \mathrm{GeV}$. For $\tan \beta=1.5$ this is the region that is as close to a fixed-point limit as the perturbativity constraints allow and is also the region for which the unification prediction for $\alpha_{s}\left(m_{Z}\right)$ is smallest and, thus, in best agreement with data. We have also seen that it is a region for which $V_{t^{\prime} b}$ and $V_{b^{\prime} c}$ can both take on values that are reasonable extraplolations from those already measured in the lighter quark sectors, while at the same time being consistent with dominance of $t^{\prime} \rightarrow b^{\prime} W^{*}$ over (probably excluded) $t^{\prime} \rightarrow b W$ decays in $t^{\prime} \bar{t}^{\prime}$ events and of $b^{\prime} \rightarrow X b$ neutral current decays over (probably excluded) $b^{\prime} \rightarrow c W$ decays in $b^{\prime} \bar{b}^{\prime}$ events, provided $m_{t^{\prime}}\left(m_{b^{\prime}}\right)$ lies in the upper (lower) portion of its allowed region. A tripling of the Tevatron's

$\dagger$ Note that the $t^{\prime} \rightarrow \widetilde{b}_{1}^{\prime} \widetilde{\chi}_{1}^{+}$decay, that could provide an escape from these restrictions, is not kinematically allowed for any of the mass spectra scenarios of Figs. 13-15. 
integrated luminosity to $L \sim 150 \mathrm{pb}^{-1}$ would almost certainly either close the $m_{b^{\prime}}$ window above, or lead to $b^{\prime}$ discovery.

The Hidden-Top Scenario

To escape from the experimental constraints on $t^{\prime} t^{\prime}$ production and decay discussed above would require that the 'top-quark' events not come from thirdgeneration $t \bar{t}$ production. As described earlier, in one such scenario $m_{t} \sim m_{W}$ and $t \rightarrow \widetilde{t}_{1} \widetilde{\chi}_{1}^{0}$ decays are dominant. Typical (pole) masses required for this scenario to be viable are those discussed in Ref. [14]: $m_{t} \sim m_{W}, m_{t^{\prime}} \sim 170 \mathrm{GeV}$, $m_{b^{\prime}} \sim 110 \mathrm{GeV}, m_{\widetilde{\chi}_{1}^{0}} \lesssim 25 \mathrm{GeV}$, and $m_{\tilde{t}} \lesssim 60 \mathrm{GeV}$. We have explicitly analyzed this scenario using our full SUSY GUT framework. We find that for $m_{\tau^{\prime}}=50 \mathrm{GeV}$ (the choice of Ref. [14]) and $m_{\nu^{\prime}} \lesssim 78 \mathrm{GeV}$, the above $m_{t}, m_{t^{\prime}}$ and $m_{b^{\prime}}$ masses are consistent with gauge coupling unification and with perturbative evolution for the Yukawas up to the scale $M_{U}$. (Our criteria do not quite allow consistency with Yukawa perturbativity for the $m_{\nu^{\prime}}=80 \mathrm{GeV}$ choice of Ref. [14].)

However, we find that it is not possible to obtain $m_{\widetilde{\chi}_{1}^{0}}+m_{\widetilde{t}_{1}}<m_{t}$ (where $\widetilde{t}_{1}$ is the lighter stop mass eigenstate) in the context of SUSY GUT scenarios with universal $m_{0}$ and $A$ parameters at $M_{U}$. Since both $m_{\widetilde{\chi}_{1}^{0}}$ and $m_{\widetilde{t}_{1}}$ scale with $m_{\tilde{g}}$, the minimum value for the mass sum will occur at the smallest possible value of $m_{\tilde{g}}$. As described earlier, the minimum $m_{\tilde{g}}$ (or equivalently $m_{1 / 2}$ ) value is set by the $\widetilde{\tau}_{1}^{\prime}$ constraint. We find that the $\widetilde{\tau}_{1}^{\prime}$ constraint (for the mass scenario just outlined) implies an absolute lower bound on the $M_{U}$-scale parameter $m_{1 / 2}$ that is similar in nature to that illustrated in Fig. 10, except that lower values of $m_{1 / 2}$ (close to $100 \mathrm{GeV}$ ) are allowed at high $\xi_{0}$. This translates into $m_{\tilde{g}} \gtrsim 150 \mathrm{GeV}$. Analogously to Fig. 11, much higher values are required when $\xi_{0} \equiv m_{0} / m_{1 / 2} \lesssim 1$. Also as in Fig. 11, the minimum value of $m_{1 / 2}$ is achieved for a range of $\xi_{A} \equiv A / m_{1 / 2}$ values centered about 0 which is broad for large $\xi_{0}$, but increasingly narrow for $\xi_{0} \lesssim 1$. However, even at large $\xi_{0}$, arbitrariy large values of $\left|\xi_{A}\right|$ do not yield consistent solutions for any $m_{1 / 2}$, as illustrated by the termination of the low- $\xi_{0}$ curves in Fig. 11. Too large a value for $\left|\xi_{A}\right|$ (typically $\left|\xi_{A}\right| / \xi_{0} \gtrsim 4-5$ at large $\xi_{0}$ ) results in either $m_{A^{0}}^{2}<0$ at the EWSB minimum, and/or $m^{2}<0$ for one of the colored and/or charged 4th-family sparticles.

We describe two typical cases: $\xi_{0} \sim 1$ and $\xi_{0} \sim 5$. For $\xi_{0} \sim 1, m_{\tilde{g}} \gtrsim 400 \mathrm{GeV}$ is required, with $m_{\widetilde{\chi}_{1}^{0}} \sim 0.125-0.13 m_{\widetilde{g}}$ implying $m_{\widetilde{\chi}_{1}^{0}} \gtrsim 50 \mathrm{GeV}$. For $\xi_{0} \gtrsim 5$, we find $m_{\widetilde{g}} \gtrsim 150 \mathrm{GeV}$ and $m_{\widetilde{\chi}_{1}^{0}} \sim 0.14 m_{\widetilde{g}}$, implying $m_{\widetilde{\chi}_{1}^{0}} \gtrsim 21 \mathrm{GeV}$. ( Both of these minimum values only apply for small $\xi_{A} / \xi_{0}$. As $\xi_{A} / \xi_{0}$ increases, the minimum allowed value of $m_{\widetilde{g}}$ increases, but very slowly in the case of $\xi_{0}=5$.) Although the latter $m_{\widetilde{\chi}_{1}^{0}}$ value is in an acceptable range for the scenario, $m_{\widetilde{t}_{1}}$ is always quite large for $\xi_{A}$ values that do not imply $m_{A^{0}}^{2}<0$ and/or color/U(1) breaking. This is because $m_{\widetilde{t}_{1}}$ is always well above $m_{t}=80 \mathrm{GeV}$ at small $\xi_{A}$ (typically by three 
hundred $\mathrm{GeV}$ or more for $\xi_{0}=5$ ). Thus, even though $\widetilde{t}_{L}-\widetilde{t}_{R}$ mixing increases so that $m_{\widetilde{t}_{1}}$ decreases as one moves to higher $\left|\xi_{A}\right|$ in the $\xi_{A}<0$ direction, $m_{\widetilde{t}_{1}}$ only declines by some $50 \mathrm{GeV}$ before $\left|\xi_{A}\right|$ is large enough that $m_{A^{0}}^{2}<0$ and/or color/U(1) breaking arises.

For allowed $\xi_{A}$ values, what prevents a sufficiently small mass for the $\widetilde{t}_{1}$ is the fact that the $\widetilde{\tau}_{1}^{\prime}$ cannot be too light. The simplest way to avoid this lock-step arrangement is to break the universality assumption for the $m_{0}$ parameters at $M_{U}$, allowing much smaller $m_{0}$ in the $\widetilde{t}$ sector than in the $\widetilde{\tau}^{\prime}$ sector of the theory. We have not explored this option.

\section{7b. Four-Family Precision EW tests ${ }^{\star}$}

In addition to direct collider searches for the presence of a possible fourth family, indirect effects arising from virtual quantum effects can be probed through precision measurements of many observables and can lead to indirect limits or bounds on the parameters of the theory. For example, the well-known $\rho$-parameter limits the magnitude of iso-spin breaking, and thus bounds the $m_{t^{\prime}}^{2}-m_{b^{\prime}}^{2}$ and $m_{\tau^{\prime}}^{2}-m_{\nu^{\prime}}^{2}$ splittings of a possible fourth family. On the other hand, although the LEP measurement of $Z \rightarrow b \bar{b}$ is a particularly sensitive probe to any new physics involving possible coupling to the top quark, the vertex contribution to $Z \rightarrow b \bar{b}$ arising from a fourth family is expected to be small due to CKM mixing suppression. We therefore neglect all vertex contributions in our analysis, focusing instead on vacuum polarization effects.

Overall, it desirable to perform a global fit to the present experimental data using several different observables. The most comprehensive approach would be to perform the complete, one-loop calculation for each observable in question, and do a best fit of the new physical parameters of the model, (i.e. $m_{t^{\prime}}, m_{b^{\prime}}, m_{\tau^{\prime}}$, $\left.m_{\nu^{\prime}}, \tan \beta, \mu, \ldots\right)$. This rather ambitious approach has only recently been attempted for the MSSM, and will not be attempted here. A more modest approach inevitably involves making certain assumptions and approximations. i) As previously explained, a fourth-family added to the MSSM contributes dominantly via 'oblique', or vacuum polarization effects. ii) We assume that the contributions from the sparticles are small enough to be neglected. This is likely to be a good approximation for $N_{g}=4$ models. Indeed, we have seen that for $N_{g}=4$ the supergravity boundary conditions and evolution equations imply a fairly large lower bound on $m_{\widetilde{g}}$ (especially for the dilaton and no-scale boundary condition choices) which, in turn, implies that most of the sparticle masses are large. According to the decoupling theorem, loops involving massive SUSY particles contribute negligibly to vacuum polarization amplitudes. The particles most likely to violate this approximation are the sometimes-light $\widetilde{\tau}_{1}^{\prime}$ and $\widetilde{\nu}_{1}^{\prime}$, and even these are heavy for

\footnotetext{
$\star$ In this sub-section we will exclusively employ running masses.
} 
larger $m_{\tilde{g}}$ values, independent of model. Finally, iii) we assume that the $h^{0}$ of the Higgs sector is SM-like, implying that the SUSY Higgs sector is equivalent to a SM Higgs sector with a light Higgs boson. Given the four-family constraint $\tan \beta \lesssim 3$, this is true for $m_{A^{0}} \gtrsim 200 \mathrm{GeV}$, as is essentially always the case for the models considered.

In the following analysis, we perform a global fit to the latest LEP+SLD data ${ }^{[35]}$ and we employ an extension of the Peskin/Takeuchi S,T,U formalism ${ }^{[36]}$ in order to find the $90 \%, 95 \%$ allowed regions of parameter space. In this approach, for a given 'reference' $\mathrm{SM}$ (i.e. with chosen $m_{t}, m_{H}$ values), $S, T, U \equiv 0$, and the (non-zero) best-fit values to experimental data are a measure of the oblique, or vacuum polarization, contributions that should be explained by 'new physics'. For our reference SM we adopt $N_{g}=3$ with $m_{t}\left(m_{t}\right)=165 \mathrm{GeV}$ and Higgs mass $m_{H}=100 \mathrm{GeV}$ (typical of the $m_{h^{0}}$ values found in our computations). We then treat the contributions from $t^{\prime}, b^{\prime}, \nu^{\prime}, \tau^{\prime}$ as new physics.

At this point, there are several parameter choices and assumptions that affect our results. i) What values of $\alpha_{e m}\left(m_{Z}\right), \alpha_{s}\left(m_{Z}\right)$ are chosen for the reference SM? As was recently shown, new estimates of $\alpha_{e m}\left(m_{Z}\right)$ can shift the best-fit for the experimental value of the $S$-parameter relative to $S=0$ for the reference SM by $+10 \%$. Similarly, the choice of $\alpha_{s}\left(m_{Z}\right)$ is quite important. Secondly, ii) one should be careful when using the $S, T, U$ formalism in the presence of new light physics, since in this case it has recently been shown that the standard $S, T, U$ formalism is simply not an adequate parameterization. ${ }^{[37,38]}$ In the original work of Peskin and Takeuchi, the $S, T, U$ parameters were explicitly defined using the linear approximation in an expansion in $q^{2} / M_{Z}^{2}{ }^{[36]}$ Although perfectly valid when considering new physics with scales much higher than $m_{Z}$, such as technicolor, this expansion is not accurate for new physics near or below $m_{Z}$. It is possible to define a set of parameters which do not rely on a $q^{2} / M_{Z}^{2}$ expansion. In a complete comparison to all available experimental observables, this results in a proliferation of new parameters that must be simultaneously fit. However, if one restricts the 'global' fit to Z-pole plus $m_{W}^{2} / m_{Z}^{2}$ measurements, then one can show that a redefinition of $S \rightarrow S^{\prime}, T \rightarrow T^{\prime}, U \rightarrow U^{\prime}$ is possible, where the primed variables can be computed in the presence of additional light physics without approximation. Further all the observables in question are linear functions of $S^{\prime}, T^{\prime}, U^{\prime}$. Thus, the procedure is to restrict the fit to measurables which are defined at the Z-peak (along with the $m_{W} / m_{Z}$ measurement), employ the standard three-parameter $S, T, U$ global fit to the experimental data (relative to a reference SM with $S=T=U=0$ by definition), and finally re-interpret the best fit $S, T, U$ values as $S^{\prime}, T^{\prime}, U^{\prime}$.

In order to compare to predictions of a four-generation model, we calculate the exact (no expansion is performed) contributions to $S^{\prime}, T^{\prime}, U^{\prime}$ from loops involving

$\dagger$ In a quadratic expansion, in general seven independent parameters arise ${ }^{[37,38]}$, and in a fully exact treatment, eleven parameters must be specified ${ }^{[39]}$ 
fourth-generation particles for a given set of $m_{t}, m_{t^{\prime}}, m_{b^{\prime}}, m_{\nu^{\prime}}, m_{\tau^{\prime}}$ values; denote these contributions as $S_{4}^{\prime}, T_{4}^{\prime}, U_{4}^{\prime}$. Since the observables $\mathcal{O}$ are linear functions of $S^{\prime}, T^{\prime}, U^{\prime}$, we can then compute $\mathcal{O}_{i}\left(N_{g}=4\right) \equiv \mathcal{O}_{i}\left(S_{4}^{\prime}, T_{4}^{\prime}, U_{4}^{\prime}\right)$. The predicted values of $\mathcal{O}_{i}\left(N_{g}=4\right)$ are then compared to the best fit experimental values as obtained using ZFITTER (relative to the reference SM assumed) and an overall $\Delta \chi^{2}$ computed. This computation is performed for each point of interest in the fourth-generation parameter space. As noted earlier, we use a reference SM with $m_{t}\left(m_{t}\right)=165 \mathrm{GeV}$ and $m_{H}=100 \mathrm{GeV}$ and take $\alpha_{\text {em }}^{-1}\left(m_{Z}\right)=129.08^{[17]}$. We employ the latest values for the following measurements: $m_{W} / m_{Z}, \Gamma_{Z}, \sigma_{h}^{0}, R_{l}, R_{b}, R_{c}, A_{F B}^{0, l}$, $A_{F B}^{0, b}, A_{F B}^{0, c}, A_{\tau}, A_{e}$ from LEP and $A_{L R}^{0}$ from SLD. ${ }^{\left[{ }^{35]}\right.}$ Finally, because of sensitivity of the reference SM prediction from ZFITTER for these observables to $\alpha_{s}$, we give results for the two values, $\alpha_{3}\left(m_{Z}\right)=(0.12,0.13)$.

The solid and dashed lines in Fig. 18 summarize the final results of this global fit. There, we have adopted $m_{\tau^{\prime}}=m_{\nu^{\prime}}=50 \mathrm{GeV}, \tan \beta=1.5$ and have allowed $m_{t^{\prime}}\left(m_{t^{\prime}}\right)$ and $m_{b^{\prime}}\left(m_{b^{\prime}}\right)$ to vary. The region in the $m_{t^{\prime}}, m_{b^{\prime}}$ parameter space above the lower (upper) solid line is excluded at 90\%(95\%) C.L. if the reference SM has $\alpha_{3}\left(m_{Z}\right)=0.13$. The two dashed lines give the same C.L. boundaries in the case $\alpha_{3}\left(m_{Z}\right)=0.12$; in this latter case, Fig. 18 shows that there is a region at small $m_{t^{\prime}}$ that is also excluded. As one might have naively anticipated, the region of large $m_{t^{\prime}}$ compared to $m_{b^{\prime}}$ has a poor C.L.; however, we also see that if both $m_{b^{\prime}}$ and $m_{t^{\prime}}$ are small, then predictions of the fourth-generation model for $S^{\prime}, T^{\prime}, U^{\prime}$ can again deviate significantly from the experimentally preferred values, depending upon $\alpha_{s}\left(m_{Z}\right)$. The region of modest $m_{t^{\prime}}\left(m_{t^{\prime}}\right) \sim m_{b^{\prime}}\left(m_{b^{\prime}}\right) \sim 100 \mathrm{GeV}$, preferred on the basis of current and anticipated Tevatron results, lies well within even the $90 \%$ C.L. limits.

\section{7c. Influence on jet and di-jet spectra of a slowly running four-family $\alpha_{s}$}

One other indirect effect of the presence of a fourth family, along with its full complement of supersymmetric partners, all at relatively low energy scales, has recurred throughout our discussion: $\alpha_{s}(Q)$ decreases much more slowly with increasing $Q$ than in a standard three-family model, especially if the comparison is with the case where the masses of the superpartners of the three-family model are taken to be large. Thus, it is important to not forget that $\alpha_{s}(Q)$ is, in fact, directly measurable through the single-jet and di-jet spectra of light quark jets. Of course, the exact scale $Q$ at which $\alpha_{s}$ is evaluated will be an important issue. In the $\overline{M S}$ scheme, higher order calculations suggest that an appropriate $Q$ value for single-jet inclusive spectra and di-jet mass spectra is the subprocess center-of-mass energy, roughly given by $2 E_{T}$ and $m_{2 j}$, respectively. How large can the deviations be? To illustrate, we consider as a function of $Q$ the fractional enhancement ratio

$$
R_{\alpha_{s}} \equiv \frac{\left[\alpha_{s}\left(N_{g}=4\right)\right]^{2}-\left[\alpha_{s}^{S M}\left(N_{g}=3\right)\right]^{2}}{\left[\alpha_{s}^{S M}\left(N_{g}=3\right)\right]^{2}}
$$

where $\alpha_{s}^{S M}$ is the $\alpha_{s}$ predicted in the three-family case with no superpartner effects, (i.e. with all superparticle masses taken to be large); results for $\alpha_{s}\left(N_{g}=4\right)$ will 
be illustrated for $m_{b^{\prime}}($ pole $)=m_{t^{\prime}}($ pole $)=105 \mathrm{GeV}$ (our preferred mass range) and $M_{S U S Y}^{Q C D}$ values of $200 \mathrm{GeV}$ and $400 \mathrm{GeV}$. Such values are quite typical of the overall mass scales for the strongly-interacting sparticles (that contribute to $\alpha_{s}$ evolution) in the model scenarios we have studied. We employ $\alpha_{s}^{2}$ in Eq. (20) because this is what appears in the QCD subprocess cross sections.

The results appear in Fig. 19. We see that for $M_{S U S Y}^{Q C D}=200 \mathrm{GeV}$, percentage deviations are, for example, about $25 \%$ at $Q=1 \mathrm{TeV}$. This would correspond to subprocess energies of $1 \mathrm{TeV}$ for the standard $\overline{M S}$ scale choice mentioned above. The corresponding value for the (probably more reasonable) choice of $M_{S U S Y}^{Q C D}=$ $400 \mathrm{GeV}$ is $12 \%$. If there are only three families with superpartners at a light mass scale, the values of $R_{\alpha_{s}}$, Eq. (20)( with $\alpha_{s}\left(N_{g}=3\right.$ ) in place of $\alpha_{s}\left(N_{g}=4\right)$ ) are very much smaller in all cases. Thus, it is not entirely impossible that preliminary observations of this type of deviation by CDF and D0 ${ }^{[40]}$ could be a hint that four generations with relatively light superpartners are present.

\section{Conclusions}

We have shown that a four-generation MSSM model is an attractive extension of the usual three-generation MSSM in that gauge unification and automatic electroweak symmetry breaking via RGE renormalization both occur naturally for typical soft-SUSY-parameter boundary conditions at $M_{U}$. A significant, but restricted range of $t^{\prime}$ and $b^{\prime}$ masses is allowed (see below) even after requiring that all Yukawa couplings remain in the perturbative domain throughout $m_{Z}$ to $M_{U}$ evolution; however, Yukawa unification is generally not possible. The domain of allowed $t^{\prime}$ and $b^{\prime}$ masses is impacted by constraints coming from the SUSY sparticle sector, the most important of which is the requirement that the lighter $\widetilde{\tau}^{\prime}$ eigenstate $\left(\widetilde{\tau}_{1}^{\prime}\right)$ be more massive than the LEP-I limit of $45 \mathrm{GeV}$ and also heavier than the $\widetilde{\chi}_{1}^{0}$ LSP. This constraint can rule out a range of lighter $b^{\prime}$ masses; the smaller the soft-SUSY-breaking $m_{0}$ and $m_{1 / 2}$ parameters, the larger the range. For given $t^{\prime}$ and $b^{\prime}$ masses the $\widetilde{\tau}_{1}^{\prime}$ constraint places significant restrictions on $m_{0}$ and $m_{1 / 2}$, and thence on other sparticle masses. Generally, small $m_{0}$ values are disfavored unless $m_{\widetilde{g}}$ is large, and there is a significant lower bound on $m_{\widetilde{g}}$ such that no $m_{0}, A$ choices yield a satisfactory $\widetilde{\tau}_{1}^{\prime}$ mass. There is no analogous bound in the three-generation case. Thus, the sparticle mass scale must be larger in four-vs. three-generation MSSM models.

$\ddagger$ Note that $M_{S U S Y}^{Q C D}$ is not generally the same as the effective $M_{S U S Y}$ appropriate in discussing gauge coupling unification, which is sensitive to the entire particle content of the theory and not just the strongly interacting particles. 
Significant differences are also found for the relations between different sparticle masses in comparing $N_{g}=4$ results to those for $N_{g}=3$. The most dramatic difference arises directly from the roughly factor of two larger value of the unified gauge coupling $\alpha\left(M_{U}\right)$ in the $N_{g}=4$ case. This leads to ratios such as $m_{\widetilde{\ell}_{R}} / m_{\widetilde{\chi}_{1}^{0}}$ and $m_{\widetilde{\ell}_{L}} / m_{\widetilde{\chi}_{1}^{0}}$ being approximately a factor of two larger for $N_{g}=4$ compared to $N_{g}=3$ for a given choice of $m_{0} / m_{1 / 2}$. Indirect tests/verifications of the presence of a fourth generation by observing only a light gaugino and sparticles belonging to the first two families are thus possible.

Direct experimental constraints on the perturbative four-generation MSSM model are becoming very strong, and near-term experiments could easily eliminate the model altogether. The $\nu^{\prime}$ and $\tau^{\prime}$ have masses that are strongly bounded from above for all $m_{t^{\prime}}, m_{b^{\prime}}>45 \mathrm{GeV}$ (the current LEP-I bound) and will be readily seen at LEP-176. For $\nu^{\prime}$ and $\tau^{\prime}$ masses just above the current LEP lower bounds (we take $50 \mathrm{GeV}$ for these masses) perturbativity constraints force $m_{t^{\prime}}($ pole $) \lesssim 160 \mathrm{GeV}$ and $m_{b^{\prime}}($ pole $) \lesssim 120 \mathrm{GeV}$. We also can be quite certain that the $t^{\prime}$ is heavier than the $b^{\prime}$ since otherwise the $t^{\prime}$ decays to $b W$ (or $b W^{*}$ ), and $t^{\prime} t^{\prime}$ final states would have led to top-quark-like events at a rate inconsistent with CDF and D0 results. For $m_{t^{\prime}}$ (pole) $>m_{b^{\prime}}\left(\right.$ pole), the $b^{\prime}$ can (and must, if it is to have evaded detection to date) decay via flavor changing neutral current processes to $b+X$ $\left(X=\gamma, g, q \bar{q}, Z^{(*)}, h^{0}, \ldots\right)$. For expected branching ratios, non-observation of $b^{\prime} \bar{b}^{\prime}$ production events at the Tevatron in which both $b^{\prime}$ 's decay to $b+\gamma$ probably excludes $m_{b^{\prime}}($ pole $) \lesssim 80-85 \mathrm{GeV}$. The range $m_{b^{\prime}}($ pole $)>m_{Z}+m_{b}($ pole $)+\sim 5 \mathrm{GeV}$ (for which $b^{\prime} \rightarrow b Z$ decays have significant branching ratio) is probably excluded by the non-observation of the extra $Z$ 's expected from $b^{\prime} \bar{b}^{\prime}$ production events, except for soft-SUSY-breaking parameter scenarios such that $m_{h^{0}}$ is small enough for $b^{\prime} \rightarrow b h^{0}$ decays to dominate over $b^{\prime} \rightarrow b Z$ - the scenarios for which the $h^{0}$ channel can be open have a large soft-SUSY-breaking scalar mass, $m_{0}$. The top quark searches of CDF and D0 would have detected production of $t^{\prime} t^{\prime}$ with $t^{\prime} \rightarrow b^{\prime} W$ (or $W^{*}$ ) and $b^{\prime}$ decaying hadronically, unless $m_{t^{\prime}}\left(\right.$ pole) is quite close to $m_{b^{\prime}}$ (pole) (implying soft $W^{*}$ decay products that evade their hard cuts). We roughly estimate that $m_{t^{\prime}}$ (pole) must be $\lesssim 115-120 \mathrm{GeV}$ on this basis. All of this leads to a highly preferred mass range for the $b^{\prime}$ and $t^{\prime}$ :

$$
120 \mathrm{GeV} \gtrsim m_{t^{\prime}}(\text { pole }) \gtrsim m_{b^{\prime}}(\text { pole }) \quad \text { with } \quad 100 \mathrm{GeV} \gtrsim m_{b^{\prime}}(\text { pole }) \gtrsim 80 \mathrm{GeV}
$$

We estimate that, for an integrated luminosity of $L=150 \mathrm{pb}^{-1}$, it is well within the capability of the Tevatron to either exclude all reasonable allowed models or detect a signal.

Of course, some of the above restrictions follow from the assumption that the 'top-quark' events at CDF and D0 arise from the third-generation top. We have noted that the alternative case (emphasized in Ref. [14]) where these are $t^{\prime} \bar{t}^{\prime}$ events in which $t^{\prime} \rightarrow b W$, while the normal $t$ has $m_{t} \sim m_{W}$ and decays via the difficultto-detect $t \rightarrow \widetilde{t}_{1} \widetilde{\chi}_{1}^{0}$ channel is not possible in the SUSY GUT context assuming 
universal $m_{0}$ and $A$ parameters. As one adjusts parameters so as to minimize $m_{\widetilde{t}_{1}}+m_{\widetilde{\chi}_{1}^{0}}$, the $\widetilde{\tau}_{1}^{\prime}$ always becomes too light, its mass falling below the LEP-I bound or below the LSP mass, or the theory becomes inconsistent by virtue of the CP-odd Higgs or some colored and/or charged 4th-family sparticle being required to have $m^{2}<0$, long before the lighter $\tilde{t}_{1}$ reaches masses below $m_{W}$.

Certainly, if there is a fourth generation with Yukawa couplings that remain perturbative up to $M_{U}$, experimentalists will discover a plethora of new signals at LEP-II and with increased luminosity at the Tevatron. If in the end no signal is found, requiring (roughly) $m_{\tau^{\prime}}, m_{\nu^{\prime}} \gtrsim 95 \mathrm{GeV}$ and $m_{t^{\prime}}, m_{b^{\prime}} \gtrsim 200 \mathrm{GeV}$, determined four-generation model builders must become resigned to having one or more of the Yukawa coupling constants becoming non-perturbative before evolution up to $M_{U}$ is complete. While there is no known fundamental reason to disallow this, such a scenario is distinctly less predictive, and therefore less attractive, than full perturbative evolution up to the unification scale for all parameters.

\section{Acknowledgments}

This work has been supported in part by Department of Energy grants \#DEFG03-91ER40674, \#DE-FG02-85ER40214, and by the Davis Institute for High Energy Physics.

\section{APPENDIX}

In this Appendix we collect all of the renormalization group equations for the four-generation extension of the MSSM that are used in our calculations. Among the many references available, we primarily employed Bjorkman and Jones, Ref. [15], Martin and Vaughn, ${ }^{[41]}$ and Cvetic and Preitschopf ${ }^{[6]}$ for the various betafunctions required.

Assuming no KM mixing and universal scalar masses at $M_{U}$, and defining $t=$ $\left(\frac{1}{2 \pi}\right) \log [Q(\mathrm{GeV})], y_{i}=\lambda_{i}^{2} /(4 \pi)$, and $\alpha_{i}=g_{i}^{2} /(4 \pi)$, we summarize the RGE's below. We denote the anomalous-dimension contributions to $\frac{d y_{i}}{d t}$ by $4 \pi \gamma_{i}^{(1)}$ at one loop and $(4 \pi)^{2} \gamma_{i}^{(2)}$ at two loops for the $i^{\text {th }}$ (unmixed) fermion.

For the Yukawa couplings we write

$$
\frac{d y_{i}}{d t}=y_{i}\left(4 \pi \gamma_{i}^{(1)}+(4 \pi)^{2} \gamma_{i}^{(2)}\right)
$$

where $i=t, b, \tau, t^{\prime}, b^{\prime}, \nu^{\prime}, \tau^{\prime}$. The expressions for $4 \pi \gamma_{i}^{(1)}$ and $(4 \pi)^{2} \gamma_{i}^{(2)}$ are as follows: 


$$
\begin{aligned}
4 \pi \gamma_{t}^{(1)} & =-\frac{13}{15} \alpha_{1}-3 \alpha_{2}-\frac{16}{3} \alpha_{3}+6 y_{t}+3 y_{t^{\prime}}+y_{b}+y_{\nu^{\prime}} \\
(4 \pi)^{2} \gamma_{t}^{(2)} & =\frac{3523}{450} \alpha_{1}^{2}+\frac{27}{2} \alpha_{2}^{2}+\frac{80}{9} \alpha_{3}^{2}+\alpha_{1} \alpha_{2}+\frac{136}{45} \alpha_{1} \alpha_{3} \\
& +8 \alpha_{2} \alpha_{3}+16 y_{t} \alpha_{3}+16 y_{t^{\prime}} \alpha_{3}+\frac{6}{5} y_{t} \alpha_{1}+\frac{4}{5} y_{t^{\prime}} \alpha_{1} \\
& +\frac{2}{5} y_{b} \alpha_{1}+6 y_{t} \alpha_{2}-22 y_{t}^{2}-9 y_{t^{\prime}}^{2}-3 y_{\nu^{\prime}}^{2}-5 y_{b} y_{t} \\
& -3 y_{b^{\prime}} y_{t^{\prime}}-y_{\tau^{\prime}} y_{\nu^{\prime}}-5 y_{b}^{2}-9 y_{t} y_{t^{\prime}}-3 y_{b} y_{b^{\prime}}-y_{b} y_{\tau} \\
& -y_{b} y_{\tau^{\prime}}-3 y_{t} y_{\nu^{\prime}} ;
\end{aligned}
$$

$$
\begin{aligned}
4 \pi \gamma_{b}^{(1)} & =-\frac{7}{15} \alpha_{1}-3 \alpha_{2}-\frac{16}{3} \alpha_{3}+6 y_{b}+3 y_{b^{\prime}}+y_{t}+y_{\tau}+y_{\tau^{\prime}} \\
(4 \pi)^{2} \gamma_{b}^{(2)} & =\frac{371}{90} \alpha_{1}^{2}+\frac{27}{2} \alpha_{2}^{2}+\frac{80}{9} \alpha_{3}^{2}+\alpha_{1} \alpha_{2}+\frac{8}{9} \alpha_{1} \alpha_{3} \\
& +8 \alpha_{2} \alpha_{3}+16 y_{b} \alpha_{3}+16 y_{b^{\prime}} \alpha_{3}+\frac{2}{5} y_{b} \alpha_{1}+\frac{4}{5} y_{t} \alpha_{1} \\
& -\frac{2}{5} y_{b^{\prime}} \alpha_{1}+\frac{6}{5} y_{\tau} \alpha_{1}+\frac{6}{5} y_{\tau^{\prime}} \alpha_{1}+6 y_{b} \alpha_{2} \\
& -22 y_{b}^{2}-9 y_{b^{\prime}}^{2}-3 y_{\tau}^{2}-3 y_{\tau^{\prime}}^{2}-5 y_{b} y_{t}-3 y_{b^{\prime}} y_{t^{\prime}}-y_{\tau^{\prime}} y_{\nu^{\prime}} \\
& -5 y_{t}^{2}-9 y_{b} y_{b^{\prime}}-3 y_{t} y_{t^{\prime}}-3 y_{b} y_{\tau}-3 y_{b} y_{\tau^{\prime}}-y_{t} y_{\nu^{\prime}} ;
\end{aligned}
$$

$$
\begin{aligned}
4 \pi \gamma_{\tau}^{(1)} & =-\frac{9}{5} \alpha_{1}-3 \alpha_{2}+3 y_{b}+3 y_{b^{\prime}}+4 y_{\tau}+y_{\tau^{\prime}} \\
(4 \pi)^{2} \gamma_{\tau}^{(2)} & =\frac{171}{10} \alpha_{1}^{2}+\frac{27}{2} \alpha_{2}^{2}+\frac{9}{5} \alpha_{1} \alpha_{2}+16 y_{b} \alpha_{3}+16 y_{b^{\prime}} \alpha_{3} \\
& -\frac{2}{5} y_{b} \alpha_{1}-\frac{2}{5} y_{b^{\prime}} \alpha_{1}+\frac{6}{5} y_{\tau} \alpha_{1}+\frac{6}{5} y_{\tau^{\prime}} \alpha_{1}+6 y_{\tau} \alpha_{2} \\
& -9 y_{b}^{2}-9 y_{b^{\prime}}^{2}-10 y_{\tau}^{2}-3 y_{\tau^{\prime}}^{2}-3 y_{b} y_{t}-3 y_{b^{\prime}} y_{t^{\prime}}-y_{\tau^{\prime}} y_{\nu^{\prime}} \\
& -3 y_{\tau} y_{\tau^{\prime}}-9 y_{\tau} y_{b}-9 y_{\tau} y_{b^{\prime}}
\end{aligned}
$$




$$
\begin{aligned}
4 \pi \gamma_{t^{\prime}}^{(1)} & =-\frac{13}{15} \alpha_{1}-3 \alpha_{2}-\frac{16}{3} \alpha_{3}+6 y_{t^{\prime}}+3 y_{t}+y_{b^{\prime}}+y_{\nu^{\prime}} \\
(4 \pi)^{2} \gamma_{t^{\prime}}^{(2)} & =\frac{3523}{450} \alpha_{1}^{2}+\frac{27}{2} \alpha_{2}^{2}+\frac{80}{9} \alpha_{3}^{2}+\alpha_{1} \alpha_{2}+\frac{136}{45} \alpha_{1} \alpha_{3} \\
& +8 \alpha_{2} \alpha_{3}+16 y_{t^{\prime}} \alpha_{3}+16 y_{t} \alpha_{3}+\frac{6}{5} y_{t^{\prime}} \alpha_{1}+\frac{4}{5} y_{t} \alpha_{1} \\
& +\frac{2}{5} y_{b^{\prime}} \alpha_{1}+6 y_{t^{\prime}} \alpha_{2}-22 y_{t^{\prime}}^{2}-9 y_{t}^{2}-3 y_{\nu^{\prime}}^{2}-5 y_{b^{\prime}} y_{t^{\prime}} \\
& -3 y_{b} y_{t}-y_{\tau^{\prime}} y_{\nu^{\prime}}-5 y_{b^{\prime}}^{2}-9 y_{t^{\prime}} y_{t}-3 y_{b^{\prime}} y_{b}-y_{b^{\prime}} y_{\tau^{\prime}}-y_{b^{\prime}} y_{\tau}-3 y_{t^{\prime}} y_{\nu^{\prime}} \\
4 \pi \gamma_{b^{\prime}}^{(1)} & =-\frac{7}{15} \alpha_{1}-3 \alpha_{2}-\frac{16}{3} \alpha_{3}+6 y_{b^{\prime}}+3 y_{b}+y_{t^{\prime}}+y_{\tau}+y_{\tau^{\prime}} \\
(4 \pi)^{2} \gamma_{b^{\prime}}^{(2)} & =\frac{371}{90} \alpha_{1}^{2}+\frac{27}{2} \alpha_{2}^{2}+\frac{80}{9} \alpha_{3}^{2}+\alpha_{1} \alpha_{2}+\frac{8}{9} \alpha_{1} \alpha_{3} \\
& +8 \alpha_{2} \alpha_{3}+16 y_{b^{\prime}} \alpha 3+16 y_{b} \alpha_{3}+\frac{2}{5} y_{b^{\prime}} \alpha_{1}+\frac{4}{5} y_{t^{\prime}} \alpha_{1} \\
& -\frac{2}{5} y_{b} \alpha_{1}+\frac{6}{5} y_{\tau^{\prime}} \alpha_{1}+\frac{6}{5} y_{\tau} \alpha_{1}+6 y_{b^{\prime}} \alpha_{2} \\
& -22 y_{b^{\prime}}^{2}-9 y_{b}^{2}-3 y_{\tau^{\prime}}^{2}-3 y_{\tau}^{2}-5 y_{b^{\prime}} y_{t^{\prime}}-3 y_{b} y_{t} \\
& -y_{\tau^{\prime}} y_{\nu^{\prime}}-5 y_{t^{\prime}}^{2}-9 y_{b^{\prime}} y_{b}-3 y_{t} y_{t^{\prime}}-3 y_{b^{\prime}} y_{\tau}-3 y_{b^{\prime}} y_{\tau^{\prime}}-y_{t^{\prime}} y_{\nu^{\prime}}
\end{aligned}
$$

$$
\begin{aligned}
4 \pi \gamma_{\tau^{\prime}}^{(1)} & =-\frac{9}{5} \alpha_{1}-3 \alpha_{2}+3 y_{b^{\prime}}+3 y_{b}+y_{\tau}+4 y_{\tau^{\prime}}+y_{\nu^{\prime}} \\
(4 \pi)^{2} \gamma_{\tau^{\prime}}^{(2)} & =\frac{171}{10} \alpha_{1}^{2}+\frac{27}{2} \alpha_{2}^{2}+\frac{9}{5} \alpha_{1} \alpha_{2}+16 y_{b^{\prime}} \alpha_{3}+16 y_{b} \alpha_{3} \\
& -\frac{2}{5} y_{b^{\prime}} \alpha_{1}-\frac{2}{5} y_{b} \alpha_{1}+\frac{6}{5} y_{\tau^{\prime}} \alpha_{1}+\frac{6}{5} y_{\tau} \alpha_{1}+6 y_{\tau^{\prime}} \alpha_{2} \\
& -9 y_{b^{\prime}}^{2}-9 y_{b}^{2}-10 y_{\tau^{\prime}}^{2}-3 y_{\tau}^{2}-3 y_{b^{\prime}} y_{t^{\prime}}-3 y_{b} y_{t} \\
& -y_{\tau^{\prime}} y_{\nu^{\prime}}-3 y_{\tau} y_{\tau^{\prime}}-9 y_{\tau^{\prime}} y_{b^{\prime}}-9 y_{\tau^{\prime}} y_{b}-3 y_{\nu^{\prime}} \\
& -3 y_{\nu^{\prime}} y_{t}-3 y_{\nu^{\prime}} y_{t^{\prime}}-2 y_{\tau^{\prime}} y_{\nu^{\prime}}
\end{aligned}
$$




$$
\begin{aligned}
4 \pi \gamma_{\nu^{\prime}}^{(1)} & =-\frac{3}{5} \alpha_{1}-3 \alpha_{2}+3 y_{t^{\prime}}+3 y_{t}+4 y_{\nu^{\prime}}+y_{\tau^{\prime}} \\
(4 \pi)^{2} \gamma_{\nu^{\prime}}^{(2)} & =\frac{267}{50} \alpha_{1}^{2}+\frac{27}{2} \alpha_{2}^{2}+\frac{9}{5} \alpha_{1} \alpha_{2}+16 y_{t} \alpha_{3}+16 y_{t^{\prime}} \alpha_{3}+\frac{6}{5} y_{\tau^{\prime}} \alpha_{1}+6 y_{\nu^{\prime}} \alpha_{2} \\
& +\frac{6}{5} y_{\nu^{\prime}} \alpha_{1}+\frac{4}{5} y_{t} \alpha_{1}+\frac{4}{5} y_{t^{\prime}} \alpha_{1}-9 y_{t}^{2}-9 y_{t^{\prime}}^{2}-10 y_{\nu^{\prime}}^{2}-3 y_{b} y_{t}-3 y_{b^{\prime}} y_{t^{\prime}} \\
& -3 y_{\tau^{\prime}} y_{\nu^{\prime}}-3 y_{\tau^{\prime}}^{2}-y_{\tau} y_{\tau^{\prime}}-3 y_{\tau^{\prime}} y_{b}-3 y_{\tau^{\prime}} y_{b^{\prime}}-9 y_{\nu^{\prime}} y_{t} \\
& -9 y_{\nu^{\prime}} y_{t^{\prime}} .
\end{aligned}
$$

For the Higgs mass terms, we have:

$$
\begin{gathered}
\frac{d m_{H_{2}}^{2}}{d t}=3 \sum_{q=t, t^{\prime}} y_{q} \mathcal{U}_{q}+y_{\nu^{\prime}} \mathcal{U}_{\nu^{\prime}}-\frac{3}{5} \alpha_{1} M_{1}^{2}-3 \alpha_{2} M_{2}^{2} \\
\frac{d m_{H_{1}}^{2}}{d t}=3 \sum_{q=b, b^{\prime}} y_{q} \mathcal{D}_{q}+\sum_{l=\tau, \tau^{\prime}} y_{l} \mathcal{D}_{l}-\frac{3}{5} \alpha_{1} M_{1}^{2}-3 \alpha_{2} M_{2}^{2}
\end{gathered}
$$

The third and fourth generation scalar quark and lepton soft mass terms evolve according to:

$$
\begin{gathered}
\frac{d m_{Q, Q^{\prime}}^{2}}{d t}=y_{t, t^{\prime}} \mathcal{U}_{t, t^{\prime}}+y_{b, b^{\prime}} \mathcal{D}_{b, b^{\prime}}-\frac{1}{15} \alpha_{1} M_{1}^{2}-3 \alpha_{2} M_{2}^{2}-\frac{16}{3} \alpha_{3} M_{3}^{2} \\
\frac{d m_{U, U^{\prime}}^{2}}{d t}=2 y_{t, t^{\prime}} \mathcal{U}_{t, t^{\prime}}-\frac{16}{15} \alpha_{1} M_{1}^{2}-\frac{16}{3} \alpha_{3} M_{3}^{2} \\
\frac{d m_{D, D^{\prime}}^{2}}{d t}=2 y_{b, b^{\prime}} \mathcal{D}_{b, b^{\prime}}-\frac{4}{15} \alpha_{1} M_{1}^{2}-\frac{16}{3} \alpha_{3} M_{3}^{2} \\
\frac{d m_{L, L^{\prime}}^{2}}{d t}=y_{\tau, \tau^{\prime}} \mathcal{D}_{\tau, \tau^{\prime}}+\delta_{i L^{\prime}} y_{\nu^{\prime}} \mathcal{U}_{\nu^{\prime}}-\frac{3}{5} \alpha_{1} M_{1}^{2}-3 \alpha_{2} M_{2}^{2} \\
\frac{d m_{E, E^{\prime}}^{2}}{d t}=2 y_{\tau, \tau^{\prime}} \mathcal{D}_{\tau, \tau^{\prime}}-\frac{12}{5} \alpha_{1} M_{1}^{2} ; \frac{d m_{N}^{2}}{d t}=2 y_{\nu^{\prime}} \mathcal{U}_{\nu^{\prime}}
\end{gathered}
$$


In the above,

$$
\begin{gathered}
\mathcal{U}_{t, t^{\prime}}=m_{H_{2}}^{2}+m_{Q, Q^{\prime}}^{2}+m_{U, U^{\prime}}^{2}+A_{t, t^{\prime}}^{2} \\
\mathcal{U}_{\nu^{\prime}}=m_{H_{2}}^{2}+m_{L^{\prime}}^{2}+m_{N}^{2}+A_{\nu^{\prime}}^{2} \\
\mathcal{D}_{b, b^{\prime}}=m_{H_{1}}^{2}+m_{Q, Q^{\prime}}^{2}+m_{D, D^{\prime}}^{2}+A_{b, b^{\prime}}^{2} \\
\mathcal{D}_{\tau, \tau^{\prime}}=m_{H_{1}}^{2}+m_{L, L^{\prime}}^{2}+m_{E, E^{\prime}}^{2}+A_{\tau, \tau^{\prime}}^{2}
\end{gathered}
$$

The running of the first and second generation scalar quark and lepton soft-SUSYbreaking masses is obtained by neglecting all Yukawa couplings in the expressions above. The parameters $\mu$, specifying mixing of the Higgs superfields in the superpotential, and $B$, such that $B \mu$ is the coefficient of the $H_{1} H_{2}$ scalar field mixing term in the soft-SUSY-breaking potential, evolve according to:

$$
\begin{gathered}
\frac{d \log (\mu)}{d t}=\frac{3}{2} \sum_{q=t, t^{\prime}, b, b^{\prime}} y_{q}+\frac{1}{2} \sum_{l=\tau, \tau^{\prime}, \nu^{\prime}} y_{l}-\frac{3}{10} \alpha_{1}-\frac{3}{2} \alpha_{2} ; \\
\frac{d B}{d t}=3 \sum_{q=t, t^{\prime}, b, b^{\prime}} y_{q} A_{q}+\sum_{l=\tau, \tau^{\prime}, \nu^{\prime}} y_{l} A_{l}+\frac{3}{5} \alpha_{1} M_{1}+3 \alpha_{2} M_{2} .
\end{gathered}
$$

The running of the third and fourth family soft-SUSY-breaking potential tri-linear term $A$ coefficients is given by:

$$
\begin{gathered}
\frac{d A_{t, t^{\prime}}}{d t}=6 A_{t, t^{\prime}} y_{t, t^{\prime}}+A_{b, b^{\prime}} y_{b, b^{\prime}}+3 A_{t^{\prime}, t} y_{t^{\prime}, t}+A_{\nu^{\prime}} y_{\nu^{\prime}} \\
+\frac{13}{15} \alpha_{1} M_{1}+3 \alpha_{2} M_{2}+\frac{16}{3} \alpha_{3} M_{3} \\
\frac{d A_{b, b^{\prime}}}{d t}=6 A_{b, b^{\prime}} y_{b, b^{\prime}}+A_{t, t^{\prime}} y_{t, t^{\prime}}+3 A_{b^{\prime}, b} y_{b^{\prime}, b}+\sum_{l=\tau, \tau^{\prime}} A_{l} y_{l} \\
+\frac{7}{15} \alpha_{1} M_{1}+3 \alpha_{2} M_{2}+\frac{16}{3} \alpha_{3} M_{3} ; \\
\frac{d A_{\tau, \tau^{\prime}}}{d t}=4 A_{\tau, \tau^{\prime}} y_{\tau, \tau^{\prime}}+A_{\tau^{\prime}, \tau} y_{\tau^{\prime}, \tau}+3 \sum_{q=b, b^{\prime}} A_{q} y_{q}+\delta_{i, \tau^{\prime}} A_{\nu^{\prime}} y_{\nu^{\prime}} \\
+\frac{9}{5} \alpha_{1} M_{1}+3 \alpha_{2} M_{2} ;
\end{gathered}
$$




$$
\frac{d A_{\nu^{\prime}}}{d t}=4 A_{\nu^{\prime}} y_{\nu^{\prime}}+A_{\tau^{\prime}} y_{\tau^{\prime}}+3 \sum_{q=t, t^{\prime}} A_{q} y_{q}+3 \alpha_{2} M_{2}+\frac{3}{5} \alpha_{1} M_{1}
$$

The Higgs boson, scalar quark and scalar lepton mass-squared RGE's generally include a term in each equation that is a numerical factor times $\alpha_{1} \mathcal{S}^{\prime}$, where

$$
\begin{aligned}
\mathcal{S}^{\prime} & \equiv m_{H_{2}}^{2}-m_{H_{1}}^{2}+m_{Q}^{2}+m_{Q^{\prime}}^{2}-2 m_{U}^{2}+m_{D}^{2} \\
& -2 m_{U^{\prime}}^{2}+m_{D^{\prime}}^{2}-m_{L}^{2}-m_{L^{\prime}}^{2}+m_{E}^{2}+m_{E^{\prime}}^{2}
\end{aligned}
$$

Substituting from the RGE's with $\mathcal{S}^{\prime}$ terms included, one finds

$$
\frac{d \mathcal{S}^{\prime}}{d t}=\frac{46}{5} \alpha_{1} \mathcal{S}^{\prime}
$$

Because of the universal scalar mass boundary condition at $M_{U}$ that we assume, $\mathcal{S}^{\prime}\left(M_{U}\right)=0$. Therefore $\mathcal{S}^{\prime}(t)=0$ for all $t$ and we do not include this term in the mass-squared RGE's presented above.

The final soft-SUSY-breaking parameters are the gaugino masses. Using the notation $N_{g}$ for the number of generations, these evolve according to:

$$
\begin{aligned}
& \frac{d M_{1}}{d t}=\left(2 N_{g}+\frac{3}{5}\right) \alpha_{1} M_{1} ; \\
& \frac{d M_{2}}{d t}=\left(2 N_{g}-5\right) \alpha_{2} M_{2} \\
& \frac{d M_{3}}{d t}=\left(2 N_{g}-9\right) \alpha_{3} M_{3}
\end{aligned}
$$

Finally, we present the gauge coupling RGE's for completeness. These are the same as the massless fourth generation neutrino case, since the extra right handed neutrino that provides a mass term in our case is decoupled from the gauge sector. Keeping the number of generations, $N_{g}$, explicit, including the third and fourth (unmixed) generation Yukawa couplings, and including exactly two Higgs doublets (as appropriate for the MSSM), one has the gauge RGE's:

$$
\begin{aligned}
\frac{d \alpha_{1}}{d t} & =\left(2 N_{g}+\frac{3}{5}\right) \alpha_{1}^{2}+\frac{\alpha_{1}^{2}}{4 \pi}\left[\left(\frac{38}{15} N_{g}+\frac{9}{25}\right) \alpha_{1}+\left(\frac{6}{5} N_{g}+\frac{9}{5}\right) \alpha_{2}+\frac{88}{15} N_{g} \alpha_{3}\right. \\
& \left.-\frac{18}{5}\left(y_{\tau}+y_{\tau^{\prime}}\right)-\frac{14}{5}\left(y_{b}+y_{b^{\prime}}\right)-\frac{26}{5}\left(y_{t}+y_{t^{\prime}}\right)\right]
\end{aligned}
$$




$$
\begin{gathered}
\frac{d \alpha_{2}}{d t}=\left(2 N_{g}-5\right) \alpha_{2}^{2}+\frac{\alpha_{2}^{2}}{4 \pi}\left[\left(\frac{2}{5} N_{g}+\frac{3}{5}\right) \alpha_{1}+\left(14 N_{g}-17\right) \alpha_{2}+8 N_{g} \alpha_{3}\right. \\
\left.-2\left(y_{\tau}+y_{\tau^{\prime}}\right)-6\left(y_{b}+y_{b^{\prime}}+y_{t}+y_{t^{\prime}}\right)\right] \\
\frac{d \alpha_{3}}{d t}=\left(2 N_{g}-9\right) \alpha_{3}^{2}+\frac{\alpha_{3}^{2}}{4 \pi}\left[\frac{11}{15} N_{g} \alpha_{1}+3 N_{g} \alpha_{2}+\left(\frac{68}{3} N_{g}-54\right) \alpha_{3}\right. \\
\left.-4\left(y_{b}+y_{b^{\prime}}+y_{t}+y_{t^{\prime}}\right)\right]
\end{gathered}
$$

The renormalization group equations were implemented numerically, and iteration was employed to find a fully consistent solution for the complete evolution between $M_{U}$ and $m_{Z}$.

\section{APPENDIX}

In this appendix we discuss the question of the accuracy with which $\cos 2 \beta$ and, thence, $\tan \beta$, can be determined using the mass differences of Eq. $(10){ }^{[42]}$

To use $m_{\widetilde{\ell}_{L}}^{2}-m_{\widetilde{\nu}}^{2}$ requires accurate determinations of $m_{\widetilde{\nu}}$ and $m_{\widetilde{\ell}_{L}}$. The required measurements are best performed at an $e^{+} e^{-}$collider where the center-of-mass energy is precisely known and the energy spectra of final leptons can then be directly related to the masses of the produced particles and their decay products. Determination of $m_{\tilde{\ell}_{L}}$ is relatively straightforward since $\widetilde{\ell}_{L} \rightarrow l \widetilde{\chi}_{1}^{0}$ is almost always an important, if not dominant, mode - if $m_{\widetilde{\ell}_{L}}>m_{\widetilde{\chi}_{1}^{+}} \sim m_{\widetilde{\chi}_{2}^{0}}$, then $\widetilde{\ell}_{L} \rightarrow \widetilde{\chi}_{1}^{+} \nu$ and $\tilde{\ell}_{L} \rightarrow \widetilde{\chi}_{2}^{0} \ell$ can be competitive. A fit to the $\ell$ spectrum will yield the masses of both the $\widetilde{\ell}_{L}$ and the $\tilde{\chi}_{1}^{0}$. Should the $\tilde{\chi}_{1}^{+} \nu$ and $\tilde{\chi}_{2}^{0} \ell$ modes also be important, the fit would presumably reveal the presence of several upper end points, and allow determination of both $m_{\widetilde{\chi}_{1}^{0}}$ and $m_{\widetilde{\chi}_{2}^{0}}$. A Monte Carlo study is desirable to determine the exact accuracy with which all masses could be determined in the multiple decay case. Perhaps accuracies of about $\pm 10 \mathrm{GeV}$ could be achieved. For $m_{\widetilde{\ell}_{L}} \sim 240 \mathrm{GeV}$ (near our lower limit in the $N_{g}=4$ case scenarios, but already rather marginal for a $\sqrt{s}=500 \mathrm{GeV}$ NLC) this would correspond to accuracies of $\sim 4 \%$, i.e. on the edge of what would be useful.

Determination of $m_{\widetilde{\nu}}$ follows a similar pattern. We give below discussions for two scenarios: a) $m_{\widetilde{\nu}}<m_{\widetilde{\chi}_{1}^{+}}$and b) $m_{\widetilde{\nu}}>m_{\widetilde{\chi}_{1}^{+}}$. Scenario a) does not arise for the specific $N_{g}=4$ models illustrated in Figs. 13-15, but might be relevant if there was significant non-universality in the $M_{U^{-S c a l e}}$ soft-SUSY-breaking parameters. For $N_{g}=4$, universal $m_{0}$ and $M_{i}$ values always lead to $m_{\widetilde{\nu}}>m_{\widetilde{\chi}_{1}^{+}}$, as shown. 
If $m_{\widetilde{\nu}}<m_{\widetilde{\chi}_{1}^{+}}$then the $\widetilde{\chi}_{1}^{+}$decays to a mixture of $\widetilde{\ell}_{L} \nu$ and $\ell \widetilde{\nu}$; since $m_{\widetilde{\ell}_{L}}>m_{\widetilde{\nu}}$ the latter decay is usually the dominant one - indeed, in many cases $m_{\widetilde{\ell}_{L}}-m_{\widetilde{\nu}}$ is large enough that the $\widetilde{\chi}_{1}^{+} \rightarrow \widetilde{\ell}_{L} \nu$ channel is forbidden even though the $\widetilde{\chi}_{1}^{+} \rightarrow \ell \widetilde{\nu}$ channel is open. At an $e^{+} e^{-}$collider the end-points of the $\ell$ spectrum from $\tilde{\chi}_{1}^{+} \widetilde{\chi}_{1}^{-}$ production followed by $\widetilde{\chi}_{1}^{ \pm} \rightarrow \ell \widetilde{\nu}$ decay provide a determination of both $m_{\widetilde{\chi}_{1}^{+}}$and $m_{\widetilde{\nu}}$; direct observation of the $\widetilde{\nu}$ is not required, a fortunate fact given that it decays invisibly to $\nu \widetilde{\chi}_{1}^{0}$.

If $m_{\widetilde{\nu}}>m_{\widetilde{\chi}_{1}^{+}}$, then $\widetilde{\nu} \rightarrow \tilde{\chi}_{1}^{+} \ell$, and the $\tilde{\chi}_{1}^{+}$decays via a virtual sneutrino or slepton to the three-body mode $\ell \nu \widetilde{\chi}_{1}^{0}$, via virtual squark to $q \bar{q} \widetilde{\chi}_{1}^{0}$, or via virtual or real $W$ to a mixture of these two final states. Analogous to the previous case, the $\ell$ spectrum end-points will allow determination of $m_{\widetilde{\nu}}$ and $m_{\widetilde{\chi}_{1}^{+}}$, although one will have to carefully account for the underlying smooth $\ell$ spectrum from the $\widetilde{\chi}_{1}^{+}$decay if events containing only $2 \ell+4 j+$ missing energy (which avoid contamination from the soft $\ell(\mathrm{s})$ from $\widetilde{\chi}_{1}^{ \pm}$decay) do not occur at a sufficient rate. Ideally, it would be good to learn first about the $\tilde{\chi}_{1}^{+} \widetilde{\chi}_{1}^{-}$channel by setting $\sqrt{s}$ to a value above $2 m_{\widetilde{\chi}_{1}^{ \pm}}$ but below (or not far above) $2 m_{\widetilde{\nu}}$ - due to the rapid turn-on of a two-fermion channel, the rate for $\widetilde{\chi}_{1}^{+} \widetilde{\chi}_{1}^{-}$production might be reasonable, while the $\widetilde{\nu} \widetilde{\nu}$ rate could be zero or quite small (due to a large $m_{\widetilde{\nu}}-m_{\widetilde{\chi}_{1}^{+}}$splitting and/or the slow turn-on of the $\widetilde{\nu} \widetilde{\nu}$ spin-0 pair channel). To study $\widetilde{\nu} \widetilde{\nu}$, one would then up the energy to a level such that the $\widetilde{\nu} \widetilde{\nu}$ rate was large.

A significant complication is the fact that $\tilde{\ell}_{L} \tilde{\ell}_{L}$ production would also be present at a similar rate to $\widetilde{\nu} \widetilde{\nu}$ (recall that $m_{\widetilde{\ell}_{L}} \gtrsim m_{\widetilde{\nu}}$ ). For the present case of $m_{\widetilde{\nu}}>m_{\widetilde{\chi}_{1}^{ \pm}}, \widetilde{\ell}_{L} \rightarrow \ell \widetilde{\chi}_{1}^{0}, \nu \widetilde{\chi}_{1}^{+}, \ell \widetilde{\chi}_{2}^{0}$ will all occur, most probably with similar branching ratios. Events in which both $\widetilde{\ell}_{L}$ 's decay to $\ell \widetilde{\chi}_{1}^{0}$ can be eliminated by requiring some jet activity (which is generally present for the $\widetilde{\nu} \widetilde{\nu}$ final states). If both $\widetilde{\ell}_{L}$ 's decay to $\widetilde{\chi}_{1}^{ \pm} \nu$, then the only charged $\ell$ 's would be from decays of the $\widetilde{\chi}_{1}^{ \pm}$'s, which would yield a smooth (and relatively soft) $\ell$ spectrum; in addition, these $\widetilde{\ell}_{L} \widetilde{\ell}_{L}$ events would tend to have larger missing energy than the $\widetilde{\nu} \widetilde{\nu}$ events. If one $\widetilde{\ell}_{L}$ decays to $\ell \widetilde{\chi}_{1}^{0}$ and the other to $\nu \widetilde{\chi}_{1}^{+}$, followed by $\widetilde{\chi}_{1}^{+} \rightarrow \ell \nu \widetilde{\chi}_{1}^{0}$, then we could end up with a $2 \ell+2 j+$ missing energy final state that might not be easily distinguished from the $2 \ell+4 j+$ missing energy state of interest for $\widetilde{\nu} \widetilde{\nu}$ events (certainly not all jets in the latter case would be detectable). But, the spectrum of the $\ell$ from the $\widetilde{\chi}_{1}^{+}$decay would be soft and smooth, so that the $\ell$-spectrum thresholds present in $\widetilde{\nu} \rightarrow \ell \widetilde{\chi}_{1}^{ \pm}$might still stand out.

Thus, it would seem that there are means for isolating the $\widetilde{\nu} \widetilde{\nu}$ events of interest and extracting the $\ell$ spectrum thresholds that would allow a reasonable determination of $m_{\widetilde{\nu}}$ (especially if $m_{\widetilde{\chi}_{1}^{ \pm}}$is known from a lower energy measurement). Unfortunately, these complicated scenarios have not been explicitly studied for 
an $e^{+} e^{-}$collider. The results for simpler scenarios (see Ref. [30] and references therein) suggest the crude estimate that mass determinations for $m_{\widetilde{\nu}}$ might be possible within $\pm 5 \%$, at least for masses of order $200 \mathrm{GeV}$ and below (i.e. comparable to the minimum possible $m_{\widetilde{\nu}}$ values in the scenarios discussed in the previous sub-section.) However, including the fact that the $m_{\widetilde{\ell}_{L}}$ and $m_{\widetilde{\nu}}$ errors must be combined in quadrature, despite this fairly small uncertainty in the mass measurements we would not have the accuracy required for a $3 \sigma$ sensitivity to $\cos 2 \beta$, even for the smallest possible masses allowed in the $N_{g}=4$ case.

Let us now turn to the $m_{\widetilde{d}_{L}}^{2}-m_{\widetilde{u}_{L}}^{2}$ measurement. First, there is a very real possibility (a near certainty for $N_{g}=4$ ) that the squarks are simply so heavy, $m_{\widetilde{q}} \gtrsim 300 \mathrm{GeV}$, that impossible accuracy would be required for sensitivity to $\cos 2 \beta$. Even for masses below this level, it is still far from clear that the required accuracy can be achieved. Squarks will decay to a quark plus real or virtual gluino, the latter then decaying to a variety of final states (with $q \bar{q} \widetilde{\chi}_{1}^{0}$ unlikely to be dominant for the larger $m_{\widetilde{g}}$ predicted for $N_{g}=4$ ). Jet spectra end-points (which would appear on top of a smooth jet-spectrum background from the real or virtual $\widetilde{g}$ decays) could provide a certain level of accuracy in the squark mass determination, but there would be a lot of overlap of the spectra from different $\widetilde{g}$ decay channels, and of the spectra from the $\widetilde{d}$ and $\widetilde{u}$ decays themselves, that might very well prevent a good determination of $m_{\tilde{d}}-m_{\tilde{u}}$. An extremely careful Monte Carlo study is required to be able to determine with any certainty the level of accuracy that can be achieved.

Overall, we conclude that our ability to determine $\cos 2 \beta$ and thence $\tan \beta$ must remain a topic of further study. Fortunately, the uncertainty in $\cos 2 \beta$ is not the limiting factor determining the level of accuracy with which other mass sum rules and relations can be tested. Other experimental and theoretical uncertainties are almost certainly more important. 


\section{REFERENCES}

1. J.F. Gunion, D.W. McKay and H. Pois, Phys. Lett. B334, (1994) 339.

2. For a detailed discussion of the neutrino sector, see S. King, Phys. Lett. B281, 295 (1992).

3. OPAL Collaboration, M.Z. Akrawy et al., Phys. Lett. B236, 364 (1990); ALEPH Collaboration, D. Decamp et al., ibid., 236, 511 (1990). A recent survey of lower bounds on heavy and neutral charged leptons and on heavy quarks is given by the Particle Data Group, K. Hikasa et al., Phys. Rev. D50, Part 1 (1994).

4. M. Drees and S.P. Martin, 'Implications of SUSY Model Building', Report of Subgroup 2 of the DPF Working Group on "Electroweak Symmetry Breaking and Beyond the Standard Model", MADPH-95-879.

5. H. Baer, J. Gunion, C. Kao and H. Pois, Phys. Rev. D51, 2159 (1995).

6. An exception is the early $N_{g}=4, m_{\nu^{\prime}}=0$ supergravity extension of the SM by M. Cvetic and C. Preitschopf, Nucl. Phys. B272, 490 (1986).

7. For more recent work, see P. Agrawal and W. -S. Hou, Phys. Rev. D46, 1022 (1992); P. Agrawal, S. Ellis and W. -S. Hou, Phys. Lett. B256, 289 (1991); S. King, Ref. [2]; C. Hill and E. Paschos, ibid., 241, 96 (1990); W.-S. Hou and R. G. Stuart, Nucl. Phys. B349, 91 (1991); M. Sher and Y. Yuan, Phys. Lett. B285, 336 (1992); H. Fritzsch, Phys. Lett. B289, 92 (1992); B. Mukhopadhyaya and D.P. Roy, Phys. Rev. D48, 2105 (1993); J.L. Hewett, SLAC-PUB-6521 (1994); Z. Berezhiani and E. Nardi, UM-TH-94-36, hep-ph/9411249 (1994).

8. Mark II Collaboration, G. S. Abrams et al., Phys. Rev. Lett. 63, 2173 (1989); L3 Collaboration, B. Advera et al., Phys. Lett. B231, 509 (1989); OPAL Collaboration, I. Decamp et al., ibid., 231, 519 (1989); DELPHI Collaboration, M. Z. Akrawy et al., ibid., 231, 539 (1989).

9. For a recent example of theoretical motivation of $N_{g}=4$, see Berezhiani and Nardi, Ref. [7].

10. P. Langacker, in Proceedings of the PASCOS-90 Symposium, eds. P. Nath and S. Reucroft (World Scientific, 1990); P. Langacker and M. -X. Luo, Phys. Rev. D44, 817 (1991); U. Amaldi, W. de Boer, and H. Furstenau, Phys. Lett. B260, 447 (1991); J. Ellis, S. Kelley and D. V. Nanopoulos, ibid. 260, 131 (1991).

11. P. Langacker and N. Polonsky, Phys. Rev. D47, 4028 (1993); ibid., 49, 1454 (1994); V. Barger, M. Berger, P. Ohmann, and R. J. N. Phillips, Phys. Lett. B314, 351 (1993); G. Kane, C. Kolda, L. Roszkowski, and J. Wells, Phys. Rev. D49, 6173 (1994); G. Ross and R.G. Roberts, Nucl. Phys. B377, 571 (1992); B.D. Wright, MAD/PH/812. For string Yukawa threshold effects, see I. Antoniadis, E. Gava, K.S. Narain and T.R. Taylor, Nucl. Phys. B407, 706 (1993). 
12. P.H. Frampton, S. Nandi and J.G. Scanio, Phys. Lett. B85, 225 (1979).

13. S. Dimopoulos and A. Pomarol, CERN-TH/95-44, hep-ph/9502397.

14. H.E. Haber, preprint CERN-TH/95-178 (1995).

15. D. V. Nanopoulos and D. Ross, Phys. Lett. B118, 99 (1982); J. Bjorkman and D. Jones, Nucl. Phys. B259, 533 (1985).

16. P. Langacker and N. Polonsky, UPR-0642T

17. M. L. Swartz, SLAC Report No. PUB-6710

18. J. Bagger, V. Matchev and D. Pierce, Phys. Lett. B348 443 (1995).

19. See, for example, V. Barger, M. S. Berger and P. Ohmann, Phys. Rev. D47, 1093 (1993).

20. F. Abe et al. (CDF Collaboration), Phys. Rev. Lett. 74, 2626 (1995); S. Abachi et al. (D0 Collaboration), Phys. Rev. Lett. 74, 2632 (1995.

21. B. Pendelton and G. Ross, Phys. Lett. B98, 291 (1981); C. Hill, Phys. Rev. D24, 691 (1981); W. Bardeen, M. Carena, S. Pokorski and C. Wagner, Phys. Lett. B320, 110 (1994); J. Bagger, S. Dimopoulos and E. Masso, Phys. Rev. Lett. 55, 920 (1985).

22. See, for example, G. Gamberini, G. Ridolfi and F. Zwirner, Nucl. Phys. B331 331 (1990).

23. T.L. Barklow et al., 'Electroweak Symmetry Breaking and Beyond the Standard Model', SLAC-PUB-95-6893 (1995).

24. J.F. Gunion, A. Stange and S. Willenbrock, in preparation.

25. S. Mrenna and G. Kane, CALT-68-1938 (1994).

26. These effects were first explored in J.F. Gunion and S. Geer, Proceedings of the "Workshop on Physics at Current Accelerators and the Supercollider", eds. J. Hewett, A. White, and D. Zeppenfeld, Argonne National Laboratory, 2-5 June (1993), ANL-HEP-CP-93-92, p. 335.

27. M. Drees and M.M. Nojiri, Phys. Rev. D45, 2482 (1992).

28. S. Martin and P. Ramond, Phys. Rev. D48, 5365 (1993), provide a convenient summary of squark and slepton mass relations in the $N_{g}=3$ case.

29. J.F. Gunion and H.E. Haber, Phys. Rev. D37, 2515 (1988).

30. See H. Baer et al., "Low Energy Supersymmetry Phenomenology", preprint FSU-HEP-950401, and references therein.

31. V. Barger, M. Berger, J. Gunion and T. Han, preprint UCD-95-12 (1995).

32. F. Abe et al., Phys. Rev. Lett. 64, 147 (1990), Phys. Rev. Lett. 68, 447 (1992); T. Trippe, Particle Data Group, private communication.

33. J. Houston and R. Blair, private communication. 
34. W.-S. Hou and R. Stuart, Phys. Rev. D43, 3669 (1991).

35. D. Schaile, talk presented at the 27th Int. Conf. on High Energy Physics,, Glasgow, Scotland, July 20-27, 1994; SLD Collaboration, F. Abe et al., Phys. Rev. Lett. 73, 25 (1994).

36. M. Peskin and T. Takeuchi, Phys. Rev. D46, 381 (1992).

37. C. P. Burgess, S. Godfrey, H. Konig, D. London and I. Maksymyk, Phys. Rev. D49, 6115 (1994); C. P. Burgess, S. Godfrey, H. Konig, D. London and I. Maksymyk, Phys. Lett. B326, 276 (1994); I. Maksymyk, C. P. Burgess and D. London, Phys. Rev. D50, 529 (1994); P. Bamert, C.P.Burgess MCGILL94-27, (1994).

38. A. Kundu and P. Roy, SINP-TNP/94-07, TIFR/TH/94-38.

39. H. Pois, T.C. Yuan, unpublished

40. Figures available on the CDF WWW Home Page and private communications.

41. S. Martin and M. Vaughn, Phys. Rev. D50, 2282 (1994).

42. We thank H. Murayama for several conversations on various aspects of the discussion in this Appendix. See also Ref. 30.

\section{FIGURE CAPTIONS}

1) Gauge coupling unification, including two-loop gauge contributions but not two-loop Yukawa contributions; dashed (solid) curves correspond to $N_{g}=$ 3 (4). The illustration is for $\sin ^{2} \theta_{W}=0.2316$ and $\alpha_{e m}^{-1}\left(m_{Z}\right)=127.9$.

2) We give the allowed $m_{t^{\prime}}, m_{b^{\prime}}$ parameter space regions for $m_{t}\left(m_{t}\right)=165 \mathrm{GeV}$ in the cases $\tan \beta=1.5$ and 2.2. We have taken $m_{\nu^{\prime}}=m_{\tau^{\prime}}=50 \mathrm{GeV}$. Full two-loop contributions to the gauge coupling beta functions are included. Small dots indicate regions of $m_{t^{\prime}}, m_{b^{\prime}}$ disallowed by demanding perturbative Yukawas $\left(\lambda_{i} \leq 3.3\right)$ and gauge coupling unification to within 2.5\%. Small squares indicate additional points excluded if gauge unification is required to better than $0.01 \%$.

3) Contours in $m_{b^{\prime}}$ and $m_{t^{\prime}}$ (running mass) parameter space of constant $M_{U}$ and $\alpha_{3}\left(m_{Z}\right)$ after the inclusion of Yukawa terms in the two-loop gauge coupling beta functions, assuming $m_{t}\left(m_{t}\right)=165 \mathrm{GeV}$ and $\tan \beta=1.5$. We have taken $m_{\nu^{\prime}}=m_{\tau^{\prime}}=50 \mathrm{GeV}$.

4) We display contours of constant $\lambda_{b^{\prime}}, \lambda_{t^{\prime}}$ and $\lambda_{\tau^{\prime}}$ at $M_{U}$ in the region of $m_{t^{\prime}}, m_{b^{\prime}}$ parameter choices allowed for $2.5 \%$ unification accuracy with $m_{t}\left(m_{t}\right)=$ $165 \mathrm{GeV}$ and $\tan \beta=1.5$ or 2.2 . We have taken $m_{\nu^{\prime}}=m_{\tau^{\prime}}=50 \mathrm{GeV}$. Two-loop contributions to the gauge coupling beta functions are included.

5) In a) we illustrate the evolution of the soft-SUSY-breaking scalar masssquared parameters $m_{H_{1}}^{2}$ and $m_{H_{2}}^{2}$ as well as the value of $|\mu|$. In b) we show 
the evolution of the coefficients $m_{1}^{2}, m_{2}^{2}$ and $m_{3}^{2}$ of the $H_{1}^{2}, H_{2}^{2}$ and $2 H_{1} H_{2}$ scalar field potential terms, as well as that of $S \equiv m_{1}^{2} m_{2}^{2}-m_{3}^{4}$. We have taken $\xi_{0}=1 / \sqrt{3}, \xi_{A}=-1, m_{1 / 2}=400 \mathrm{GeV}, \tan \beta=1.5, m_{t^{\prime}}=100 \mathrm{GeV}$, $m_{b^{\prime}}=100 \mathrm{GeV}$, and $m_{\tau^{\prime}}=m_{\nu^{\prime}}=50 \mathrm{GeV}$. All masses are given in units of $m_{1 / 2}$.

6) Contours of $\mu, m_{A^{0}}$ and $m_{h^{0}}$ in the $m_{t^{\prime}}, m_{b^{\prime}}$ parameter plane for $\tan \beta=1.5$, $m_{t}\left(m_{t}\right)=165 \mathrm{GeV}$ and $m_{1 / 2}=600 \mathrm{GeV}$. We give results for the $M_{U}$-scale dilaton boundary conditions: $\xi_{0}=1 / \sqrt{3}, \xi_{A}=-1$. The diamonds indicate regions of parameter space disallowed because $m_{\widetilde{\tau}_{1}^{\prime}}<\max \left\{45 \mathrm{GeV}, m_{\widetilde{\chi}_{1}^{0}}\right\}$ at energy scale $m_{Z}$.

7) We plot the ratio of $N_{g}=4$ to $N_{g}=3$ values for: $\Gamma(h \rightarrow g g), \Gamma(h \rightarrow \gamma \gamma)$, and $\Gamma(h \rightarrow g g) \times B R(h \rightarrow \gamma \gamma)$. We adopt the scenario of $\tan \beta=1.5$, $m_{t}=165 \mathrm{GeV}, m_{t^{\prime}}=m_{b^{\prime}}=100 \mathrm{GeV}, m_{\tau^{\prime}}=m_{\nu^{\prime}}=50 \mathrm{GeV}$, and assume superpartners are sufficiently heavy that their contributions to these one-loop quantities are small.

8) Evolution of $m_{\widetilde{\tau}_{R}^{\prime}}^{2}, m_{\widetilde{\tau}_{R}}^{2}$, and $m_{\widetilde{\nu}_{R}^{\prime}}^{2}$ (in units of $m_{1 / 2}^{2}$ ). We have chosen $\tan \beta=$ 1.5, $\xi_{0}=1 / \sqrt{3}, \xi_{A}=-1$, and $m_{1 / 2}=600 \mathrm{GeV}$. Results for $m_{t^{\prime}}=100 \mathrm{GeV}$ and $m_{b^{\prime}}=90 \mathrm{GeV}$ are compared to those for $m_{t^{\prime}}=100 \mathrm{GeV}$ and $m_{b^{\prime}}=$

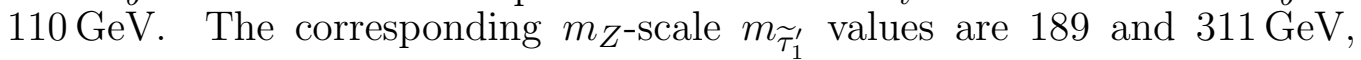
respectively.

9) We display the $\widetilde{\tau}_{1}^{\prime}$ constraint boundaries for a variety of $M_{U}$-scale scenarios. Regions to the left of the boundaries are disallowed. We have taken $m_{t}=$ $165 \mathrm{GeV}, m_{\tau^{\prime}}=m_{\nu^{\prime}}=50 \mathrm{GeV}$, and $\tan \beta=1.5$.

10) We plot the minimum value of $\xi_{0}$ that is allowed by the constraint $m_{\widetilde{\tau}_{1}^{\prime}}>$ $\max \left\{45 \mathrm{GeV}, m_{\widetilde{\chi}_{1}^{0}}\right\}$ for a given value of $m_{1 / 2}$ after scanning over all possible values of $A$, assuming running masses $m_{t}=165 \mathrm{GeV}, m_{\tau^{\prime}}=m_{\nu^{\prime}}=50 \mathrm{GeV}$, $m_{b^{\prime}}=m_{t^{\prime}}=100 \mathrm{GeV}$, and $\tan \beta=1.5$.

11) We plot the minimum possible value of $m_{\widetilde{g}}\left(m_{\tilde{g}}\right)$, and the corresponding values of $m_{h^{0}}$ and $m_{b^{\prime}}($ pole $)-m_{h^{0}}-m_{b}$, as a function of $\xi_{A}$ for a series of $\xi_{0}$ values: $\xi_{0}=0$ (solid); $\xi_{0}=0.5$ (long dashes); $\xi_{0}=1$ (dots); $\xi_{0}=2$ (dot-dash); $\xi_{0}=3$ (short dashes); $\xi_{0}=5$ (dash-dot-dot). We have taken $m_{t}\left(m_{t}\right)=$ $165 \mathrm{GeV}, m_{b^{\prime}}\left(m_{b^{\prime}}\right)=m_{t^{\prime}}\left(m_{t^{\prime}}\right)=100 \mathrm{GeV}$ (corresponding to pole masses of approximately $175 \mathrm{GeV}$ and $105 \mathrm{GeV}$ ). The minimum $m_{\tilde{g}}$ is that allowed by the $\widetilde{\tau}_{1}^{\prime}$ constraint for a given $\xi_{0}, \xi_{A}$ choice. The corresponding $\tan \beta$ value is either 1.5 or 1.6 in all cases. At each $\xi_{0}$ and $\xi_{A}$ value, all values of $m_{1 / 2} \leq$ $10 \mathrm{TeV}$ were scanned. Curves terminate when no consistent solution is found.

12) Mass spectra for the $\widetilde{\tau}_{1}^{\prime}$ for the three standard $\left(\xi_{0}, \xi_{A}\right)$ choices. At the lower values of $m_{\tilde{g}}$ the spectrum terminates either because $m_{\widetilde{\tau}_{1}^{\prime}}$ falls below $45 \mathrm{GeV}$ (high- $m_{0}$ scenario), or because $m_{\widetilde{\tau}_{1}^{\prime}}<m_{\widetilde{\chi}_{1}^{0}}$ (dilaton and no-scale scenarios). 
We have scanned in $\tan \beta$, fixing $m_{t}\left(m_{t}\right)=165 \mathrm{GeV}, m_{\nu^{\prime}}=m_{\tau^{\prime}}=50 \mathrm{GeV}$ and $m_{t^{\prime}}=m_{b^{\prime}}=100 \mathrm{GeV}$.

13) Mass spectra for dilaton boundary conditions. Masses are given in units of $m_{\tilde{g}}$. We have taken $m_{\tau^{\prime}}=m_{\nu^{\prime}}=50 \mathrm{GeV}, m_{t^{\prime}}=m_{b^{\prime}}=100 \mathrm{GeV}, \mu>0$, and scanned in $\tan \beta$.

14) Mass spectra for no-scale boundary conditions.

15) Mass spectra for the large $m_{0}$ boundary condition case.

16) A plot of $r_{\widetilde{\ell}_{L}}$ and $r_{\widetilde{\ell}_{R}}$ (see text) as a function of $\xi_{0}$ for the typical case of $m_{\widetilde{\chi}_{1}^{0}}=117 \mathrm{GeV}$. We have taken $\tan \beta=1.5, m_{t}=165 \mathrm{GeV}$, and, in the $N_{g}=4$ case, $m_{t^{\prime}}=m_{b^{\prime}}=100 \mathrm{GeV}, m_{\tau^{\prime}}=m_{\nu^{\prime}}=50 \mathrm{GeV}$.

17) We plot the maximum value of $V_{t^{\prime} b}$ for which $\Gamma\left(t^{\prime} \rightarrow b^{\prime} W^{*}\right)>\Gamma\left(t^{\prime} \rightarrow b W\right)$ as a function of $m_{t^{\prime}}$ for fixed values of $m_{b^{\prime}}=80 \mathrm{GeV}$ and $100 \mathrm{GeV}$. All masses are pole masses.

18) We show the $95 \%$ C.L. and $90 \%$ C.L. constraints on the $m_{b^{\prime}}\left(m_{b^{\prime}}\right), m_{t^{\prime}}\left(m_{t^{\prime}}\right)$ parameter space (assuming $m_{\tau^{\prime}}=m_{\nu^{\prime}}=50 \mathrm{GeV}$ and $\tan \beta=1.5$ ) from $Z$-pole precision electroweak measurements (coupled with $m_{W} / m_{Z}$ ). We consider two reference SM's specified by $m_{t}\left(m_{t}\right)=165 \mathrm{GeV}, m_{H}=100 \mathrm{GeV}$, and the two values, $\alpha_{s}\left(m_{Z}\right)=0.12$ (dashed lines) and 0.13 (solid lines), see text.

19) We plot the percentage enhancement $R_{\alpha_{s}}$ (see text) as a function of $Q$ for $N_{g}=4$ choices of $M_{S U S Y}^{Q C D}=200 \mathrm{GeV}$ and $M_{S U S Y}^{Q C D}=400 \mathrm{GeV}$. 


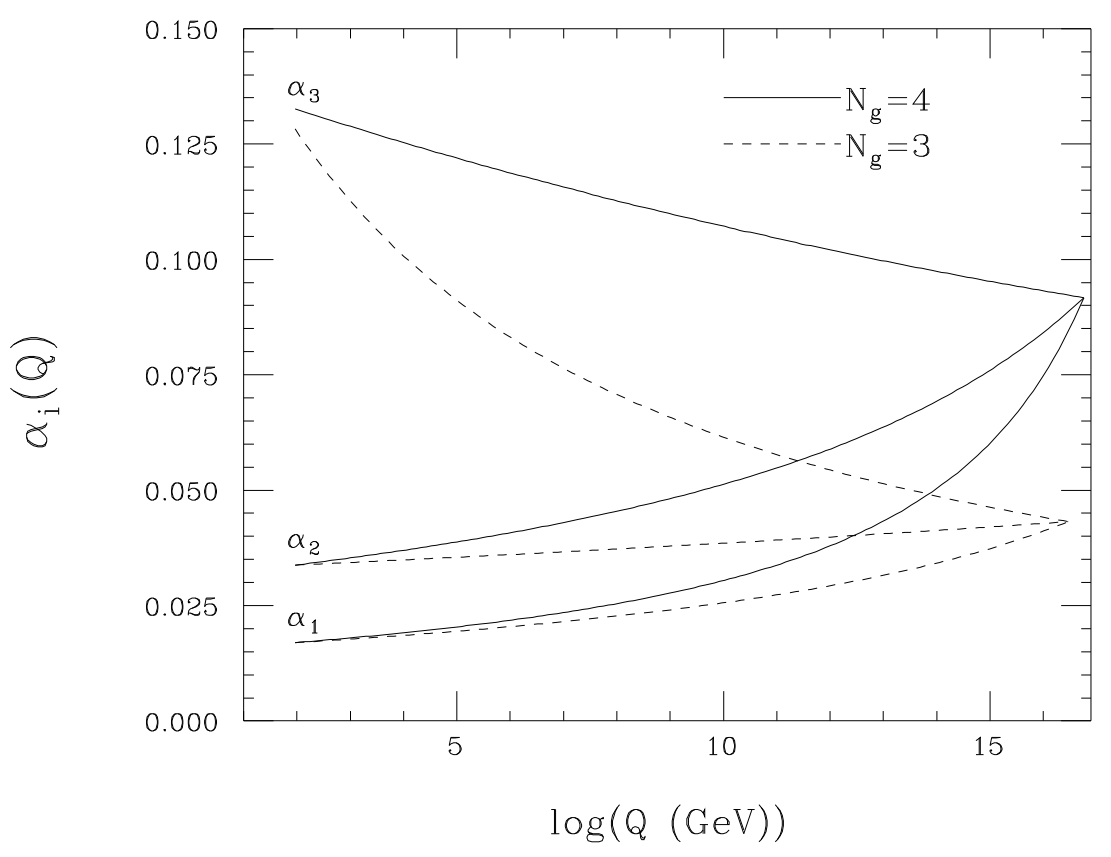

Fig. 1

Excluded Regions: $\mathrm{m}_{\mathrm{t}}\left(\mathrm{m}_{\mathrm{t}}\right)=165 \mathrm{GeV}, \mathrm{m}_{\mathrm{b}}\left(\mathrm{m}_{\mathrm{b}}\right)=4.6 \mathrm{GeV}$

Unification to within $2.5 \%(.01 \%): \cdots(\cdot+\square \square)$

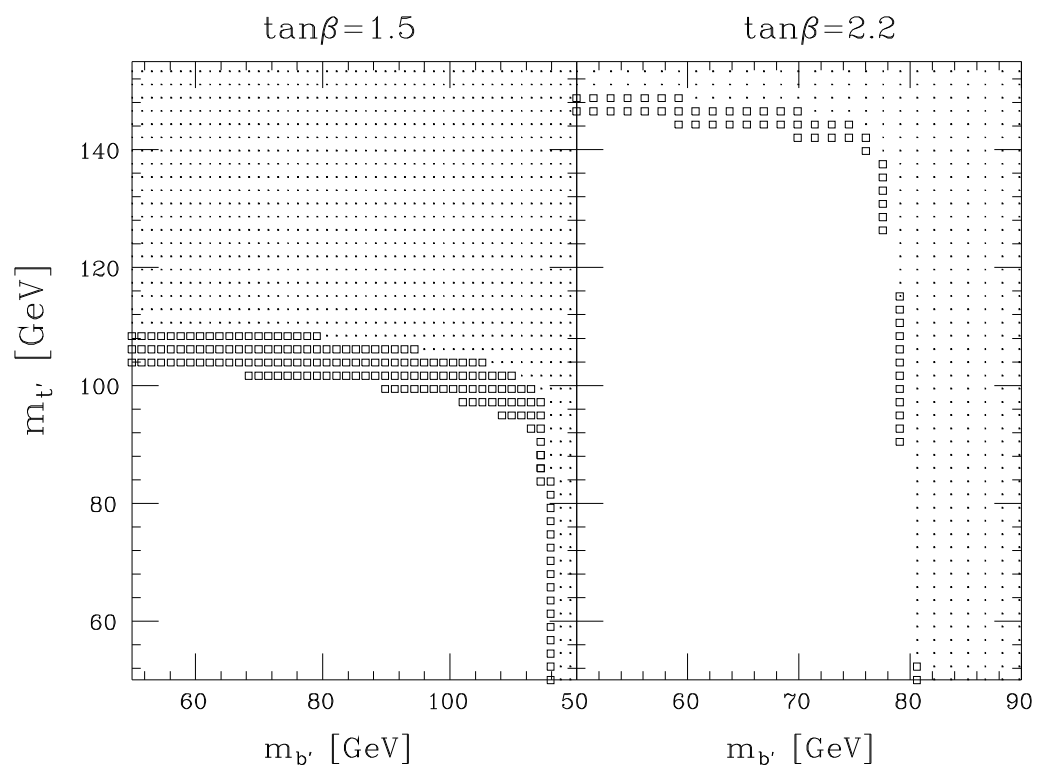

Fig. 2 
Contours: 2-Loop Yukawa Terms Included

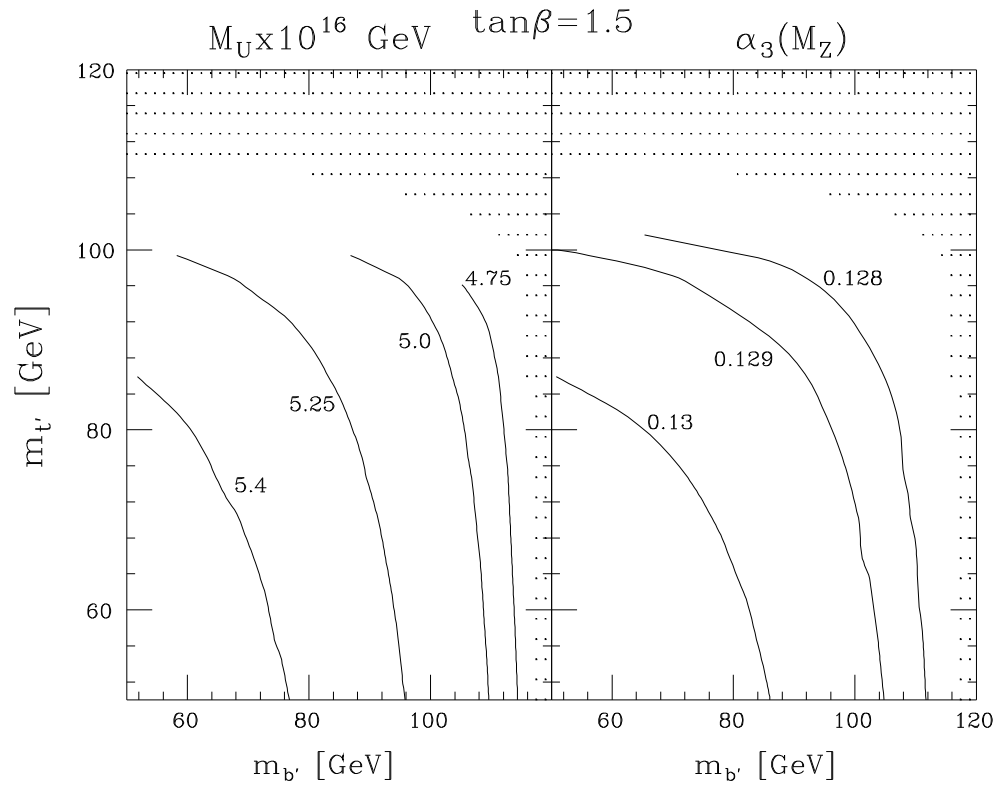

Fig. 3

Yukawas at $\mathrm{M}_{\mathrm{U}}: \mathrm{m}_{\mathrm{t}}\left(\mathrm{m}_{\mathrm{t}}\right)=165 \mathrm{GeV}, \mathrm{m}_{\mathrm{b}}\left(\mathrm{m}_{\mathrm{b}}\right)=4.6 \mathrm{GeV}$ solid: $\lambda_{t^{\prime}}$, dashes: $\lambda_{b^{\prime}}$, dotdash: $\lambda_{T^{\prime}}$

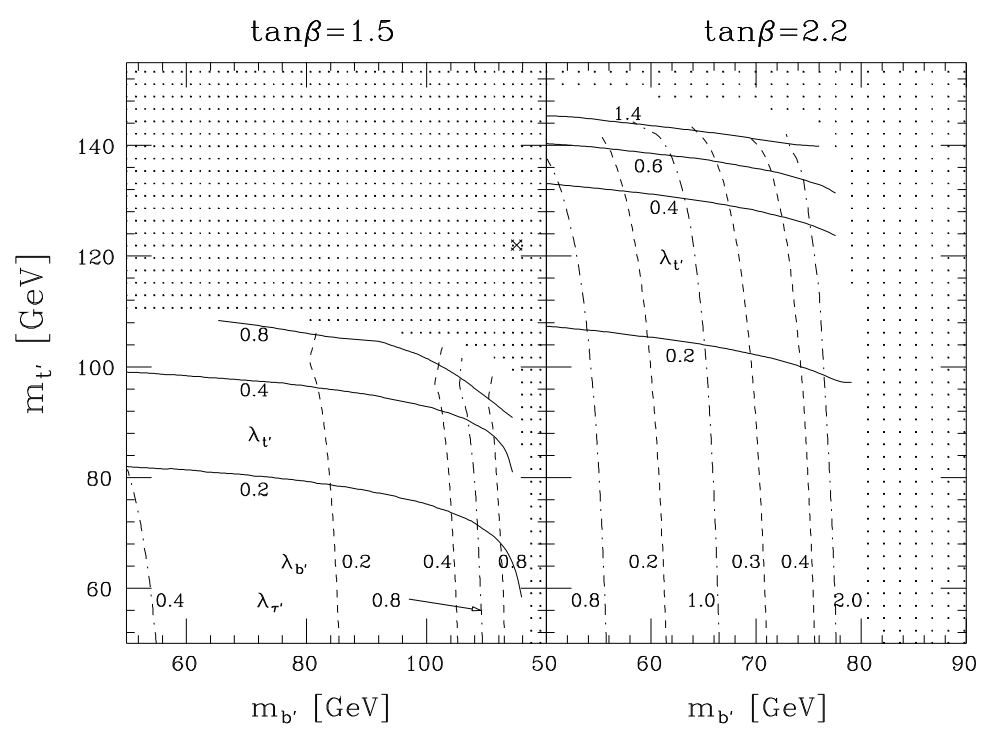

Fig. 4 


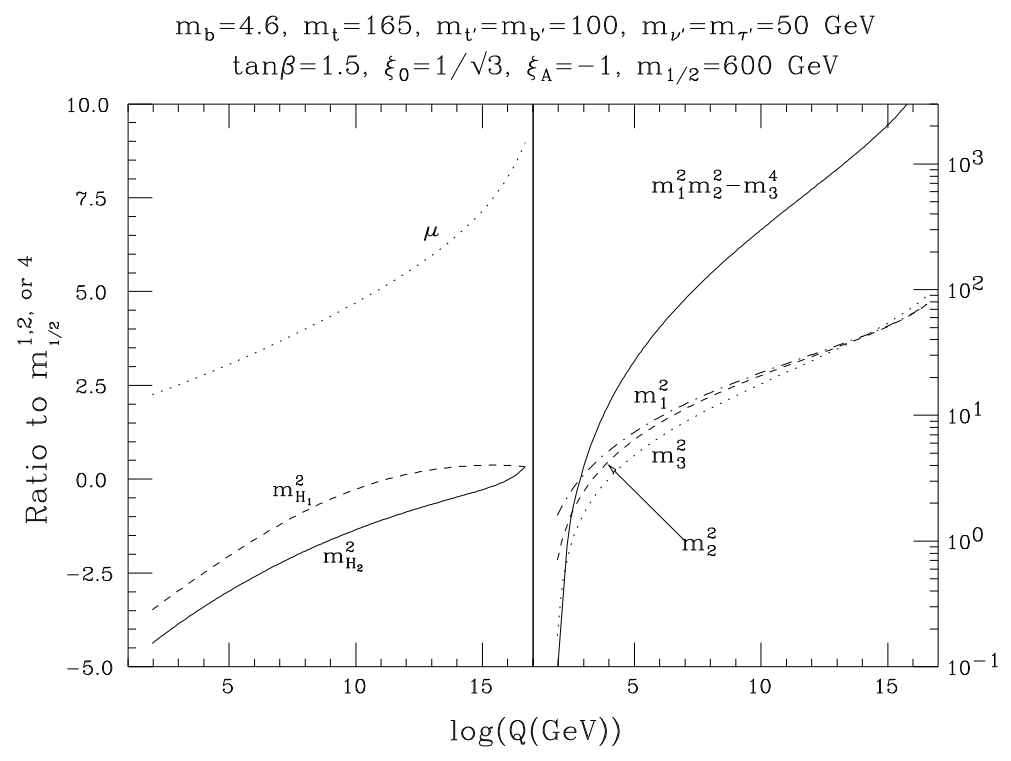

Fig. 5

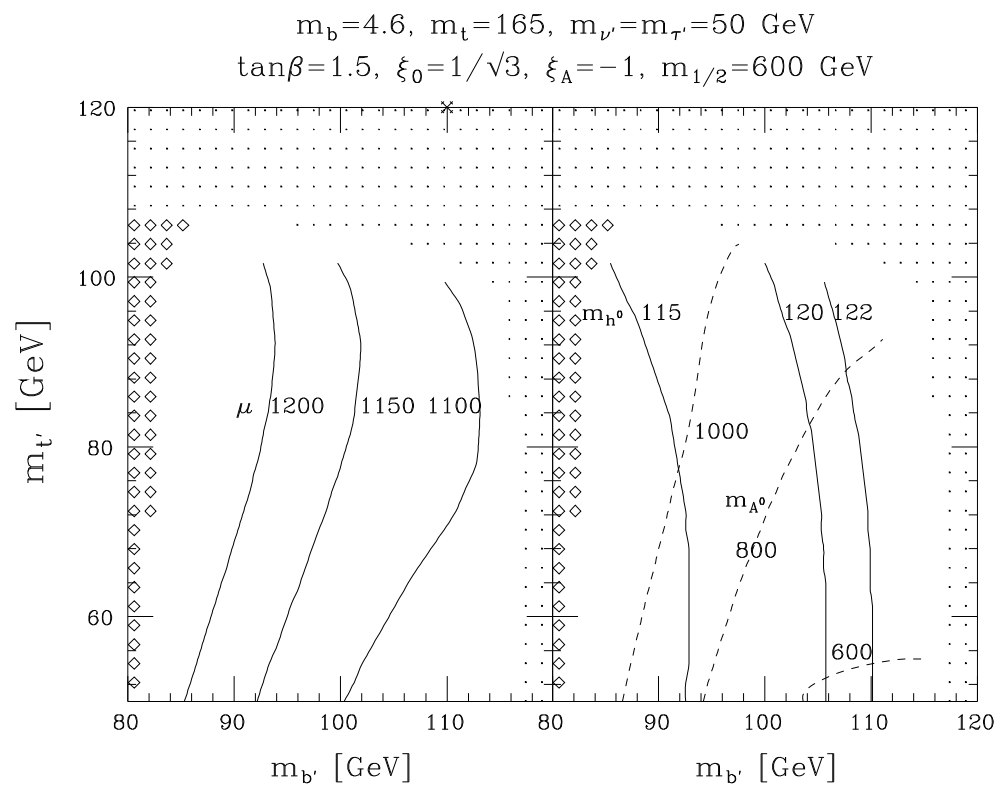

Fig. 6 
Higgs Detection at the LHC: $\mathrm{N}_{\mathrm{g}}=4$ vs. 3

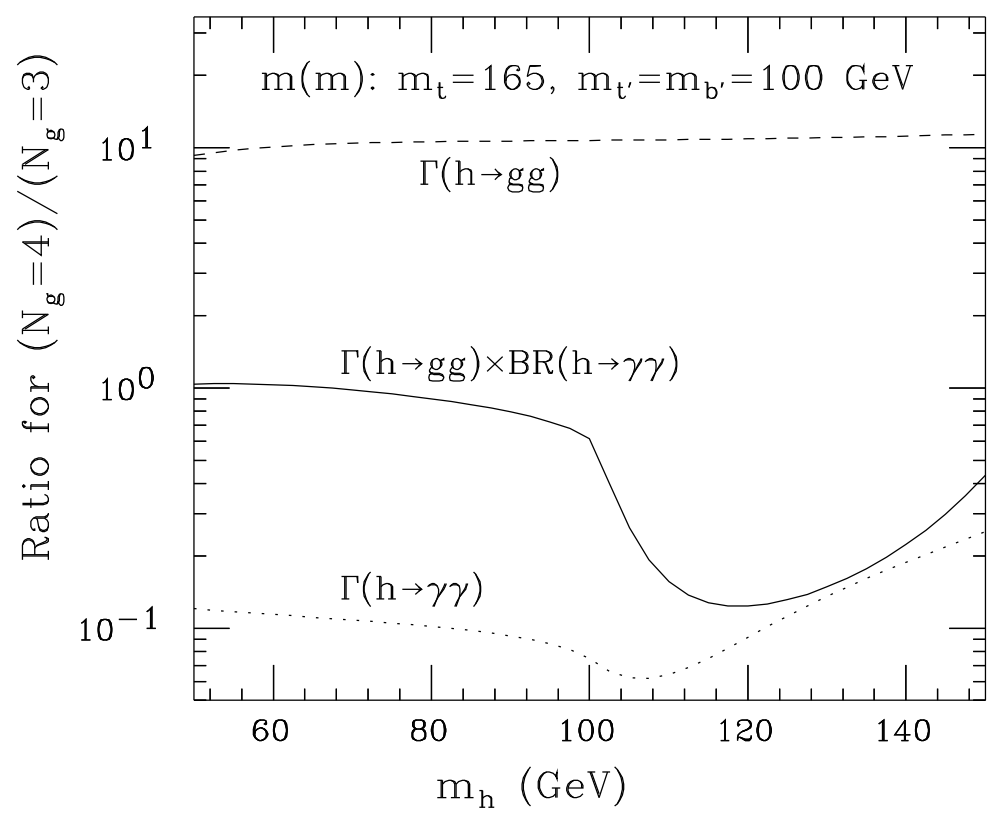

Fig. 7

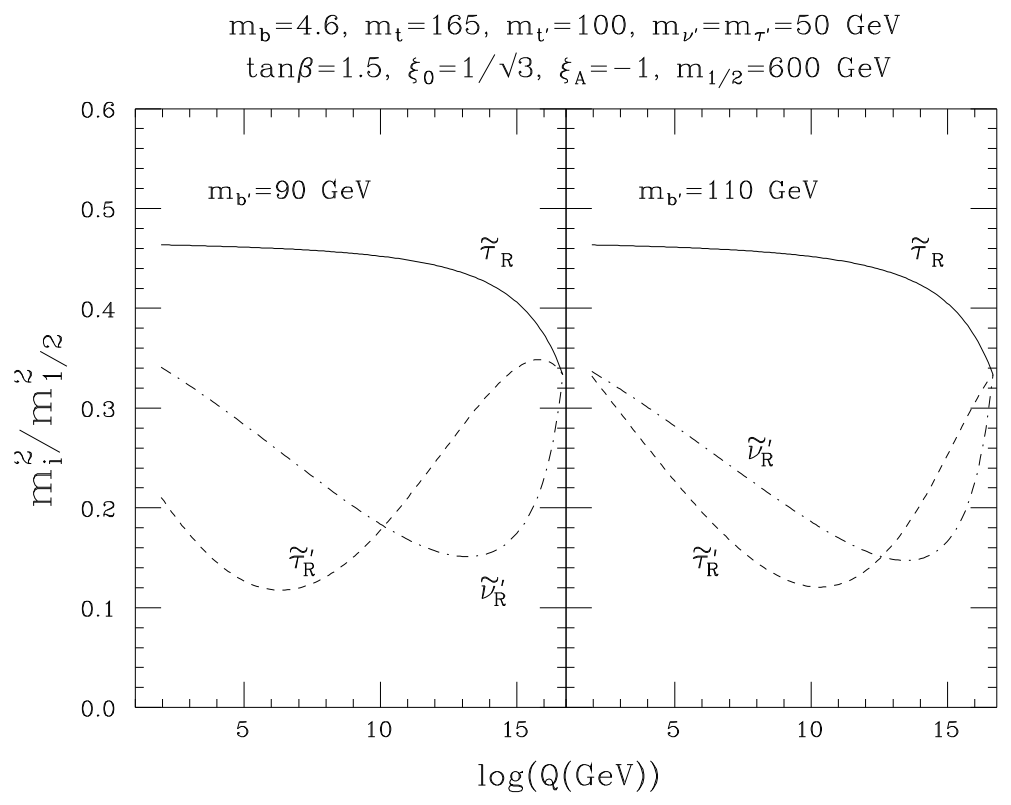

Fig. 8 


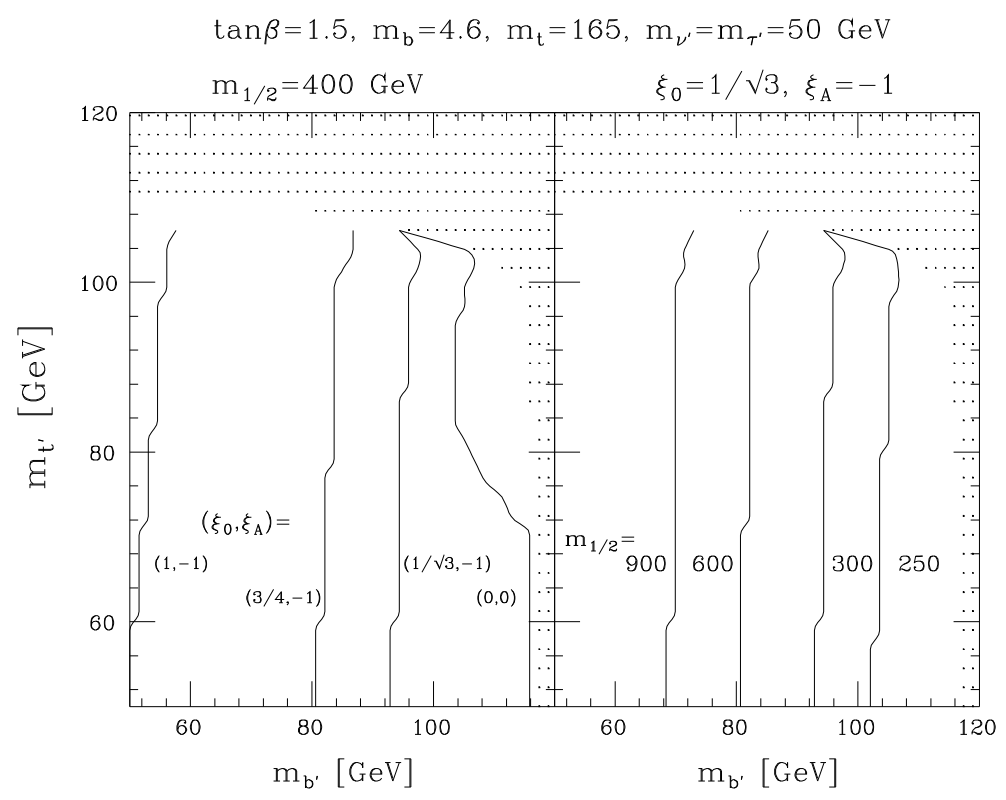

Fig. 9

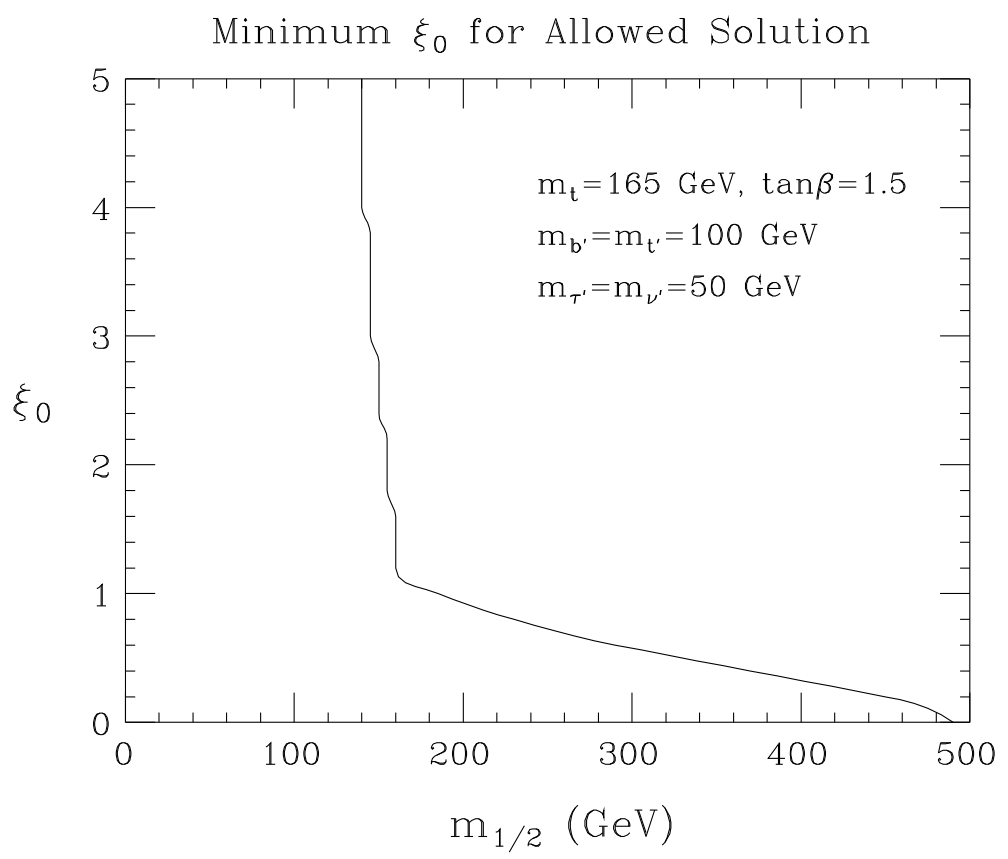

Fig. 10 
Results at Minimum Possible $\mathrm{m}_{1 / 2}$
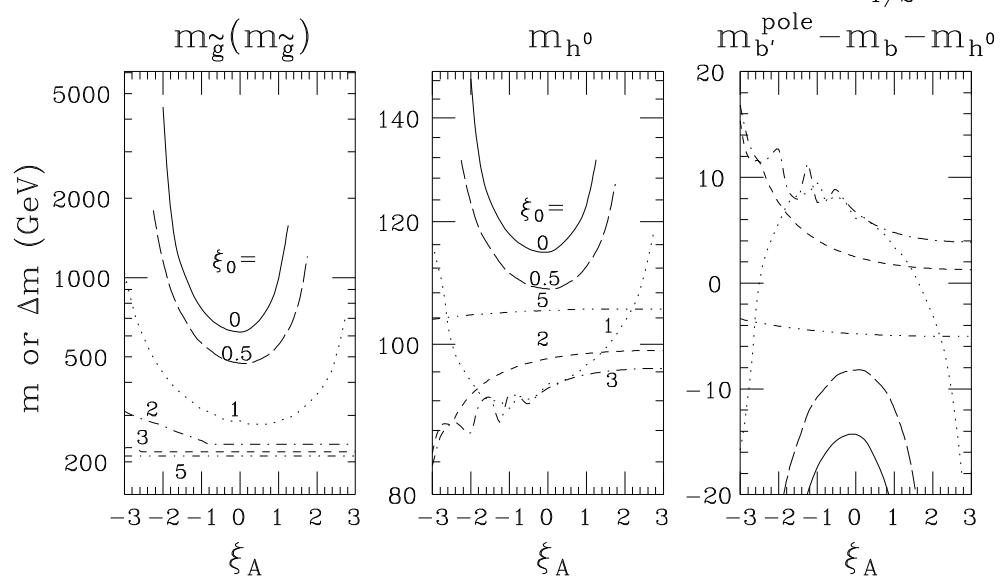

Fig. 11

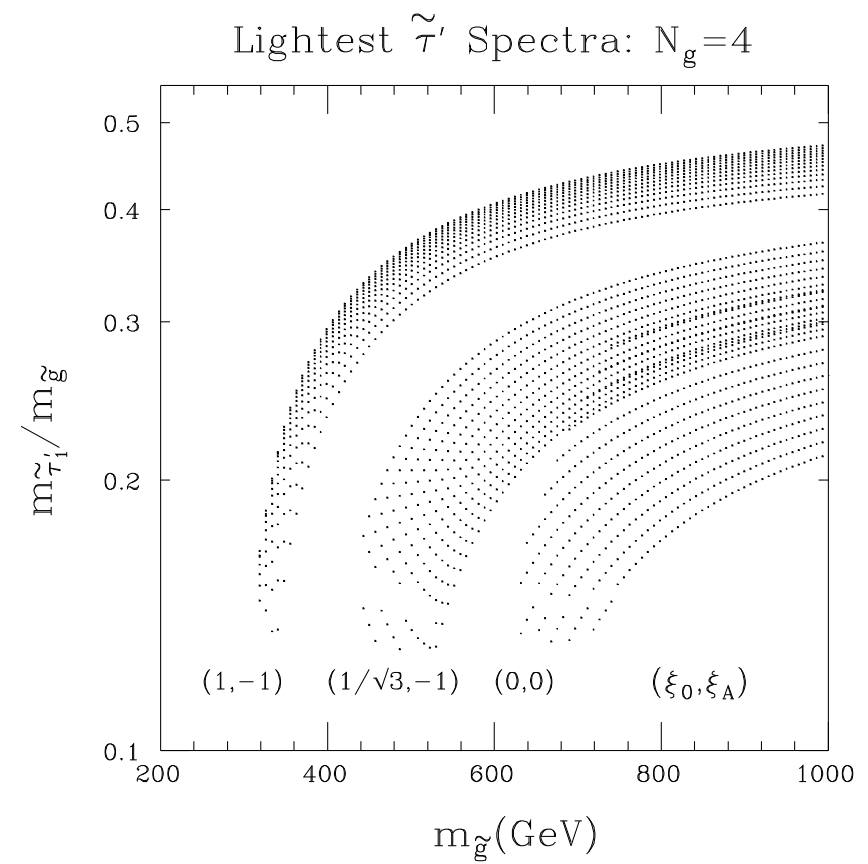

Fig. 12 


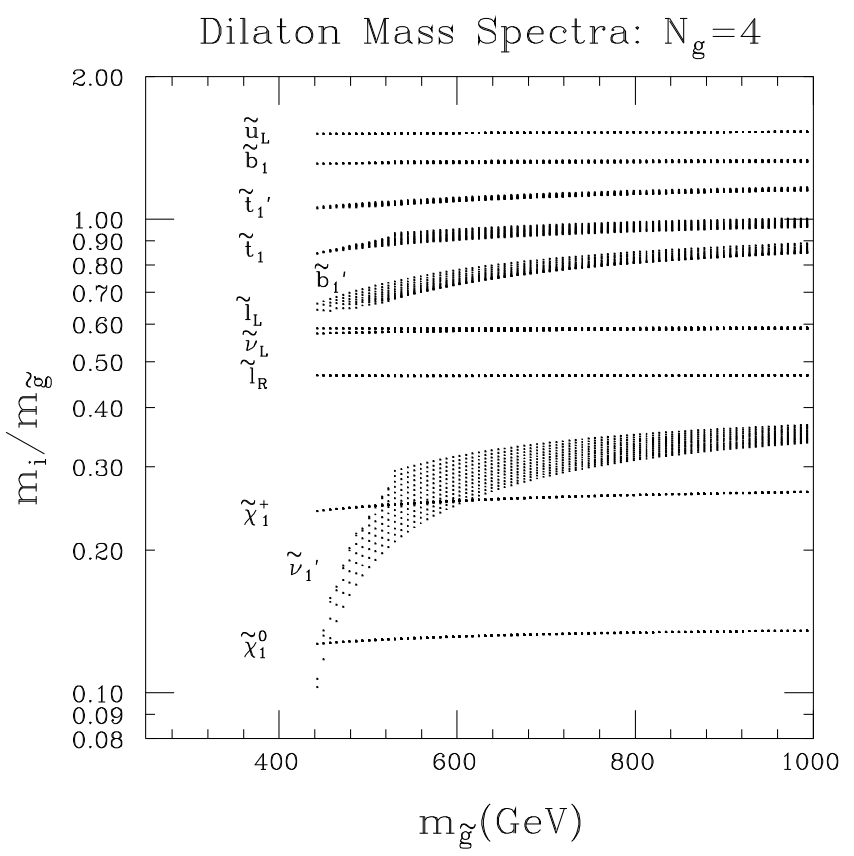

Fig. 13

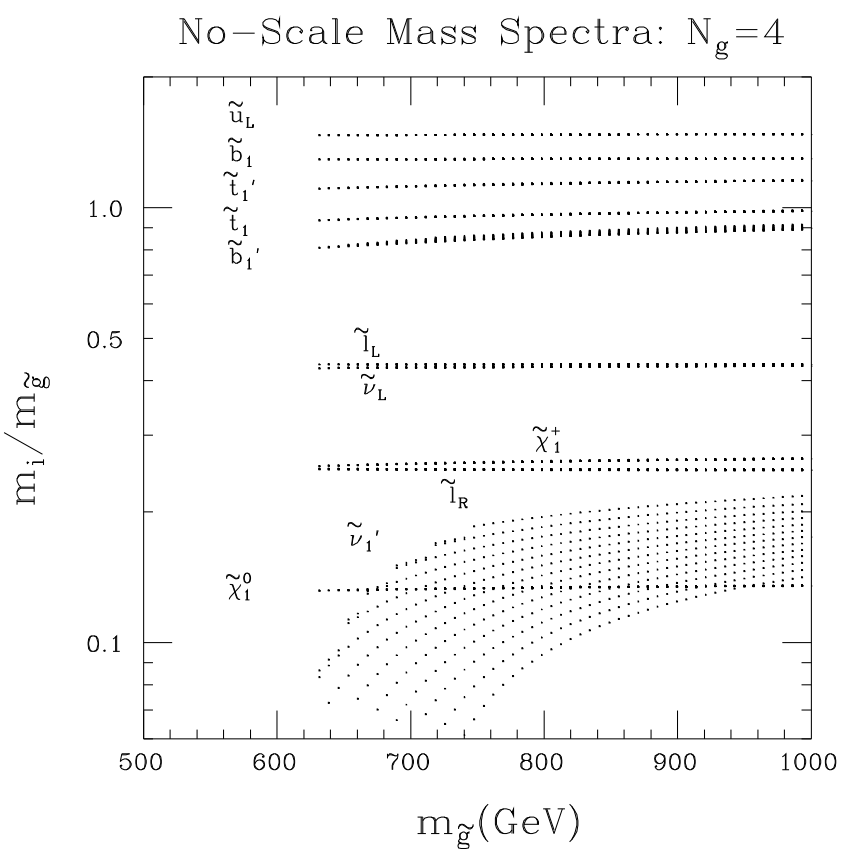

Fig. 14 


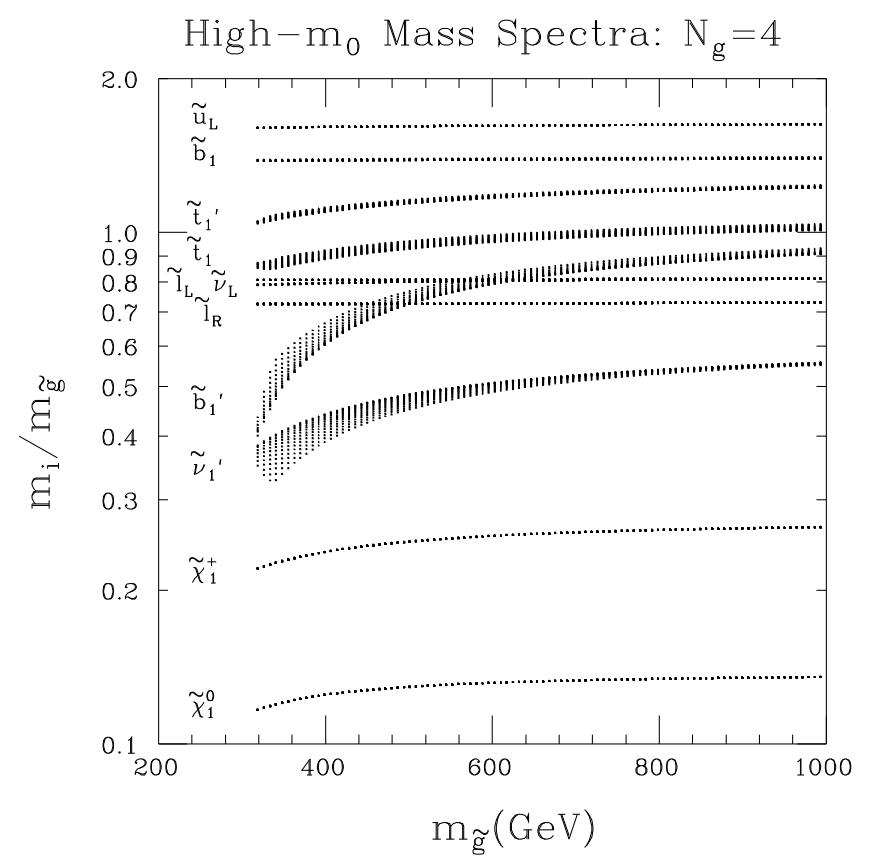

Fig. 15

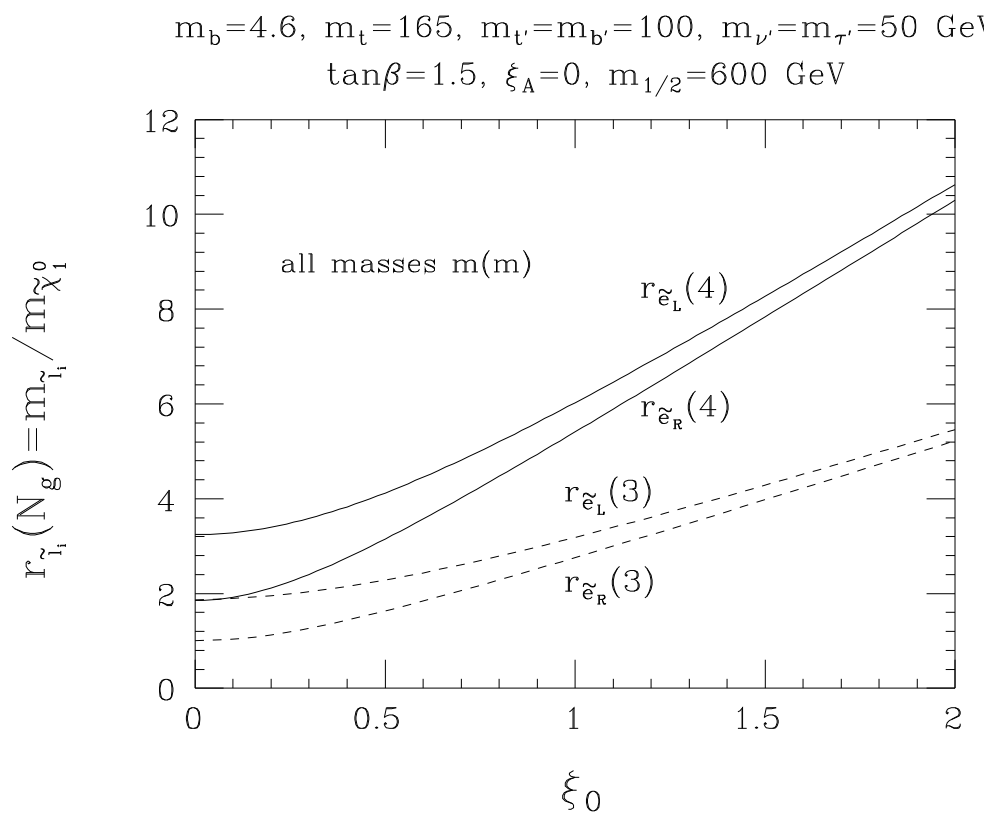

Fig. 16 


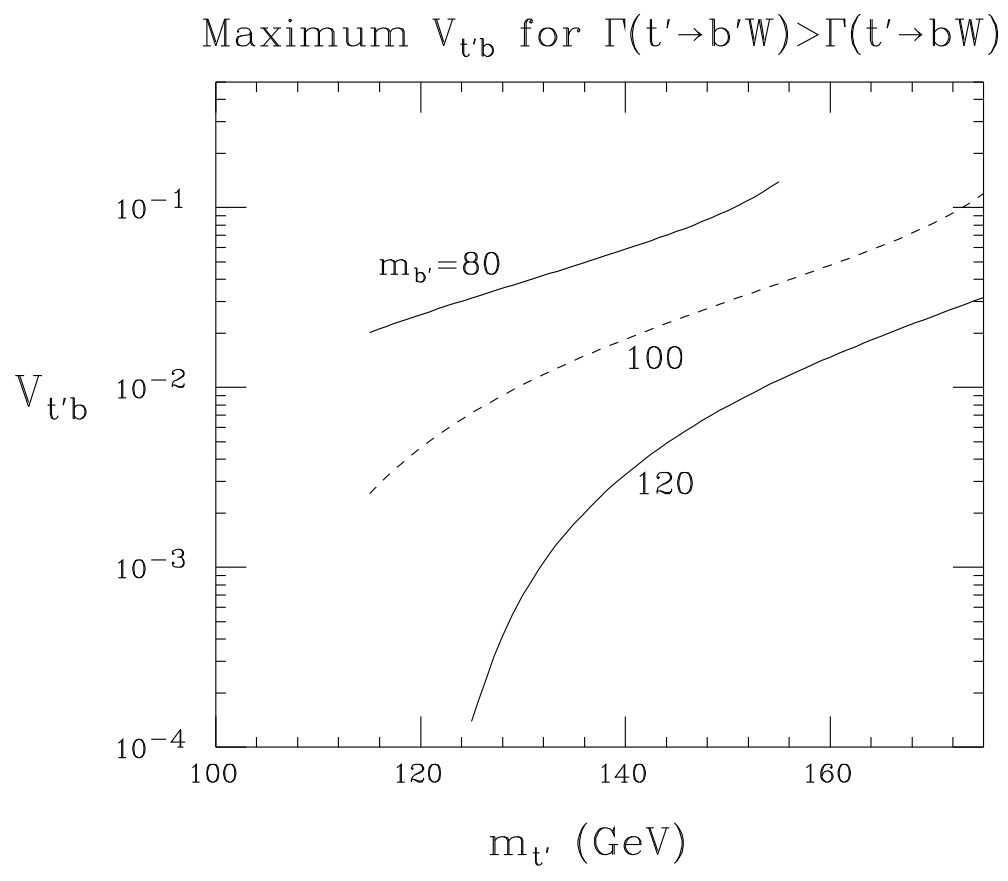

Fig. 17

Excluded by Precision EW at 90\% and 95\% CL

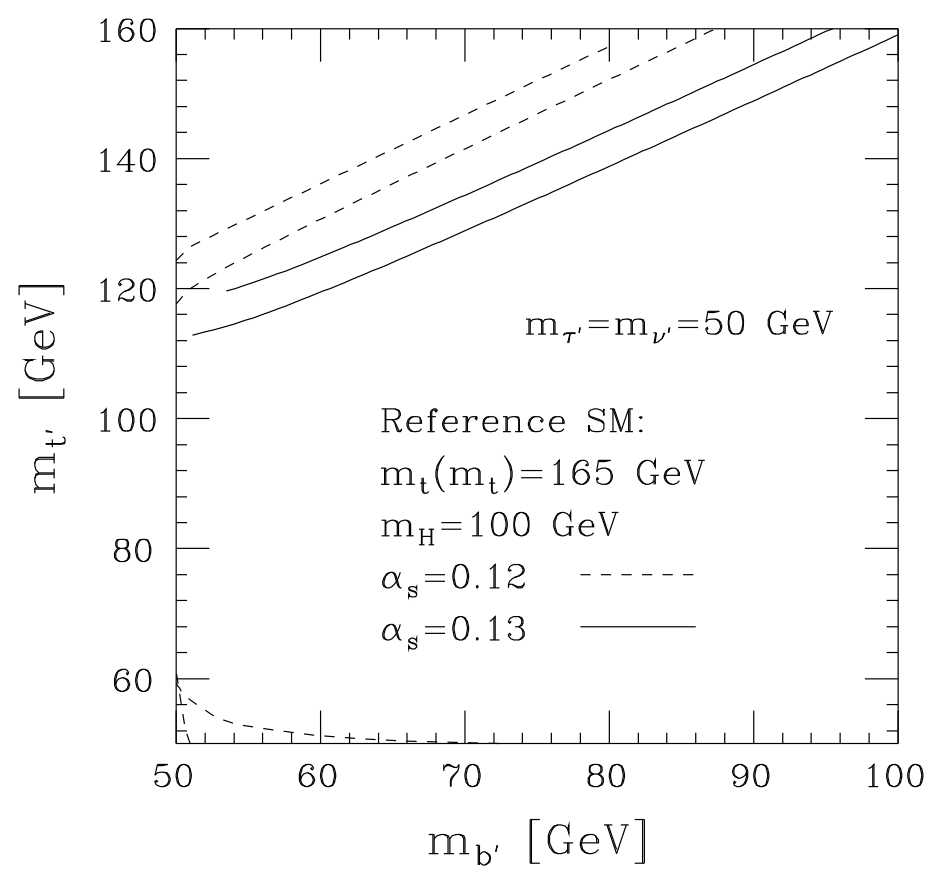

Fig. 18 


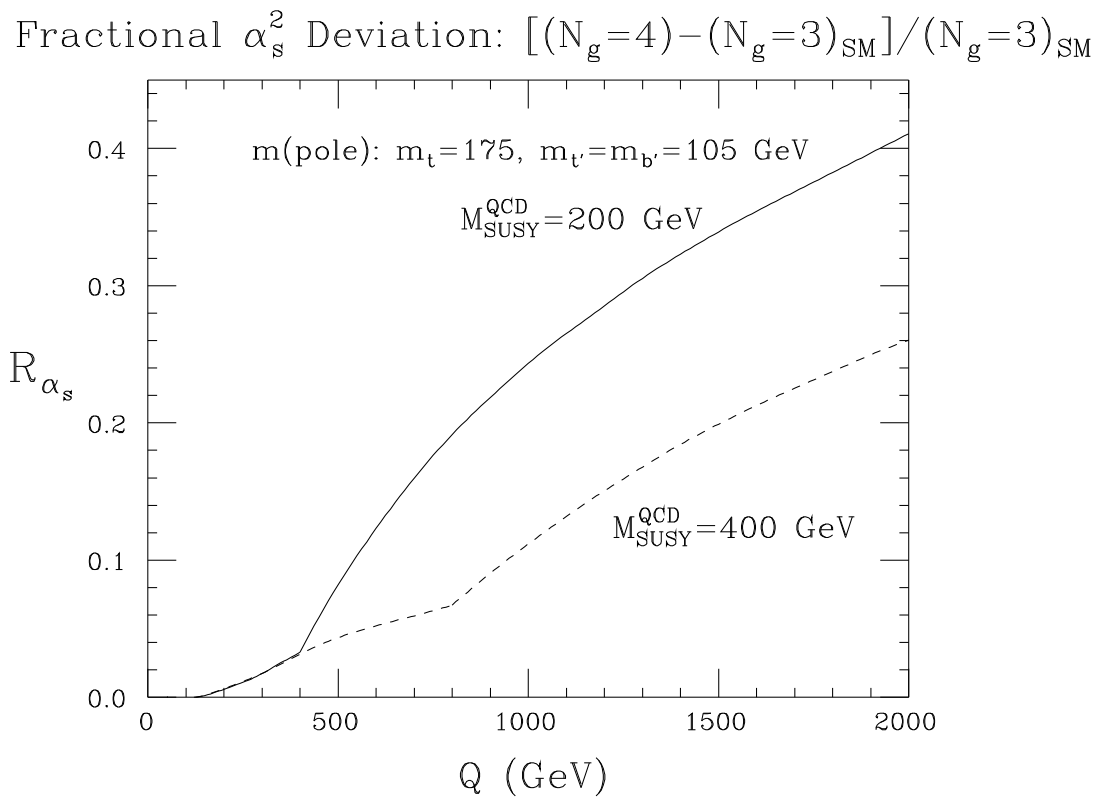

Fig. 19 